\title{
Understanding the Aggregate Effects of Credit Frictions and Uncertainty: Additional Results*
}

\author{
Nathan S. Balke ${ }^{\dagger}$ \\ Southern Methodist University and Federal Reserve Bank of Dallas \\ Enrique Martínez-García \\ Zheng Zeng§ \\ Federal Reserve Bank of Dallas \\ Bowling Green State University
}

Current Draft: June 12, 2017

\begin{abstract}
This paper integrates a financial accelerator mechanism à la Bernanke et al. (1999) and time-varying uncertainty into a medium-scale Dynamic New Keynesian model. In our model, uncertainty emerges from monetary policy (policy uncertainty) as well as from financial risks (micro uncertainty) and the aggregate state of the economy (macro uncertainty). We describe the time-variant policy, micro and macro uncertainty using a stochastic volatility model. We use this framework to identify how uncertainty propagates and its interplay with financial frictions. We also investigate how uncertainty affects the propagation of other shocks (TFP, monetary policy shocks).
\end{abstract}

JEL Classification: E32, E44, D8, C32

KEY WORDS: Stochastic Volatility, Uncertainty, Financial Accelerator, Perturbation Methods.

\footnotetext{
*An earlier draft of the paper circulated under the title "Credit Uncertainty Cycles". We acknowledge the excellent research assistance provided by Valerie Grossman. All remaining errors are ours alone. The views expressed in this paper do not necessarily reflect those of the Federal Reserve Bank of Dallas, or the Federal Reserve System.

$\dagger$ (Contacting author) Nathan S. Balke, Department of Economics, Southern Methodist University, Dallas, TX 75275. Phone: (214) 768-2693. E-mail: nbalke@smu.edu.

${ }^{\ddagger}$ Enrique Martínez-García, Federal Reserve Bank of Dallas, 2200 N. Pearl Street, Dallas, TX 75201. Phone: (214) $922-5262$. Fax: (214) 922-5194. E-mail: enrique.martinez-garcia@dal.frb.org. Webpage: https://sites.google.com/view/emgeconomics.

§Zheng Zeng, Department of Economics, Bowling Green State University, Bowling Green, OH 43403. Phone: (419) $372-8397$. E-mail: zzeng@bgsu.edu.
} 


\section{Introduction}

This paper contains a brief description of the equilibrium conditions that characterize the solution to the model used in Balke et al. (2017). The framework we employ is a medium-scale New Keynesian DSGE model with credit frictions and uncertainty (stochastic volatility). We also include an overview of the data utilized for the paper, an explanation of the codes developed for the estimation and for the quantitative simulation of the model, and a comprehensive list of the main results obtained from the model and the additional experiments conducted for robustness.

\section{Summary of Equilibrium Conditions}

\subsection{Benchmark Model}

\subsubsection{Habit Preferences}

$$
\left(C_{t}-b C_{t-1}\right)^{-\chi}-b \beta \mathbb{E}_{t}\left[\left(C_{t+1}-b C_{t}\right)^{-\chi}\right]=\Lambda_{t}
$$

where $\Lambda_{t}$ is the Lagrange multiplier on the household's budget constraint expressed in units of the final good.

\subsubsection{Goods Market}

$$
\begin{gathered}
{\left[1-\frac{\varphi_{p}}{2}\left(\Pi_{t}-1\right)^{2}\right] Y_{t}=C_{t}+X_{t}+\mu G\left(\bar{\omega}_{t-1}, \sigma_{\omega, t-1}\right) \frac{R_{t}^{e}}{\Pi_{t}} Q_{t-1} K_{t}} \\
Y_{t}=e^{\widehat{a}_{t}}\left(K_{t}\right)^{\alpha}\left(H_{t}^{e}\right)^{\vartheta}\left(H_{t}\right)^{1-\alpha-\vartheta} \\
1=\beta \mathbb{E}_{t}\left[\left(\frac{\Lambda_{t+1}}{\Lambda_{t}}\right)\left(\frac{I_{t}}{\Pi_{t+1}}\right)\right] \\
\left(1-P_{t}^{w r}\right) \epsilon=\left[1-\varphi_{p}\left(\Pi_{t}-1\right) \Pi_{t}\right]+\varphi_{p} \beta \mathbb{E}_{t}\left[\left(\frac{\Lambda_{t+1}}{\Lambda_{t}}\right)\left(\left(\Pi_{t+1}-1\right) \Pi_{t+1} \frac{Y_{t+1}}{Y_{t}}\right)\right]
\end{gathered}
$$

where the gross inflation rate on final goods is given as $\Pi_{t} \equiv \frac{P_{t}}{P_{t-1}}, \Lambda_{t}$ is the Lagrange multiplier on the household's budget constraint expressed in units of the final good, and $\widehat{a}_{t} \equiv a_{t}-a$ with $a=-\frac{1}{2} \frac{\sigma_{a}^{2}}{1-\left(\rho_{a}\right)^{2}}$.

\subsubsection{Labor Market}

$$
\begin{gathered}
W_{t}^{r}=(1-\alpha-\vartheta) \frac{P_{t}^{w r} Y_{t}}{H_{t}} \\
W_{t}^{e r}=\vartheta \frac{P_{t}^{w r} Y_{t}}{H_{t}^{e}} \\
H_{t}^{e}=1 \\
W_{t}^{r}=\frac{\kappa H_{t}^{\xi}}{\Lambda_{t}}
\end{gathered}
$$

where households' real wages are defined as $W_{t}^{r} \equiv \frac{W_{t}}{P_{t}}$ and entrepreneurial real wages as $W_{t}^{e r} \equiv \frac{W_{t}^{e}}{P_{t}}$. The relative price of the wholesale good in units of the final good is given by $P_{t}^{w r} \equiv \frac{P_{t}^{w}}{P_{t}}$. 


\subsubsection{Capital Market}

$$
\begin{aligned}
& Q_{t}=\left(\frac{\frac{X_{t}}{K_{t}}}{\delta}\right)^{\frac{1}{\varphi_{k}}} \\
& Q_{t}\left(\frac{(\delta)^{\frac{1}{\varphi_{k}}}\left(\frac{X_{t}}{K_{t}}\right)^{1-\frac{1}{\varphi_{k}}}-\frac{1}{\varphi_{k}} \delta}{1-\frac{1}{\varphi_{k}}}\right)-\frac{X_{t}}{K_{t}}-(1-\delta)\left(\bar{Q}_{t}-Q_{t}\right)=0 \\
& \frac{R_{t}^{e}}{\Pi_{t}}=\left[\frac{R_{t}^{w r}+\bar{Q}_{t}(1-\delta)}{Q_{t-1}}\right] \\
& R_{t}^{w r}=\alpha \frac{P_{t}^{w r} Y_{t}}{K_{t}} \\
& K_{t+1}=(1-\delta) K_{t}+\left(\frac{(\delta)^{\frac{1}{\varphi_{k}}}\left(\frac{X_{t}}{K_{t}}\right)^{1-\frac{1}{\varphi_{k}}}-\frac{1}{\varphi_{k}} \delta}{1-\frac{1}{\varphi_{k}}}\right) K_{t} \\
& C_{t}^{e}=(1-\gamma) \Psi\left(\bar{\omega}_{t-1}, \sigma_{\omega, t-1}\right) \frac{R_{t}^{e}}{\Pi_{t}} N_{t-1}^{r} \\
& N_{t}^{r}=W_{t}^{e r} H_{t}^{e}+\gamma \Psi\left(\bar{\omega}_{t-1}, \sigma_{\omega, t-1}\right) \frac{R_{t}^{e}}{\Pi_{t}} N_{t-1}^{r}
\end{aligned}
$$

where $R_{t}^{w r} \equiv \frac{R_{t}^{w}}{P_{t}}$ defines the real rental rate on capital in units of the final good paid by the wholesale producers to the entrepreneurs for the capital rented, and $N_{t}^{r} \equiv \frac{N_{t}}{P_{t}}$ denotes the real net worth of the entrepreneurs in units of the final good.

\subsubsection{Credit Market}

$$
\begin{aligned}
& \Phi\left(\bar{\omega}_{t} \mid \mu_{\omega, t}, \sigma_{\omega, t}\right)=\frac{1}{2}\left[1+\operatorname{erf}\left(\frac{\ln \bar{\omega}_{t}-\mu_{\omega, t}}{\sigma_{\omega} e^{\widehat{\sigma}_{\omega, t}} \sqrt[2]{2}}\right)\right] \\
& f\left(\bar{\omega}_{t}, \sigma_{\omega, t}\right)=\frac{1}{2} e^{\mu_{\omega, t}+\frac{\left(\sigma_{\omega} e^{\widehat{\sigma}_{\omega, t}}\right)^{2}}{2}}\left[1+\operatorname{erf}\left(\frac{\mu_{\omega, t}+\left(\sigma_{\omega} e^{\widehat{\sigma}_{\omega, t}}\right)^{2}-\ln \bar{\omega}_{t}}{\sigma_{\omega} e^{\widehat{\sigma}_{\omega, t}} \sqrt[2]{2}}\right)\right]-\bar{\omega}_{t}\left(1-\Phi\left(\bar{\omega}_{t} \mid \sigma_{\omega, t}\right)\right) \\
& G\left(\bar{\omega}_{t}, \sigma_{\omega, t}\right)=1-\frac{1}{2} e^{\mu_{\omega, t}+\frac{\left(\sigma_{\omega} e^{\hat{\sigma}_{\omega, t}}\right)^{2}}{2}}\left[1+\operatorname{erf}\left(\frac{\mu_{\omega, t}+\left(\sigma_{\omega} e^{\widehat{\sigma}_{\omega, t}}\right)^{2}-\ln \bar{\omega}_{t}}{\sigma_{\omega} e^{\widehat{\sigma}_{\omega, t}} \sqrt[2]{2}}\right)\right] \\
& f\left(\bar{\omega}_{t}, \sigma_{\omega, t}\right)+g\left(\bar{\omega}_{t}, \sigma_{\omega, t}\right)=1-\mu G\left(\bar{\omega}_{t}, \sigma_{\omega, t}\right) \\
& \lambda\left(\bar{\omega}_{t}, \sigma_{\omega, t}\right)\left(f_{\omega}\left(\bar{\omega}_{t}, \sigma_{\omega, t}\right)+\mu G_{\omega}\left(\bar{\omega}_{t}, \sigma_{\omega, t}\right)\right)=f_{\omega}\left(\bar{\omega}_{t}, \sigma_{\omega, t}\right) \\
& f_{\omega}\left(\bar{\omega}_{t}, \sigma_{\omega, t}\right)=-\frac{1}{2}\left[1+\operatorname{erf}\left(\frac{\mu_{\omega, t}-\ln \left(\bar{\omega}_{t}\right)}{\sigma_{\omega} e^{\widehat{\sigma}_{\omega, t}} \sqrt[2]{2}}\right)\right] \\
& G_{\omega}\left(\bar{\omega}_{t}, \sigma_{\omega, t}\right)=0.398942\left(\frac{\left(\bar{\omega}_{t}\right)^{\frac{\mu_{\omega, t}}{\left(\sigma_{\omega} e^{\tilde{\sigma}_{\omega}, t}\right)^{2}}}}{\sigma_{\omega} e^{\widehat{\sigma}_{\omega, t}}}\right) e^{-\left(\frac{\left(\mu_{\omega, t}\right)^{2}+\left(\ln \left(\overline{\bar{\omega}}_{t}\right)\right)^{2}}{2\left(\sigma_{\omega} \tilde{e}^{\tilde{\sigma}_{\omega, t}}\right)^{2}}\right)} \\
& \Psi\left(\bar{\omega}_{t}, \sigma_{\omega, t}\right)=f\left(\bar{\omega}_{t}, \sigma_{\omega, t}\right)+\lambda\left(\bar{\omega}_{t}, \sigma_{\omega, t}\right) g\left(\bar{\omega}_{t}, \sigma_{\omega, t}\right)
\end{aligned}
$$




$$
\begin{aligned}
\frac{Q_{t} K_{t+1}}{N_{t}^{r}} & =\frac{\Psi\left(\bar{\omega}_{t}, \sigma_{\omega, t}\right)}{f\left(\bar{\omega}_{t}, \sigma_{\omega, t}\right)} \\
\mathbb{E}_{t}\left(R_{t+1}^{e}\right) & =\frac{\lambda\left(\bar{\omega}_{t}, \sigma_{\omega, t}\right)}{\Psi\left(\bar{\omega}_{t}, \sigma_{\omega, t}\right)} I_{t}
\end{aligned}
$$

where the stochastic volatility of the idiosyncratic technology shock $\sigma_{\omega, t}$ is computed as $\sigma_{\omega, t}=\sigma_{\omega} e^{\widehat{\sigma}_{\omega, t}}$.

\subsubsection{Monetary Policy Rule}

$$
\frac{I_{t}}{I}=\left(\frac{I_{t-1}}{I}\right)^{\rho_{i}}\left(\left(\frac{\Pi_{t}}{\Pi_{t}^{*}}\right)^{\psi_{\pi}}\left(\frac{Y_{t}}{Y_{t-1}}\right)^{\psi_{x}}\right)^{1-\rho_{i}} e^{\widehat{m}_{t}}
$$

where $\widehat{m}_{t} \equiv m_{t}-m$ and $m=-\frac{1}{2} \frac{\sigma_{m}^{2}}{1-\left(\rho_{m}\right)^{2}}$. We also impose that the monetary authority targets zero net-inflation, i.e. $\Pi_{t}^{*}=1$.

\subsection{Exogenous Shocks with Stochastic Volatility}

Denoting $\widehat{\sigma}_{a, t} \equiv \ln \sigma_{a, t}-\ln \sigma_{a}, \widehat{\sigma}_{\omega, t} \equiv \ln \sigma_{\omega, t}-\ln \sigma_{\omega}$ and $\widehat{\sigma}_{m, t} \equiv \ln \sigma_{m, t}-\ln \sigma_{m}$, we define the shock processes as follows:

\section{Aggregate Productivity (TFP) Shock (with Macro-Uncertainty)}

$$
\begin{gathered}
\mu_{a, t}=-\frac{\left(\sigma_{a} e^{\widehat{\sigma}_{a, t}}\right)^{2}}{2}+\left(\rho_{a}\right)^{2} \mu_{a, t-1} \\
a_{t}=\mu_{a, t}+\rho_{a}\left(a_{t-1}-\mu_{a, t-1}\right)+\sigma_{a} e^{\widehat{\sigma}_{a, t}} \varepsilon_{a, t} \\
\widehat{\sigma}_{a, t}=v_{a} \widehat{\sigma}_{a, t-1}+\eta_{a} u_{a, t} \\
A_{t} \equiv e^{\widehat{a}_{t}} \text { (auxiliary equation) }
\end{gathered}
$$

where the stochastic volatility of the TFP shock $\sigma_{a, t}$ is computed as $\sigma_{a, t}=\sigma_{a} e^{\widehat{\sigma}_{a, t}}$.

\section{Micro-Uncertainty Shock}

$$
\begin{gathered}
\mu_{\omega, t}=-\frac{\left(\sigma_{\omega} e^{\widehat{\sigma}_{\omega, t}}\right)^{2}}{2} \\
\widehat{\sigma}_{\omega, t}=v_{\omega} \widehat{\sigma}_{\omega, t-1}+\eta_{\omega} u_{\omega, t} \\
O_{t} \equiv \mathbb{E}\left(\omega_{t}\right)=e^{\mu_{\omega, t}+\frac{\left(\sigma_{\omega} e^{\hat{\sigma}_{\omega, t}}\right)^{2}}{2}} \text { (auxiliary equation) }
\end{gathered}
$$

where the stochastic volatility of the idiosyncratic technology shock $\sigma_{\omega, t}$ is computed as $\sigma_{\omega, t}=\sigma_{\omega} e^{\widehat{\sigma}_{\omega, t}}$.

Monetary Policy Shock (with Policy-Uncertainty)

$$
\begin{gathered}
\mu_{m, t}=-\frac{\left(\sigma_{m} e^{\widehat{\sigma}_{m, t}}\right)^{2}}{2}+\left(\rho_{m}\right)^{2} \mu_{m, t-1} \\
m_{t}=\mu_{m, t}+\rho_{m}\left(m_{t-1}-\mu_{m, t-1}\right)+\sigma_{m} e^{\widehat{\sigma}_{m, t}} \varepsilon_{m, t}
\end{gathered}
$$




$$
\begin{gathered}
\widehat{\sigma}_{m, t}=v_{m} \widehat{\sigma}_{m, t-1}+\eta_{m} u_{m, t} \\
M_{t} \simeq 1+\widehat{m}_{t} \text { (auxiliary equation) }
\end{gathered}
$$

where the stochastic volatility of the monetary policy shock $\sigma_{m, t}$ is computed as $\sigma_{m, t}=\sigma_{m} e^{\widehat{\sigma}_{m, t}}$.

\section{Deterministic Steady State}

\subsection{Habit Preferences}

$$
(1-b \beta)(1-b)^{-\chi} C^{-\chi}=\Lambda
$$

\subsection{Goods Market}

$$
\begin{gathered}
{\left[1-\frac{\varphi_{p}}{2}(\Pi-1)^{2}\right] Y=C+X+\mu G\left(\bar{\omega}, \sigma_{\omega}\right) \frac{R^{e}}{\Pi} Q K} \\
Y=e^{\widehat{a}}(K)^{\alpha}\left(H^{e}\right)^{\vartheta}(H)^{1-\alpha-\vartheta} \\
1=\beta\left(\frac{I}{\Pi}\right) \\
\left(1-P^{w r}\right) \epsilon=1-(1-\beta) \varphi_{p}(\Pi-1) \Pi
\end{gathered}
$$

where $\widehat{a}=0$.

\subsection{Labor Market}

$$
\begin{gathered}
W^{r}=(1-\alpha-\vartheta) \frac{P^{w r} Y}{H} \\
W^{e r}=\vartheta \frac{P^{w r} Y}{H^{e}} \\
H^{e}=1 \\
W^{r}=\frac{\kappa H^{\xi}}{\Lambda}
\end{gathered}
$$

\subsection{Capital Market}

$$
\begin{gathered}
Q=\left(\frac{\frac{X}{K}}{\delta}\right)^{\frac{1}{\varphi_{k}}} \\
Q\left(\frac{(\delta)^{\frac{1}{\varphi_{k}}}\left(\frac{X}{K}\right)^{1-\frac{1}{\varphi_{k}}}-\frac{1}{\varphi_{k}} \delta}{1-\frac{1}{\varphi_{k}}}\right)-\frac{X}{K}-(1-\delta)(\bar{Q}-Q)=0 \\
\frac{R^{e}}{\Pi}=\left[\frac{R^{w r}+\bar{Q}(1-\delta)}{Q}\right] \\
R^{w r}=\alpha \frac{P^{w r} Y}{K}
\end{gathered}
$$




$$
\begin{gathered}
K=(1-\delta) K+\left(\frac{(\delta)^{\frac{1}{\varphi_{k}}}\left(\frac{X}{K}\right)^{1-\frac{1}{\varphi_{k}}}-\frac{1}{\varphi_{k}} \delta}{1-\frac{1}{\varphi_{k}}}\right) K \\
C^{e}=(1-\gamma) \Psi\left(\bar{\omega}, \sigma_{\omega}\right) \frac{R^{e}}{\Pi} N^{r} \\
N^{r}=W^{e r} H^{e}+\gamma \Psi\left(\bar{\omega}, \sigma_{\omega}\right) \frac{R^{e}}{\Pi} N^{r}
\end{gathered}
$$

\subsection{Credit Market}

$$
\begin{gathered}
\Phi\left(\bar{\omega} \mid \mu_{\omega}, \sigma_{\omega}\right)=\frac{1}{2}\left[1+\operatorname{erf}\left(\frac{\ln \bar{\omega}-\mu_{\omega}}{\sigma_{\omega} e^{\widehat{\sigma}_{\omega}} \sqrt[2]{2}}\right)\right] \\
f\left(\bar{\omega}, \sigma_{\omega}\right)=\frac{1}{2} e^{\mu_{\omega}+\frac{\left(\sigma_{\omega} e^{\hat{\sigma}_{\omega}}\right)^{2}}{2}}\left[1+\operatorname{erf}\left(\frac{\mu_{\omega}+\left(\sigma_{\omega} e^{\widehat{\sigma}_{\omega}}\right)^{2}-\ln \bar{\omega}}{\sigma_{\omega} e^{\widehat{\sigma}_{\omega}} \sqrt[2]{2}}\right)\right]-\bar{\omega}\left(1-\Phi\left(\bar{\omega} \mid \sigma_{\omega}\right)\right) \\
G\left(\bar{\omega}, \sigma_{\omega}\right)=1-\frac{1}{2} e^{\mu_{\omega}+\frac{\left(\sigma_{\omega} e^{\widehat{\sigma}_{\omega}}\right)^{2}}{2}}\left[1+\operatorname{erf}\left(\frac{\mu_{\omega}+\left(\sigma_{\omega} e^{\widehat{\sigma}_{\omega}}\right)^{2}-\ln \bar{\omega}}{\sigma_{\omega} e^{\widehat{\sigma}_{\omega}} \sqrt[2]{2}}\right)\right] \\
f\left(\bar{\omega}, \sigma_{\omega}\right)+g\left(\bar{\omega}, \sigma_{\omega}\right)=1-\mu G\left(\bar{\omega}, \sigma_{\omega}\right) \\
\lambda\left(\bar{\omega}, \sigma_{\omega}\right)\left(f_{\omega}\left(\bar{\omega}, \sigma_{\omega}\right)+\mu G_{\omega}\left(\bar{\omega}, \sigma_{\omega}\right)\right)=f_{\omega}\left(\bar{\omega}, \sigma_{\omega}\right) \\
f_{\omega}\left(\bar{\omega}, \sigma_{\omega}\right)=-\frac{1}{2}\left[1+\operatorname{erf}\left(\frac{\mu_{\omega}-\ln (\bar{\omega})}{\sigma_{\omega} \sqrt[2]{2}}\right)\right] \\
G_{\omega}\left(\bar{\omega}, \sigma_{\omega}\right)=0.398942\left(\frac{(\bar{\omega})}{\left(\sigma_{\omega} e^{\widehat{\sigma}_{\omega}}\right)}\right) e^{-\left(\frac{\left(\mu_{\omega}\right)^{2}+(\ln (\bar{\omega}))^{2}}{2\left(\sigma_{\omega} e^{\widehat{\sigma}_{\omega}}\right)^{2}}\right)} \\
\Psi\left(\bar{\omega}, \sigma_{\omega}\right)=f\left(\bar{\omega}, \sigma_{\omega}\right)+\lambda\left(\bar{\omega}, \sigma_{\omega}\right) g\left(\bar{\omega}, \sigma_{\omega}\right) \\
\frac{Q K}{N^{r}}=\frac{\Psi\left(\bar{\omega}, \sigma_{\omega}\right)}{f\left(\bar{\omega}, \sigma_{\omega}\right)} \\
R^{e}=\frac{\lambda\left(\bar{\omega}, \sigma_{\omega}\right)}{\Psi\left(\bar{\omega}, \sigma_{\omega}\right)} I
\end{gathered}
$$

\subsection{Monetary Policy Rule}

$$
\frac{I}{I}=\left(\frac{I}{I}\right)^{\rho_{i}}\left(\left(\frac{\Pi}{\Pi^{*}}\right)^{\psi_{\pi}}\left(\frac{Y}{Y}\right)^{\psi_{x}}\right)^{1-\rho_{i}} e^{\widehat{m}}
$$

where $\widehat{m}=0$.

\subsection{Exogenous Shocks with Stochastic Volatility}

Aggregate Productivity (TFP) Shock (with Macro-Uncertainty)

$$
\mu_{a}=-\frac{\left(\sigma_{a} e^{\widehat{\sigma}_{a}}\right)^{2}}{2}+\left(\rho_{a}\right)^{2} \mu_{a}
$$




$$
\begin{gathered}
a=\mu_{a}+\rho_{a}\left(a-\mu_{a}\right)+\sigma_{a} e^{\widehat{\sigma}_{a}} \cdot 0 \\
\widehat{\sigma}_{a}=v_{a} \widehat{\sigma}_{a}+\eta_{a} \cdot 0 \\
A \equiv e^{\widehat{a}} \text { (auxiliary equation) }
\end{gathered}
$$

Micro-Uncertainty Shock

$$
\begin{gathered}
\mu_{\omega}=-\frac{\left(\sigma_{\omega} e^{\widehat{\sigma}_{\omega}}\right)^{2}}{2} \\
\widehat{\sigma}_{\omega}=v_{\omega} \widehat{\sigma}_{\omega}+\eta_{\omega} \cdot 0 \\
O_{t} \equiv e^{\mu_{\omega}+\frac{\left(\sigma_{\omega} e^{\widehat{\sigma}_{\omega}}\right)^{2}}{2}} \text { (auxiliary equation) }
\end{gathered}
$$

Monetary Policy Shock (with Policy-Uncertainty)

$$
\begin{gathered}
\mu_{m}=-\frac{\left(\sigma_{m} e^{\widehat{\sigma}_{m}}\right)^{2}}{2}+\left(\rho_{m}\right)^{2} \mu_{m} \\
m=\mu_{m}+\rho_{m}\left(m-\mu_{m}\right)+\sigma_{m} e^{\widehat{\sigma}_{m}} \cdot 0 \\
\widehat{\sigma}_{m}=v_{m} \widehat{\sigma}_{m}+\eta_{m} \cdot 0 \\
M \simeq 1+\widehat{m} \text { (auxiliary equation) }
\end{gathered}
$$

\subsection{Steady State and Structural Parameters}

The zero-inflation deterministic steady state of the model can be specified as follows:

$$
\Pi=\Pi^{*}=1
$$

Let us impose $\widehat{a}=\widehat{m}=0$ and let's ignore the exogenous show equations in (66) - (76) for now. This ensures that the monetary policy rule in (65) is satisfied in steady state. We also assume that the characterization of the steady state implies that,

$$
\begin{aligned}
& X=\delta K, \\
& Q=1 .
\end{aligned}
$$

This satisfies (48) and (52) trivially, but it also implies from (49) that,

$$
\bar{Q}=1
$$

From (43), it follows that,

$$
P^{w r}=\frac{\epsilon-1}{\epsilon}
$$

and from (42) we get that,

$$
I=\frac{1}{\beta},
$$


while (46) simply says,

$$
H^{e}=1
$$

Then, we can re-write (39) in the following terms,

$$
\Lambda=(1-b \beta)(1-b)^{-\chi} C^{-\chi}
$$

All these steady state conditions hold in equilibrium and are straightforward to characterize in setting up the deterministic steady state for the model.

The deterministic steady state of the different shock processes described in (66) - (76) can be summarized now as follows,

$$
\begin{aligned}
\mu_{a} & =-\frac{1}{2} \frac{\sigma_{a}^{2}}{1-\left(\rho_{a}\right)^{2}}, \\
a & =\mu_{a}, \\
\widehat{\sigma}_{a} & =0, \\
A & \equiv 1 \Leftrightarrow \widehat{a}=0,
\end{aligned}
$$

and

$$
\begin{aligned}
\mu_{\omega} & =-\frac{\sigma_{\omega}^{2}}{2}, \\
\widehat{\sigma}_{\omega} & =0, \\
O_{t} & =1,
\end{aligned}
$$

and

$$
\begin{aligned}
\mu_{m} & =-\frac{1}{2} \frac{\sigma_{m}^{2}}{1-\left(\rho_{m}\right)^{2}}, \\
m & =\mu_{m}, \\
\widehat{\sigma}_{m} & =0, \\
M & \equiv 1 \Leftrightarrow \widehat{m}=0 .
\end{aligned}
$$

Finally, the reminder of the steady state conditions not related to the loan contract-i.e., (40), (41), (44), (45), (47), (50), (51), (53) and (54) — can be re-written as,

$$
\begin{aligned}
& Y=C+\delta K+\mu G\left(\bar{\omega}, \sigma_{\omega}\right) R^{e} K, \\
& Y=e^{\widehat{a}}(K)^{\alpha}(H)^{1-\alpha-\vartheta},
\end{aligned}
$$




$$
\begin{aligned}
W^{r} & =(1-\alpha-\vartheta)\left(\frac{\epsilon-1}{\epsilon}\right) \frac{Y}{H}, \\
W^{e r} & =\vartheta\left(\frac{\epsilon-1}{\epsilon}\right) Y, \\
W^{r} & =\left(\frac{(1-b)^{\chi}}{1-b \beta}\right) \kappa H^{\xi} C^{\chi}, \\
R^{e} & =R^{w r}+(1-\delta), \\
R^{w r} & =\alpha\left(\frac{\epsilon-1}{\epsilon}\right) \frac{Y}{K}, \\
C^{e} & =(1-\gamma) \Psi\left(\bar{\omega}, \sigma_{\omega}\right) R^{e} N^{r}, \\
N^{r} & =\frac{W^{e r}}{1-\gamma \Psi\left(\bar{\omega}, \sigma_{\omega}\right) R^{e}} .
\end{aligned}
$$

The deterministic steady state is then complete with the corresponding equations that characterize the terms of the loan contract under log-normality in $(55)-(64)$, i.e. with

$$
\begin{aligned}
\Phi\left(\bar{\omega} \mid \sigma_{\omega}\right) & =\frac{1}{2}\left[1+\operatorname{erf}\left(\frac{\ln \bar{\omega}+\frac{\sigma_{\omega}^{2}}{2}}{\sigma_{\omega} \sqrt[2]{2}}\right)\right] \\
f\left(\bar{\omega}, \sigma_{\omega}\right) & =\frac{1}{2}\left[1+\operatorname{erf}\left(\frac{\frac{\sigma_{\omega}^{2}}{2}-\ln \bar{\omega}}{\sigma_{\omega} \sqrt[2]{2}}\right)\right]-\bar{\omega}\left(1-\Phi\left(\bar{\omega} \mid \sigma_{\omega}\right)\right), \\
G\left(\bar{\omega}, \sigma_{\omega}\right) & =1-\frac{1}{2}\left[1+\operatorname{erf}\left(\frac{\frac{\sigma_{\omega}^{2}}{2}-\ln \bar{\omega}}{\sigma_{\omega} \sqrt[2]{2}}\right)\right] \\
\lambda\left(\bar{\omega}, \sigma_{\omega}\right)\left(f_{\omega}\left(\bar{\omega}, \sigma_{\omega}\right)+\mu \sigma_{\omega}\right)+g\left(\bar{\omega}, \sigma_{\omega}\right) & \left.=1-\mu G\left(\bar{\omega}, \sigma_{\omega}\right)\right)=f_{\omega}\left(\bar{\omega}, \sigma_{\omega}\right), \\
f_{\omega}\left(\bar{\omega}, \sigma_{\omega}\right) & =-\frac{1}{2}\left[1+\operatorname{erf}\left(\frac{-\frac{\sigma_{\omega}^{2}}{2}-\ln \bar{\omega}}{\left.\left.\sigma_{\omega} \sqrt[2]{2}\right)\right]}\right.\right. \\
G_{\omega}\left(\bar{\omega}, \sigma_{\omega}\right) & =0.398942\left(\frac{1}{\sigma_{\omega} \sqrt[2]{\bar{\omega}}}\right) e\left(\frac{\left(-\frac{\sigma_{\omega}^{2}}{2}\right)^{2}+(\ln \bar{\omega})^{2}}{2 \sigma_{\omega}^{2}}\right) \\
\Psi\left(\bar{\omega}, \sigma_{\omega}\right) & =f\left(\bar{\omega}, \sigma_{\omega}\right)+\lambda\left(\bar{\omega}, \sigma_{\omega}\right) g\left(\bar{\omega}, \sigma_{\omega}\right) \\
\frac{K}{N^{r}} & =\frac{\Psi\left(\bar{\omega}, \sigma_{\omega}\right)}{f\left(\bar{\omega}, \sigma_{\omega}\right)}, \\
R^{e} & =\frac{\lambda\left(\bar{\omega}, \sigma_{\omega}\right)}{\Psi\left(\bar{\omega}, \sigma_{\omega}\right)} \frac{1}{\beta} .
\end{aligned}
$$

We solve this subset of equations to characterize the deterministic steady state of the model with frictions using the nonlinear solver fsolve in Matlab.

Remark: On Matters of Implementation. The deterministic steady state for the model can be expressed in a somewhat more tractable manner using equation (55) - which defines the probability of default 
in steady state $\Phi\left(\bar{\omega} \mid \sigma_{\omega}\right)$ under log-normality —as follows,

$$
\begin{aligned}
\Phi\left(\bar{\omega} \mid \sigma_{\omega}\right) & =\frac{1}{2}\left[1+\operatorname{erf}\left(\frac{\ln \bar{\omega}+\frac{\sigma_{\omega}^{2}}{2}}{\sigma_{\omega} \sqrt[2]{2}}\right)\right] \\
& \Leftrightarrow \operatorname{erf}\left(\frac{\ln \bar{\omega}+\frac{\sigma_{\omega}^{2}}{2}}{\sigma_{\omega} \sqrt[2]{2}}\right)=2 \Phi\left(\bar{\omega} \mid \sigma_{\omega}\right)-1 \\
& \Leftrightarrow \operatorname{erf}^{-1}\left(\operatorname{erf}\left(\frac{\ln \bar{\omega}+\frac{\sigma_{\omega}^{2}}{2}}{\sigma_{\omega} \sqrt[2]{2}}\right)\right)=\operatorname{erf}^{-1}\left(2 \Phi\left(\bar{\omega} \mid \sigma_{\omega}\right)-1\right) .
\end{aligned}
$$

From here, we get that,

$$
\ln \bar{\omega}=\sigma_{\omega} \sqrt[2]{2}\left[\operatorname{erf}^{-1}\left(2 \Phi\left(\bar{\omega} \mid \sigma_{\omega}\right)-1\right)\right]-\frac{\sigma_{\omega}^{2}}{2}
$$

or simply

$$
\bar{\omega}=e^{\sigma_{\omega} \sqrt[2]{2}\left[\operatorname{erf}^{-1}\left(2 \Phi\left(\bar{\omega} \mid \sigma_{\omega}\right)-1\right)\right]-\frac{\sigma_{\omega}^{2}}{2}} .
$$

As a result, the steady state default threshold $\bar{\omega}$ can be expressed as a function of the steady state probability of default $\Phi\left(\bar{\omega} \mid \sigma_{\omega}\right)$. This is useful to facilitate the parameterization of the model.

\section{Data}

Through the Federal Reserve Bank of St. Louis' FRED database, we collect data on real GDP, consumption, investment and hours worked in per capita terms, together with time series for the real wage, the leverage of nonfinancial corporations, the inflation rate, the short-term nominal interest rate and the interest rate spread for the U.S. economy. The data we use is quarterly and covers the period from 1984:Q1 to 2014:Q4.

Our measure of output is the real GDP from the U.S. Bureau of Economic Analysis' (BEA) National Income and Product Accounts (NIPA) in billions of Chained 2009 Dollars. Our measure of consumption is the sum of nondurable and services consumption, also in billions of Chained 2009 Dollars from the BEA's NIPA accounts. Investment is calculated as gross private fixed investment plus consumption expenditures on durable goods (since durable goods behave more similar to investment than to nondurable goods consumption). Durable consumption and investment data are also expressed in billions of Chained 2009 Dollars and reported in the BEA's NIPA accounts.

These national account series (GDP, consumption and investment) are then expressed in per capita terms after dividing them by the civilian non-institutionalized population aged 16 and over from the U.S. Bureau of Labor Statistics (BLS). This population series is simply a quarterly average of monthly data. The three of them are already reported in real terms, but we also index them to $2009=100$ and express them in logs times 400. With these transformations we help express their cylical component in percentages at an annualized rate after filtering each of them (consistently with what we do to the corresponding endogenous variables in the simulated model).

Total hours worked refers to the index series $(2009=100)$ on hours of all persons in the non-farm business sector reported by the BLS. This series is expressed in per capita terms divided by the civilian non-institutionalized population aged 16 and over from the BLS and re-based to $2009=100$. Then, the series is expressed in logs times 400 prior to filtering to maintain consistency with its simulated counterpart. 
Real wages are measured as real compensation per hour in the non-farm business sector, with the index series $(2009=100)$ obtained from the BLS. This wage series is already reported in real terms, but we also express it in logs times 400 to make it consistent with the corresponding definition in the simulated model.

The price level is measured as the implicit price deflator for GDP $(2009=100)$, obtained from the BEA. The implicit GDP deflator is expressed in logs times 400 to keep it consistent with the corresponding model definition. We then calculate the inflation rate by simply computing the first differences of the series. The nominal short term interest rate corresponds to the three month Treasury Bill (secondary market) rate (henceforth, T-bill rate), obtained from the Board of Governors of the Federal Reserve System (H.15 Selected Interest Rates). The three-month T-bill rate is a quarterly average of daily data calculated on a discount basis, and reported annualized and in percentage terms. Therefore, the three-month T-bill rate does not have to be logged times 400 to keep it consistent with its counterpart in the model.

The interest rate spread is measured with the seasoned Baa corporate bond yield from Moody's relative to the yield on the 10-Year Treasury constant maturity, obtained from the Federal Reserve Bank of St. Louis. This spread is calculated as a quarterly average from monthly data, but does not have to be transformed in logs and multiplied by 400 to make it comparable with its endogenous counterpart from the model. The nonfinancial corporate leverage is computed as the ratio of the net worth (level) of the nonfinancial corporate business sector over its total assets (level) times 100, using data from the Board of Governors of the Federal Reserve System (Z.1 Financial Accounts of the U.S.). This data is reported as end-of-period, at quarterly frequency. No further adjustment of the leverage ratio series is needed.

The Business Cycle Component and its Features. We extract the cyclical component of all these series by HP-filtering them with a one-sided filter using a lambda of 1600 and a power of 2, except for the leverage ratio that is demeaned instead. We apply the same filtering to the corresponding endogenous data simulated by the model to ensure the comparability between simulated and empirical moments. Some of the selected business cycle moments reported below are used for estimation with our simulated method of moment (SMM) approach and, more generally, they provide us with an empirical point of reference for the parameterization of the model.

Main files to replicate the data and examine the business cycle moments used to parameterize the model:

1. BusinessCyclesStylizedFacts.xlsx. This excel file contains links to the data downloaded from the Federal Reserve Bank of St. Louis' FRED database as well as the necessary calculations to compute the business cycle moments reported in the paper.

2. one_sided_hp_filter_serial.m. This matlab program extracts the cyclical component from a time series using the one-sided HP-filter based on spare matrices and exploiting their pattern. This code follows the approach described in Mehra (2004). The code was written by Alexander Meyer-Gohde.

3. one_sided_hp_filter_kalman.m. This matlab program extracts the cyclical component from a time series using the one-sided HP-filter based on implementing the Kalman filter. This code follows the approach described in page 301 of Stock and Watson (1999), as written by Alexander Meyer-Gohde. 


\section{Codes}

Driver files for simulating, estimating, and examining the benchmark model and the alternative model parameterizations considered in the paper.

1. BMZ_credit_moments_cases.m. This programs simulates the pruned $3^{\text {rd }}$ order approximation in order to generate stationary distribution and the model moment statistics. There are lines in the code that allows one to change specific parameter values. The results are saved to an external file (this is actually commented out in copy of the program so that existing results are not inadvertently overwritten).

2. BMZ_credit_conditional_GIRF_positive.m. This program generates conditional GIRF. To run this program one must first have generated the stationary distribution for the relevant model (see BMZ_credit_moments_cases.m). The default take the conditional at the approximately the 5th and 95th percentiles

3. BMZ_credit_unconditional_GIRF_positive.m. This program generates unconditional GIRF. To run this program one must first have generated the stationary distribution for the relevant model (see BMZ_credit_moments_cases.m). Here 500 starting values, randomly drawn from the unconditional distribution, are fed is an initial conditions for the IRF calculations.

4. BMZ_credit_esitmate.m. This program that estimates a select number of model parameters by SMM. The current version of the program calls initial parameter values from a file (the final estimated values). If you want to start from scratch, this would need to be commented out.

Files used for printing out and plotting results:

1. BMZ_credit_conditional_GIRF_plot.m. This graphs out various impulse response experiments. The program calls external files that contain the results of previously generated IRF experiments (both unconditional and conditional).

2. BMZ_credit_conditional_dist.m. This file graphs out various scatterplots and conditional density functions based on the unconditional distribution for a specific model. Draws from the unconditional distribution are read in from an external file.

3. BMZ_credit_moments_load_print.m. This file prints out a table with various moment statistics for the alternative models. The statistics are read in from various external data files.

To run these programs the "current folder" in MATLAB must be the same folder that bgg_with_SV.mod is in.

Supplementary files that are called by the driver programs and other subroutines. These files are specific for the project in this paper:

1. bgg_with_SV.mod. This is the main Dynare file for the code. This initializes Dynare and creates the global variables that will be used later to solve for the pruned $3^{\text {rd }}$ order approximation.

2. bgg_with_SV_SS.m. This matlab file is auxiliary external code needed to calculate the steady state of the model for Dynare. This program finds initial estimate of the steady state. 
3. bgg_with_SV_Rev.m. This file allows the user to modify parameter values and to call modified Dynare subroutines that are then used to solve for $2^{\text {nd }}$ and $3^{\text {rd }}$ order approximations. These will be used to construct the pruned $3^{\text {rd }}$ order approximation.

4. bgg_with_SV_SS_Rev.m. This code solves for steady state (called from the bgg_with_SS_Rev.m subroutine).

The file bgg_with_SV.mod contains the compact set of equations that we use to simulate the model in our Dynare code (written for Dynare version 4.3.2 and Matlab R2012a (7.14.0.739)). Some preliminary considerations about the model and the Dynare code:

- Special cases of the model without frictions: The frictionless version of the model can be described with the same set of equilibrium conditions imposing certain restrictions on the parameterization of the code: $\varphi_{p}=0$ (no price adjustment costs), $\mu=0$ (no monitoring costs) and a policy rule that targets $\bar{\Pi}_{t}^{*}=1$ (setting the net inflation target to zero and the gross to one). This alone suffices to characterize the dynamics of the model without nominal rigidities and without the financial friction.

- Pre-determined variables and state variables in the model: There are two endogenous state variables in the model: capital, $K_{t}$, and real net worth, $N_{t}^{r} \equiv \frac{N_{t}}{P_{t}}$. We use the predetermined_variables option in Dynare to change the default convention which is that the timing of a variable reflects when this variable is actually decided. Hence, the endogenous variables declared as predetermined are decided one period ahead of all other endogenous variables. The convention is that $K_{t}$ is actually decided prior to $t$ with the investment decisions made at time $t-1$ and in previous periods, so it is declared as predetermined. In turn, the real net worth of entrepreneurs $N_{t}^{r}$ used to fund capital purchases at time $t$ depends on past decisions but also depends on the entrepreneurial income earned at time $t$. Given this timing, real net worth is not treated as a predetermined variable.

The following subroutines are from the "sim_folder." These subroutines are called with simulating the pruned 3rd order approximation or when generating impulse responses from the $3^{\text {rd }}$ order approximation.

1. postGIRFsim_3rd_aux.m. This subroutine generates pruned $3^{\text {rd }}$ order approximation and then calculates IRFs (given an initial condition).

2. postmoment_3rd_aux.m. This subroutine generates pruned $3^{\text {rd }}$ order approximation and the simulates and does moment calculations for the pruned $3^{\text {rd }}$ order approximation.

3. particle3rd_setup_aux.m. This subroutine constructs the components of the pruned $3^{\text {rd }}$ order approximation.

4. SIM3rd.m. Simulates the pruned $3^{\text {rd }}$ order approximation.

5. IRFSIM3rd.m. Calculates simulated estimates of the impulse responses (not currently used).

6. GIRF3rd.m. This subroutine calculates the analytical impulse responses (given an initial conditional).

7. Statemoments3rdvar.m. Calculates the unconditional mean of the model variables for pruned $3^{\text {rd }}$ order approximation. 
8. stoch_simul2.m. Custom modified Dynare subroutine.

9. dynare_solve2.m. Custom modified Dynare subroutine.

10. resol2.m. Custom modified Dynare subroutine.

11. evaluate_steady_state2.m. Custom modified Dynare subroutine.

12. k_order_pert2.m. Custom modified Dynare subroutine.

13. stochastic_solvers2.m. Custom modified Dynare subroutine.

14. mykron2.m. Custom kronecker product subroutine.

15. mykron.m. Custom kronecker product subroutine.

16. hpfilter.m. Regular 2-sided hp filter.

17. one_sided_hp_filter_kalman.m. One sided HP filter.

18. one_sided_hp_filter_serial.m. One sided HP filter.

The following files are from the GMM_folder. They are called when estimating the model by GMM/SMM.

1. SIM3rdSMM.m. This program simulates pruned $3^{\text {rd }}$ order approximation when doing SMM.

2. Estmoment.m. This subroutine calculates the empirical moment conditions used in estimation.

3. Estmoment_Wout.m. This subroutine calculates the empirical moment conditions as well as outputs the weighting matrix $W=S$.

4. GMMest_Moment_3rd.m. This subroutine generates pruned $3^{\text {rd }}$ order approximation and then calculates various moment conditions implied by the model.

5. GMMest_loss_3rd.m. Generates the empirical loss function for the GMM/SMM when using a pruned $3^{\text {rd }}$ order approximation.

6. GMMest_loss_3rd_exact.m. Generates the estimated moment conditions when using a pruned $3^{\text {rd }}$ order approximation.

7. GMMloss.m. Calculates the loss function for the moment conditions for a specific weighting matrix.

8. GMMest_Mout_3rd.m. Generates the estimated moment conditions when using a pruned $3^{\text {rd }}$ order approximation (outputs a "flag" that indicates that the model is valid).

9. GMMest_Wout_3rd.m. Generates the estimated moment conditions and outputs the weighting matrix $W=S$ for pruned $3^{r d}$ order approximation.

10. numgrad2mod.m. Slightly modified subroutine to calculated numerical $2^{\text {nd }}$ derivatives (originally based on a program written by Chris Sims).

\section{Model Variables and Parameters}




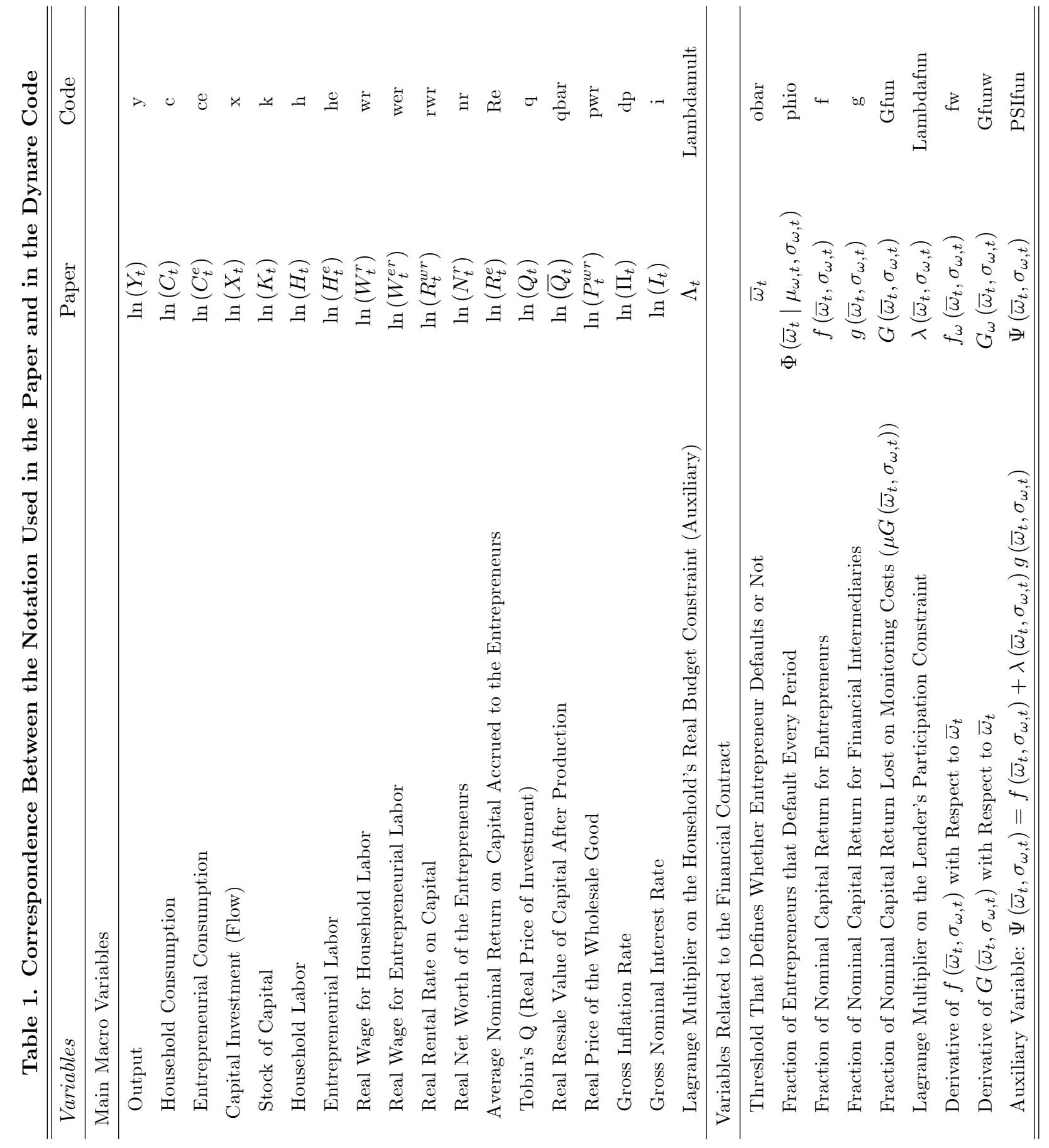




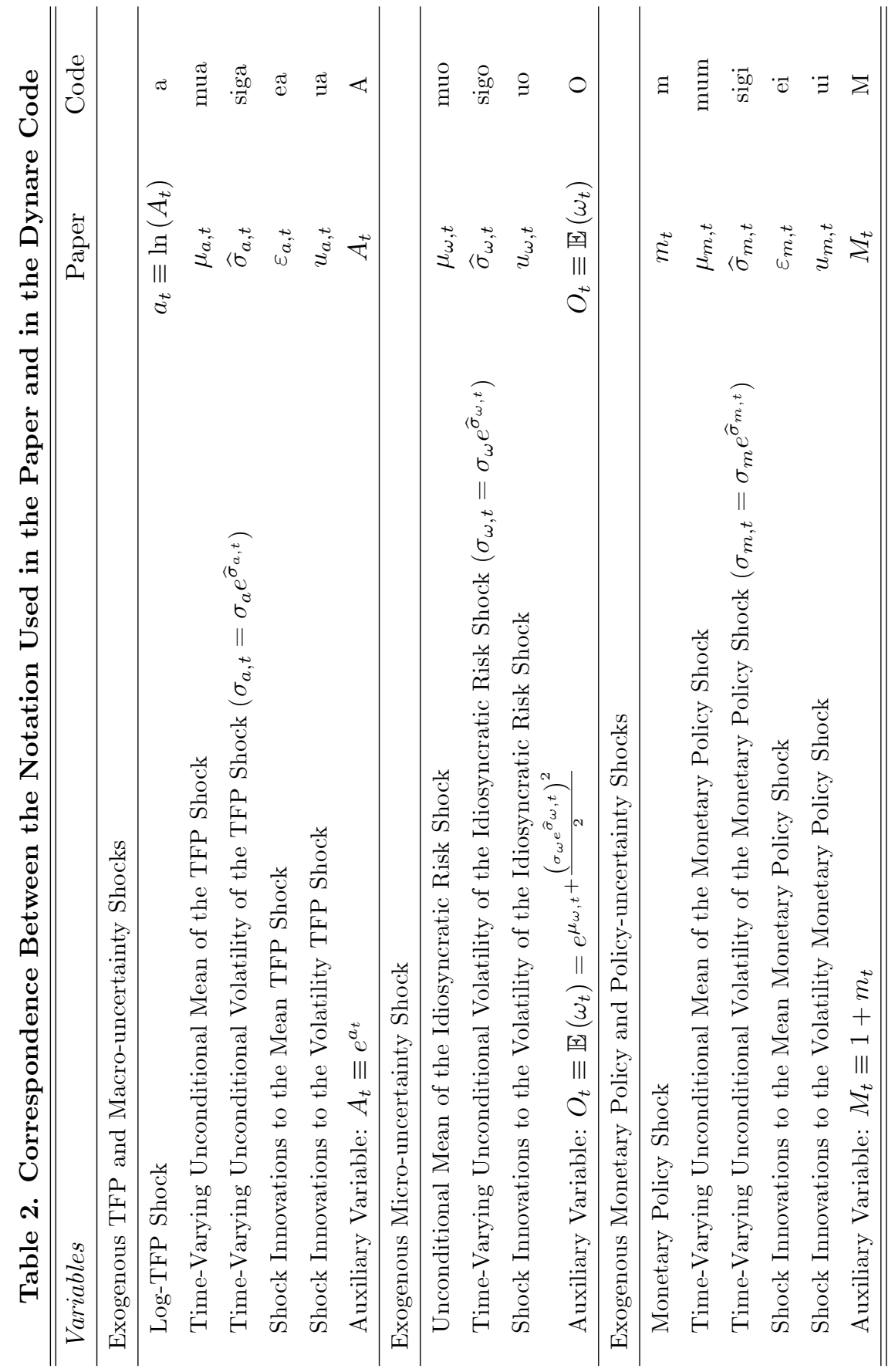




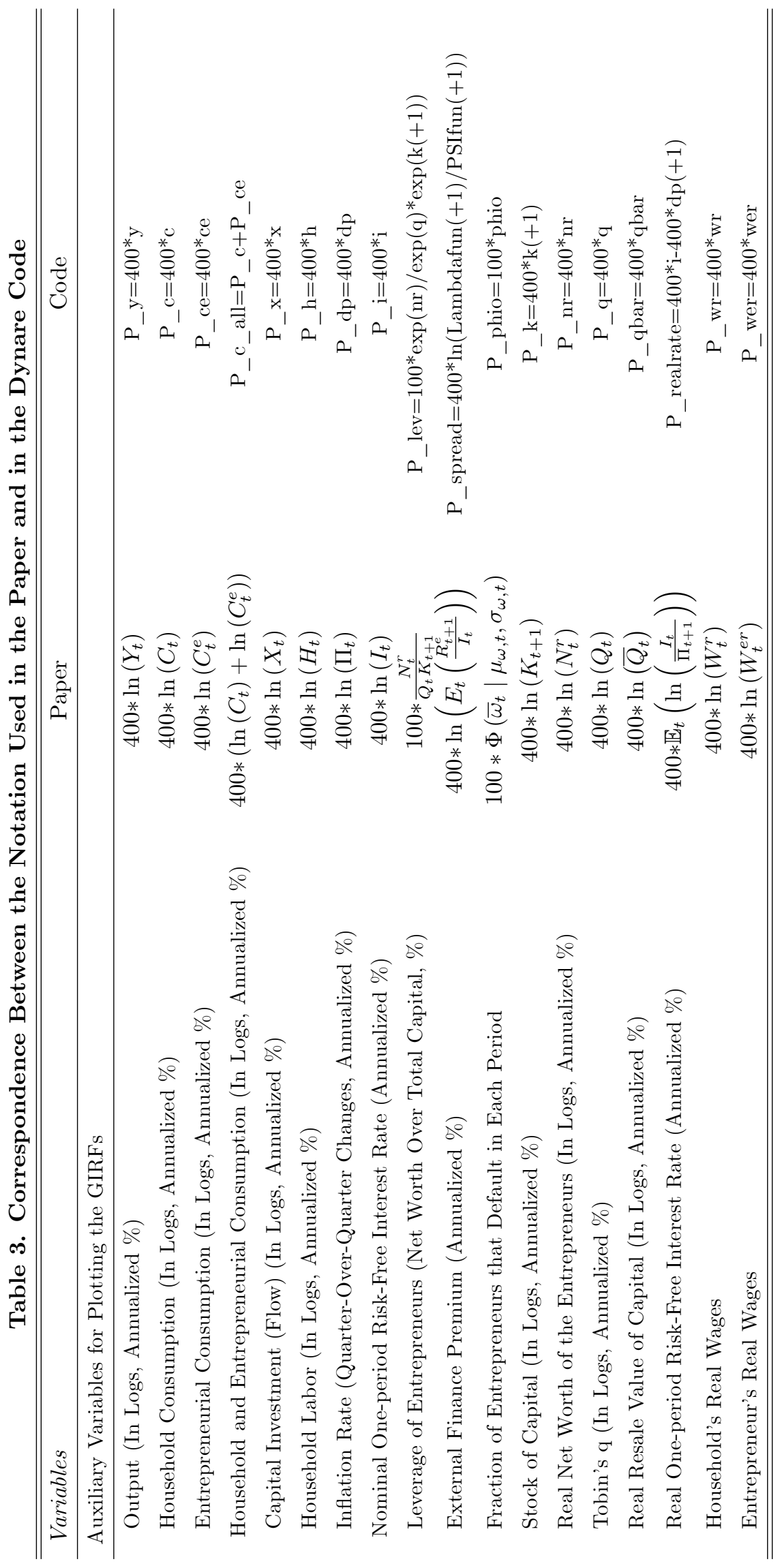




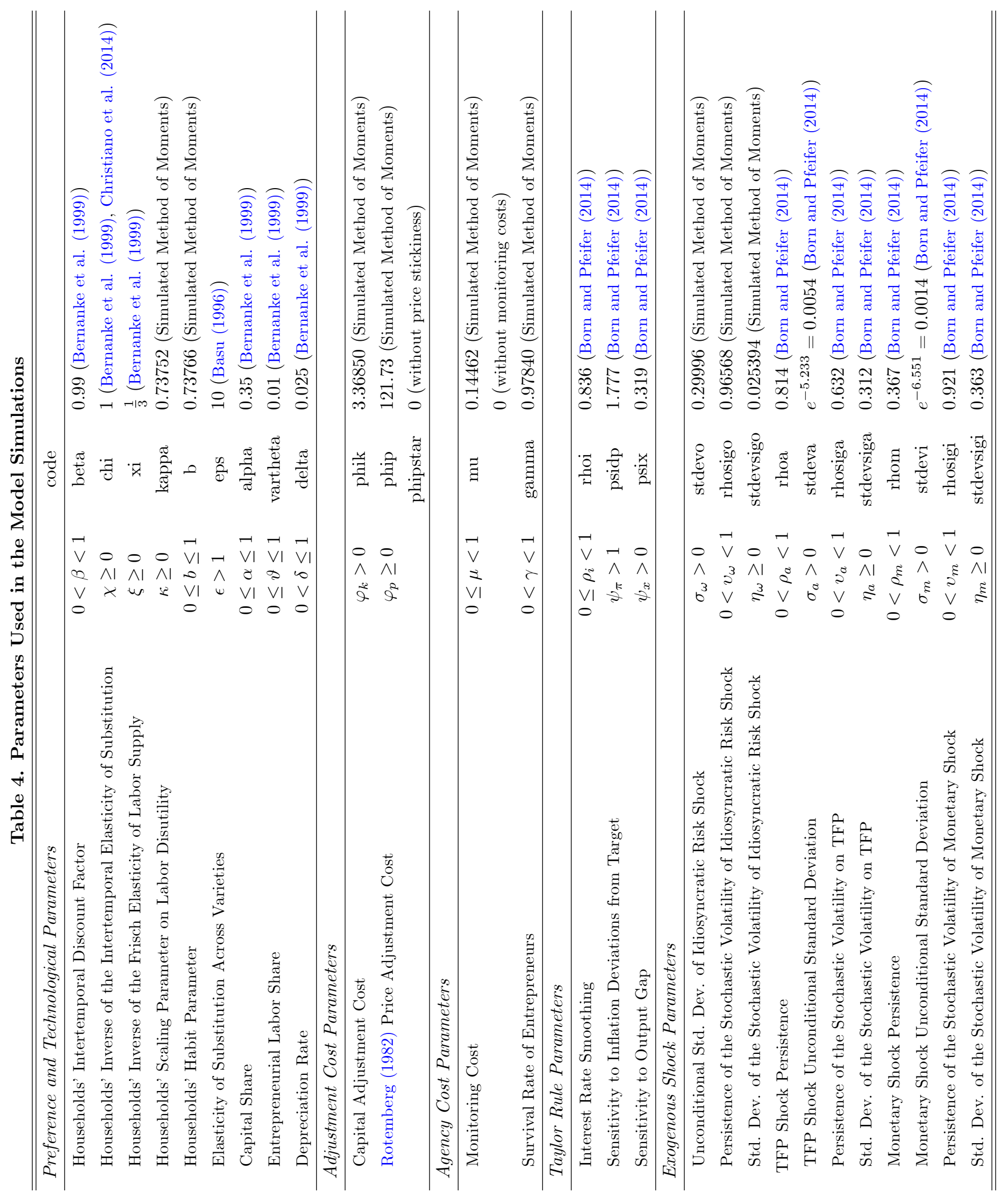




\section{$7 \quad$ Quantitative Findings}

1. Figure 1. We explore the responses of output, the spread and the nominal interest rate to a +1 shock, conditional on high uncertainty (micro-uncertainty, TFP uncertainty and monetary policy uncertainty, individually), Figure 1A captures the non-normalized responses while Figure 1B describes the response normalized by the size of each shock innovation. We show here that TFP uncertainty and monetary policy uncertainty effects are largely driven by the size of their volatility. Finally, Figure 1C shows the normalized responses for the case where we eliminate the monitoring costs $(\mu=0)$ which shuts down the financial accelerator channel. This shows that the propagation of shocks through the spread is no longer present-only micro-uncertainty matters to explain movements of the spread.

2. Figure 2. We explore the responses of output, the spread, the nominal interest rate and other key macro-finance variables (inflation, Tobin's Q, investment, the default probability, aggregate leverage, and real net worth) to a $+1 /-1$ standard deviation shock conditional on high, mid, and low values of the endogenous spread. Figure 2A plots those responses to a TFP shock, Figure 2B to a TFP stochastic volatility shock, Figure 2C to a micro-uncertainty shock, Figure 2D to an interest rate rule shock, and Figure $2 \mathrm{E}$ to an interest rate rule stochastic volatility shock. The evidence shows that the propagation of one standard deviation shocks is largely symmetric. We also note that micro-uncertainty shocks tend to have much larger effects than other stochastic volatility shocks (TFP and the monetary policy rule) - yet monetary policy uncertainty can have stronger real effects than TFP uncertainty.

3. Figure 3. We explore the responses of output, the spread, the nominal interest rate and other key macro-finance variables (inflation, Tobin's Q, investment, the default probability, aggregate leverage, and real net worth) to a $+2 /-2$ standard deviation shock conditional on high, mid, and low values of the endogenous spread. Figure 3A plots those responses to a TFP shock, Figure 3B to a TFP stochastic volatility shock, Figure 3C to a micro-uncertainty shock, Figure 3D to an interest rate rule shock, and Figure 3E to an interest rate rule stochastic volatility shock. The evidence complements that of Figure 2 showing that shocks that are twice as large (which occur less frequently too) have more sizeable effects, but generally those effects are approximately twice bigger. Therefore, findings do not appear to show that larger (and less frequent) shocks will have an effect above and beyond what one would expect given the increase in the size of the shock.

4. Figure 4. We explore the responses of output, the spread, the nominal interest rate and other key macro-finance variables (inflation, Tobin's $Q$, investment, the default probability, aggregate leverage, and real net worth) to a $+1 /-1$ standard deviation shock conditional on high, mid, and low values of the micro-uncertainty shock. Figure 4A plots those responses to a TFP shock, Figure 4B to a TFP stochastic volatility shock, Figure 4C to a micro-uncertainty shock, Figure 4D to an interest rate rule

shock, and Figure 4E to an interest rate rule stochastic volatility shock. The evidence shows that the propagation of one standard deviation shocks is largely symmetric. We also note that micro-uncertainty shocks tend to have much larger effects than other stochastic volatility shocks (TFP and the monetary policy rule) - yet monetary policy uncertainty can have stronger real effects than TFP uncertainty. Finally, we find that the results conditional on the level of micro-uncertainty are very similar to those reported based on the level of the endogenous spread (Figure 2). 
5. Figure 5. We illustrate in Figure 5A the responses of output, the spread, the nominal interest rate and other key macro-finance variables (investment, Tobin's Q, inflation, consumption, hours worked, and leverage) to a $+1 /-1$ standard deviation shock (TFP shock, TFP uncertainty, micro-uncertainty, monetary policy shock, monetary policy uncertainty) whenever the initial conditions are set equal to the unconditional mean. We illustrate in Figure 5B and Figure 5C the responses of output, the spread, the nominal interest rate to a $+1 /-1$ standard deviation shock (TFP shock, TFP uncertainty, microuncertainty, monetary policy shock, monetary policy uncertainty) whenever we consider setting the initial conditions conditional on the 5th, 50th, and 95th percentiles of the endogenous spread (Figure 5B) and whenever we consider setting the initial conditions conditional on the 1st, 50th, and 99th percentiles of the endogenous spread (Figure 5C). Our results show that the initial conditions have a modest effect on the propagation of the shocks.

6. Figure 6. We show in Figure 6A the responses of output, the spread, and the nominal interest rate to a +1 standard deviation of the shocks (TFP shock, TFP uncertainty, micro-uncertainty, monetary policy shock, monetary policy uncertainty) conditional on high spreads, under alternative specifications: the baseline compared against a model with high relative risk aversion or low intertemporal elasticity of substitution $(\chi=7)$, with no monitoring costs and no financial accelerator $(\mu=0)$, and with no nominal rigidities $\left(\varphi_{p}=0\right)$. There findings indicate that the monitoring costs and the risk aversion parameter play a significant role in the propagation of shocks and their effectiveness through the financial accelerator channel (nominal rates and spreads). In turn, nominal rigidities appear particularly important for the propagation of monetary policy and even monetary policy uncertainty shocks. Figure $6 \mathrm{~B}$ conducts the same exercise plotting the responses of output, the spread, and the nominal interest rate to a +1 standard deviation of the shocks (TFP shock, TFP uncertainty, micro-uncertainty, monetary policy shock, monetary policy uncertainty) conditional on high and low spreads, under the baseline specification and an alternative specification without micro-uncertainty stochastic volatility. This exercise comes to show that the addition of micro-uncertainty shocks adds another source of fluctuations to the model, but has otherwise only modest effects on the propagation path for the rest of the shocks (except perhaps on the effects it has on the spread). We interpret this as showing that augmenting the model with micro-uncertainty does not alter all that much what we know about the propagation of other shocks. Figure 6C considers another experiment where we report the responses of output, the spread, and the nominal interest rate to a +1 standard deviation of the shocks (TFP shock, TFP uncertainty, micro-uncertainty, monetary policy shock, monetary policy uncertainty) conditional on high spreads, and high uncertainty (micro-uncertainty, TFP uncertainty, and monetary policy uncertainty) individually. Our findings most notably reveal that periods of high macro (TFP) uncertainty lead to significantly larger effects of TFP level shocks on real economic activity than in any other circumstance. Similarly, periods of high monetary policy uncertainty lead to significantly larger effects of the monetary policy shock on real economic activity (almost twice as large on impact) than in any other circumstance. Figure 6D provides another illustrative experiment where the responses of output, the spread, and the nominal interest rate to a +1 standard deviation of the shocks (TFP shock, TFP uncertainty, micro-uncertainty, monetary policy shock, monetary policy uncertainty) conditional on all three stochastic volatility shocks (micro-uncertainty, TFP, and monetary policy) being either high ( $>$ 80th percentile) or low $(<20$ th percentile) simultaneously. Our findings show that periods of 
high uncertainty across all stochastic volatility shocks and low uncertainty have only modest effects on the propagation of uncertainty shocks themselves. However, our evidence suggests that the response of real economic activity to TFP level shocks is markedly stronger in periods of high overall uncertainty and, similarly, that the response to monetary policy shocks can be more than twice as large during periods of high overall uncertainty.

7. Figure 7. We show in Figure 7 the responses of output, the spread, the nominal interest rate, and other macro-finance variables (investment, Tobin's Q, inflation, consumption, hours worked, and leverage) to a +1 standard deviation of each one of the shocks (TFP shock, TFP uncertainty, microuncertainty, monetary policy shock, monetary policy uncertainty) conditional on mid-values of the endogenous spreads, under alternative specifications: the baseline compared against a model with high relative risk aversion or low intertemporal elasticity of substitution $(\chi=7)$, with no monitoring costs and no financial accelerator $(\mu=0)$, and with no nominal rigidities $\left(\varphi_{p}=0\right)$. Our findings show that reducing nominal rigidities tends to mitigate the real effects of TFP shocks while high risk-aversion amplifies those effects for mid-values of the endogenous spread (Figure 7A), while the responses to TFP uncertainty are rather modest across model specifications (Figure 7B). We also show that micro-uncertainty and its propagation depends crucially on the presence of a financial accelerator channel (Figure 7C) - hence, the interaction between credit frictions and micro-uncertainty is a crucial aspect of our model. Not surprisingly, the propagation of monetary policy shocks depends critically on the presence of nominal rigidities in the model (Figure 7D) and similarly for the propagation of monetary policy uncertainty (Figure 7E). The propagation of monetary policy uncertainty and its real effects appear to become amplified whenever the risk aversion is high-interestingly, the monetary policy uncertainty shock appears to have some effect even when there are no nominal rigidities mainly because it still influences the endogenous spreads implied by the nominal financial contract.

8. Figure 8. We show in Figure 8 the responses of output, the spread, the nominal interest rate, and other macro-finance variables (investment, Tobin's $Q$, inflation, consumption, hours worked, and leverage) to a +1 standard deviation of each one of the shocks (TFP shock, TFP uncertainty, micro-uncertainty, monetary policy shock, monetary policy uncertainty) conditional on high-values of the endogenous spreads, under alternative specifications: the baseline compared against a model with high relative risk aversion or low intertemporal elasticity of substitution $(\chi=7)$, with no monitoring costs and no financial accelerator $(\mu=0)$, and with no nominal rigidities $\left(\varphi_{p}=0\right)$. Our findings show that reducing nominal rigidities tends to mitigate the real effects of TFP shocks while high risk-aversion amplifies those effects for mid-values of the endogenous spread (Figure 8A), while the responses to TFP uncertainty are rather modest across model specifications (Figure 8B). We also show that microuncertainty and its propagation depends crucially on the presence of a financial accelerator channel (Figure 8C)-hence, the interaction between credit frictions and micro-uncertainty is a crucial aspect of our model. Not surprisingly, the propagation of monetary policy shocks depends critically on the presence of nominal rigidities in the model (Figure 8D) and similarly for the propagation of monetary policy uncertainty (Figure 7E). Qualitatively these results are the same as those reported in Figure 7 conditional on mid-values of the endogenous spread, except that we observe high spreads tend to attenuate the effect of TFP level shocks and amplify somewhat the effect of the monetary policy shock itself. In turn, the effect of high spreads on the propagation of all forms of uncertainty is rather modest. 
9. Figure 9. We show in Figure 9 the responses of output, the spread, the nominal interest rate, and other macro-finance variables (investment, Tobin's Q, inflation, consumption, hours worked, and leverage) to a +1 standard deviation of each one of the shocks (TFP shock, TFP uncertainty, micro-uncertainty, monetary policy shock, monetary policy uncertainty) conditional on high-values of the endogenous spread, high-values of the nominal interest rate, and high-values of the leverage ratio. Figure 9A suggests that the response of the spread to TFP level shocks might be somewhat muted with high interest rates, while Figure 9B indicates that there are only small differences in the transmission of TFP uncertainty shocks. Figure $9 \mathrm{C}$ shows that the propagation of micro-uncertainty shocks is somewhat more muted with high leverage and particularly with high interest rates. Figure 9D suggests that monetary policy shocks are amplified with high interest rates and dampened somewhat with high leverage, while Figure 9E indicates that the differences in the transmission of monetary policy uncertainty are rather modest.

10. Figure 10. We show in Figure 10 the responses of output, the spread, the nominal interest rate, and other macro-finance variables (investment, Tobin's Q, inflation, consumption, hours worked, and leverage) to a +1 standard deviation of each one of the shocks (TFP shock, TFP uncertainty, microuncertainty, monetary policy shock, monetary policy uncertainty) conditional on high-values of the endogenous spread, high-values of TFP uncertainty, high-values of interest rate uncertainty, and highvalues of the micro-uncertainty. Figure 10A suggests that the real effects of a TFP level shock might be amplified with high TFP uncertainty, while Figure 10B indicates that there are only small differences in the transmission of TFP uncertainty shocks. Figure 10C shows that the propagation of micro-uncertainty shocks through the endogenous spread is somewhat more muted with high interest rate uncertainty and high TFP uncertainty. Figure 10D suggests that monetary policy shocks are significantly amplified with high interest rate uncertainty, while Figure 10E indicates that the differences in the transmission of monetary policy uncertainty are rather modest with small amplification effects arising from high interest rate uncertainty.

11. Figure 11. We show in Figure 11 the probability density of the volatility of TFP, the TFP level, the micro-uncertainty volatility, the leverage ratio, the monetary policy rule volatility, and the interest rate, all of them conditional on the endogenous spread: unconditional, conditional on 5th percentile of the endogenous spread, and conditional on the 95th percentile of the spread. The density is smoothed by the Epanechnikov kernel. Our findings illustrate the differences between the different percentiles of the conditioning variable (the spread) - most notably, we show that the distribution of the microuncertainty volatility is shifted to the left and that of the nominal interest rate is shifted to the right whenever the endogenous spread is conditioned at the 5 th percentile.

12. Figure 12. We show in Figure 12 the joint distribution of the endogenous spread with a number of relevant macro-finance variables. Figure 12A illustrates the joint distribution between the endogenous spread and TFP, Figure 12B the joint distribution between the endogenous spread and the nominal interest rate, Figure 12C the joint distribution of the endogenous spread and TFP stochastic volatility (TFP uncertainty), Figure 12D the joint distribution of the endogenous spread and the interest rate stochastic volatility (monetary policy uncertainty), Figure 12E the joint distribution of the endogenous spread and micro-uncertainty, Figure $12 \mathrm{~F}$ the joint distribution of the endogenous spread and 
capital, Figure 12G the joint distribution of the endogenous spread and real net worth, and Figure $12 \mathrm{H}$ the joint distribution of the endogenous spread and output. We observe that there is a noticeable nonlinearity in the relationship between the endogenous spread and the nominal interest rate as well as in the relationship between the endogenous spread and micro-uncertainty. Moreover, we also find nonlinearities in the relationship between the endogenous spread and both capital and output.

13. Figure 13. We show in Figure 13 the joint distribution of the endogenous spread with a number of relevant macro-finance variables whenever the households' inverse of the intertemporal elasticity of substitution is increased to $\chi=7$. Figure 13A illustrates the joint distribution between the endogenous spread and TFP, Figure 13B the joint distribution between the endogenous spread and the nominal interest rate, Figure 13C the joint distribution of the endogenous spread and TFP stochastic volatility (TFP uncertainty), Figure 13D the joint distribution of the endogenous spread and the interest rate stochastic volatility (monetary policy uncertainty), Figure 13E the joint distribution of the endogenous spread and micro-uncertainty, Figure 13F the joint distribution of the endogenous spread and capital, Figure $13 \mathrm{G}$ the joint distribution of the endogenous spread and real net worth, and Figure $13 \mathrm{H}$ the joint distribution of the endogenous spread and output. We note that the increase in the parameter $\chi$ (which also determines the risk aversion) produces similar results to those reported under the baseline in Figure 12. It's worth noticing that higher values of $\chi$ lead to a higher frequency of zero-lower bound occurrences whereby the interest rate may fall near or below zero. Moreover, we also see that low interest rate episodes tend to be more strongly associated with periods of high endogenous spreads. 
Figure 1A. Generalized Impulse Response Functions: Response to a +1 Shock, Conditional on High Uncertainty, Individually (Non-Normalized).
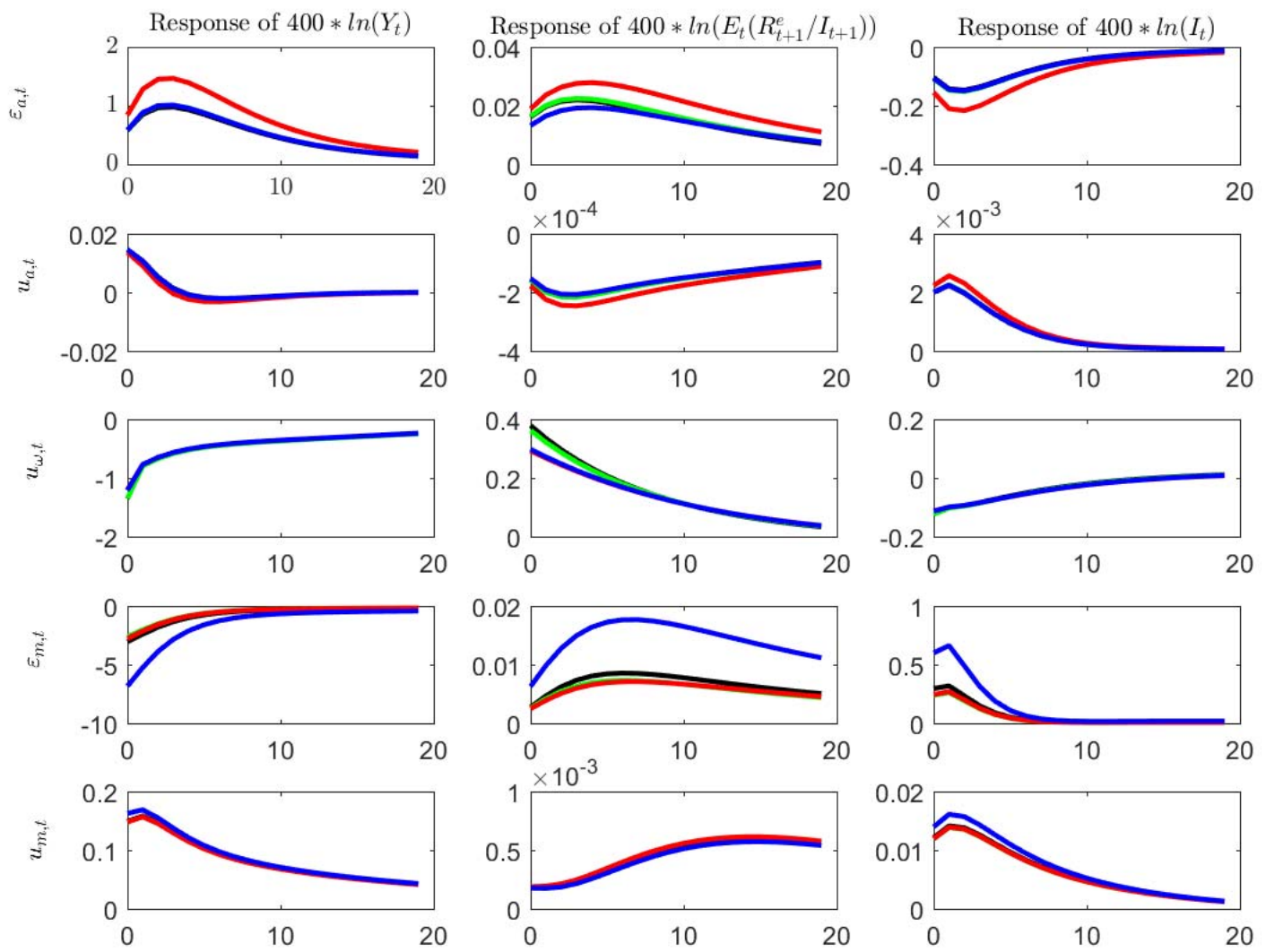

High Spread $=$ High micro uncertainty 
Figure 1B. Generalized Impulse Response Functions: Response to a +1 Shock, Conditional on High Uncertainty, Individually (Normalized by Initial Size of the Underlying Shock).
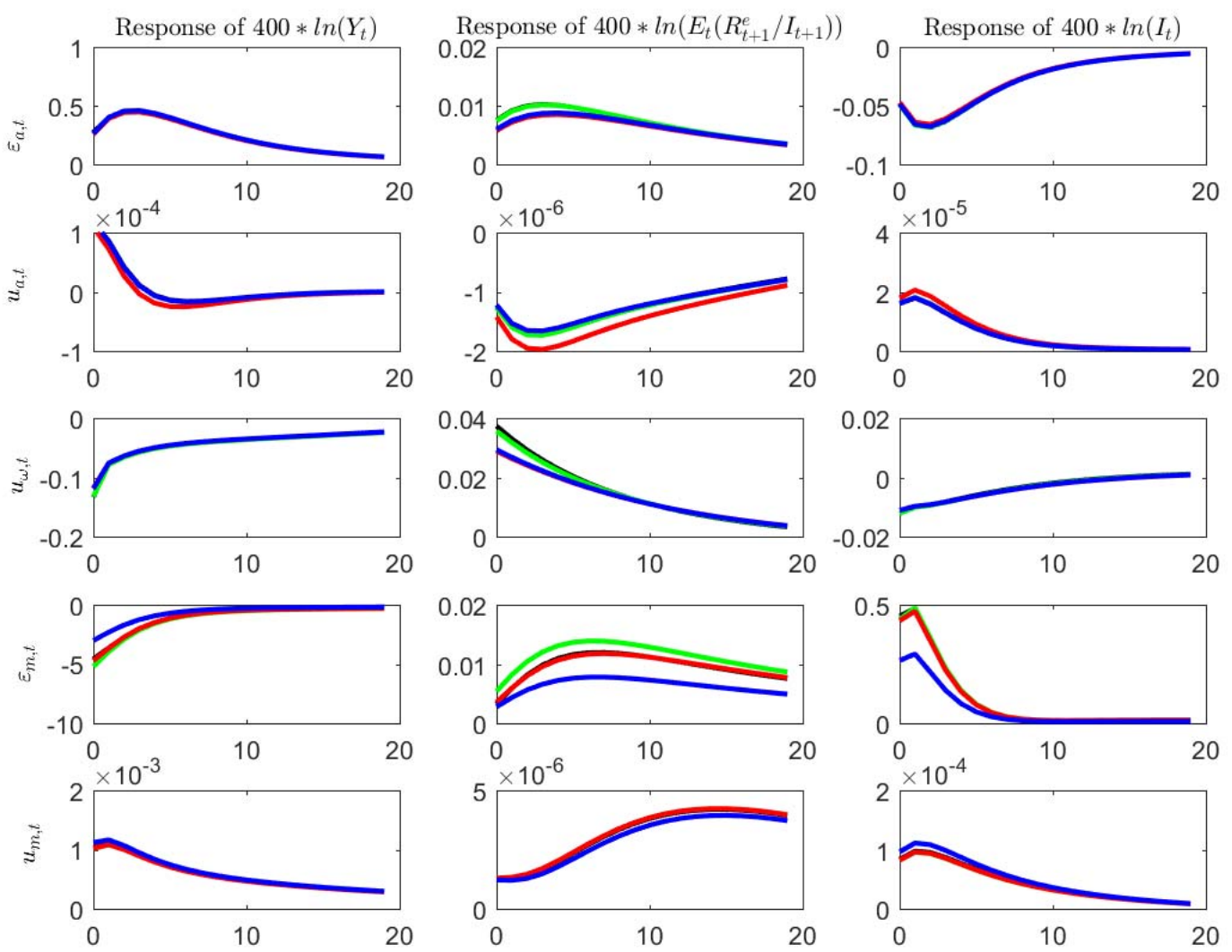

High Spread $=$ High micro uncertainty 
Figure 1C. Generalized Impulse Response Functions: Response to a +1 Shock, Conditional on High Uncertainty, Individually (Normalized by Initial Size of the Underlying Shock) whenever Default Costs Are Set to Zero $(\mu=0)$ in Order to Abstract from the Financial Accelerator Mechanism in the Model.
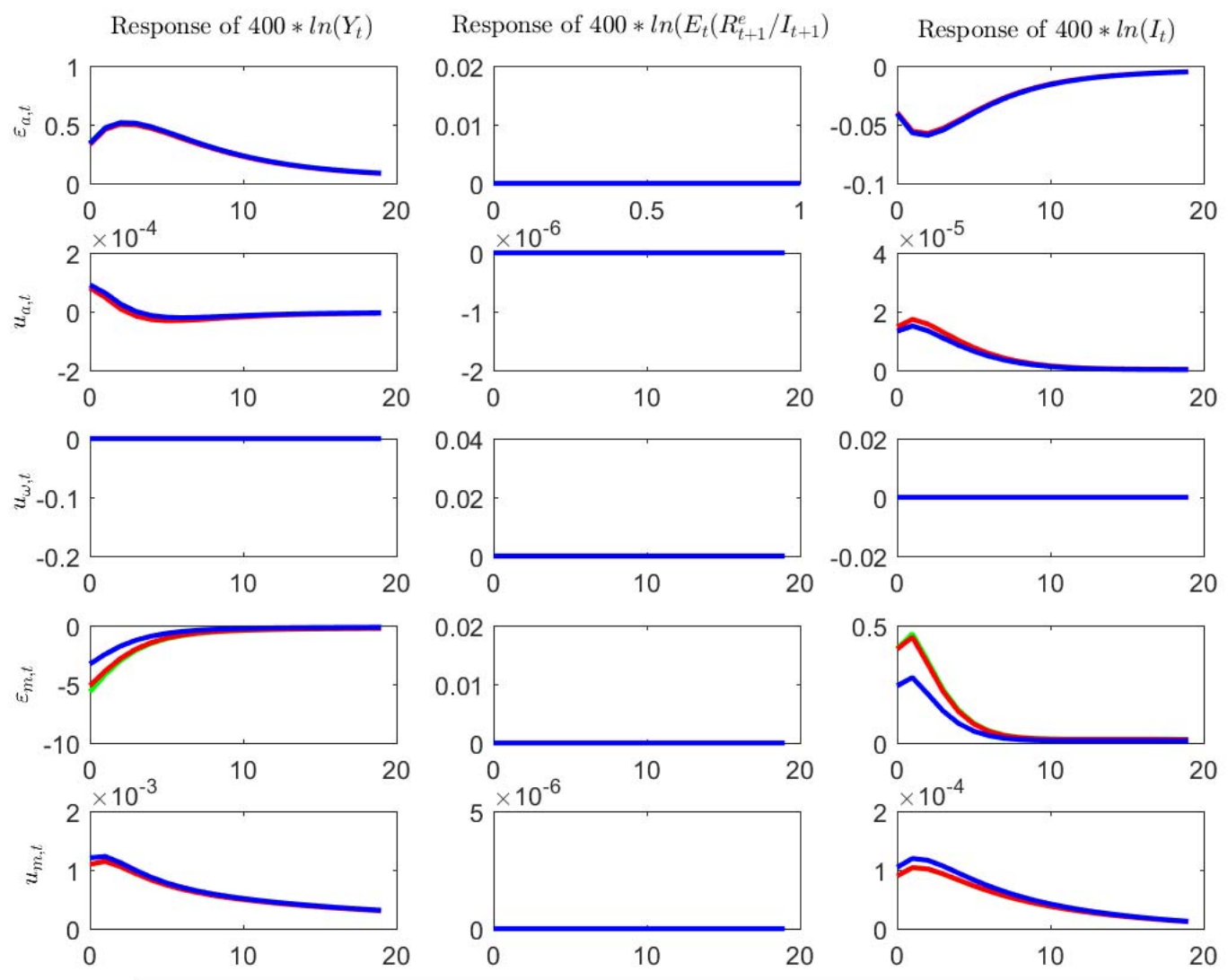

High micro uncertainty $\longrightarrow$ High TFP uncertainty — High monetary uncertainty 
Figure 2A. Generalized Impulse Response Functions: Response to a +1/-1 Standard Deviation TFP Shock, Conditional on High, Mid, Low Values of the Endogenous Spread.
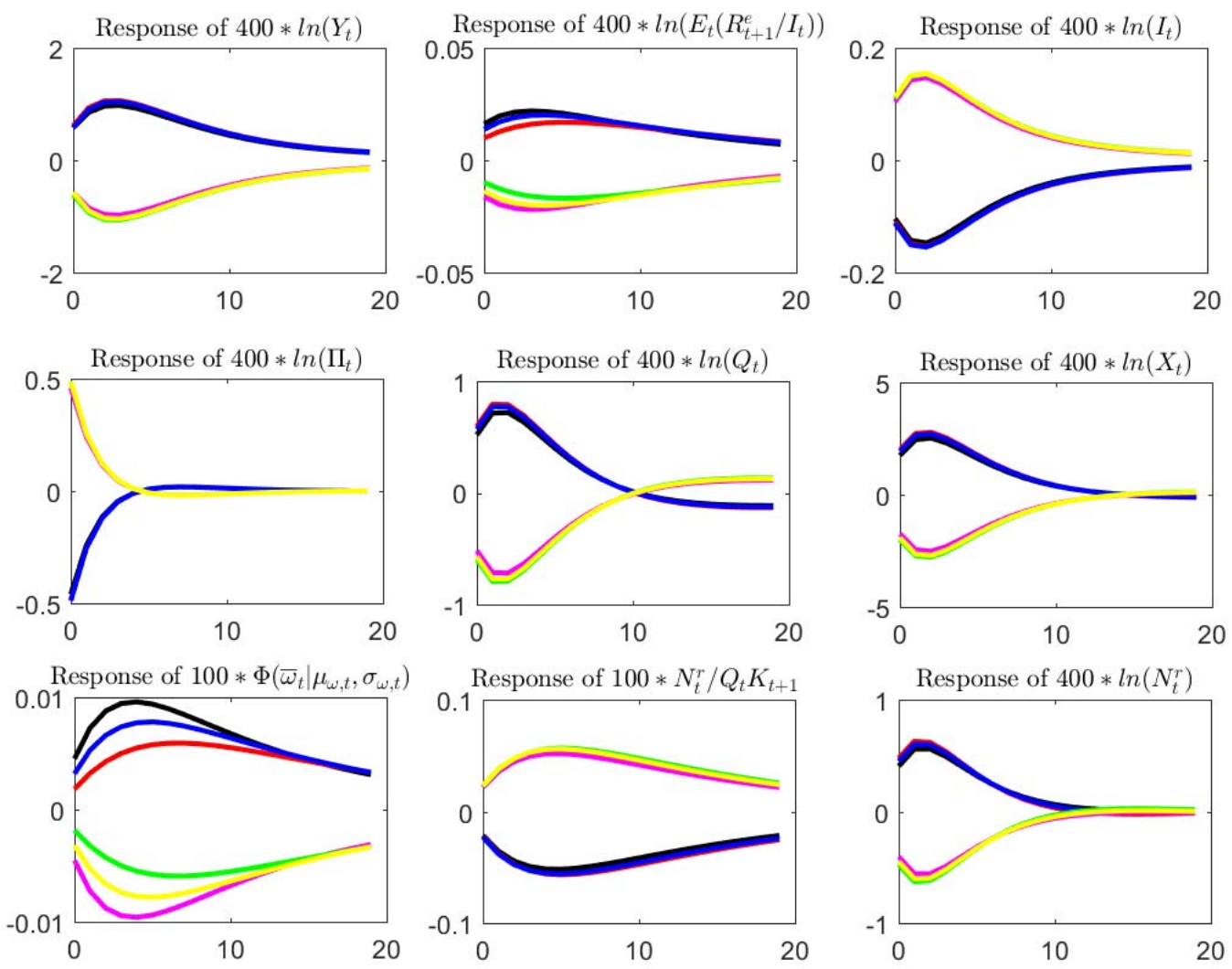
Figure 2B. Generalized Impulse Response Functions: Response to a $+1 /-1$ Standard Deviation TFP Stochastic Volatility Shock, Conditional on High, Mid, Low Values of the Endogenous Spread.
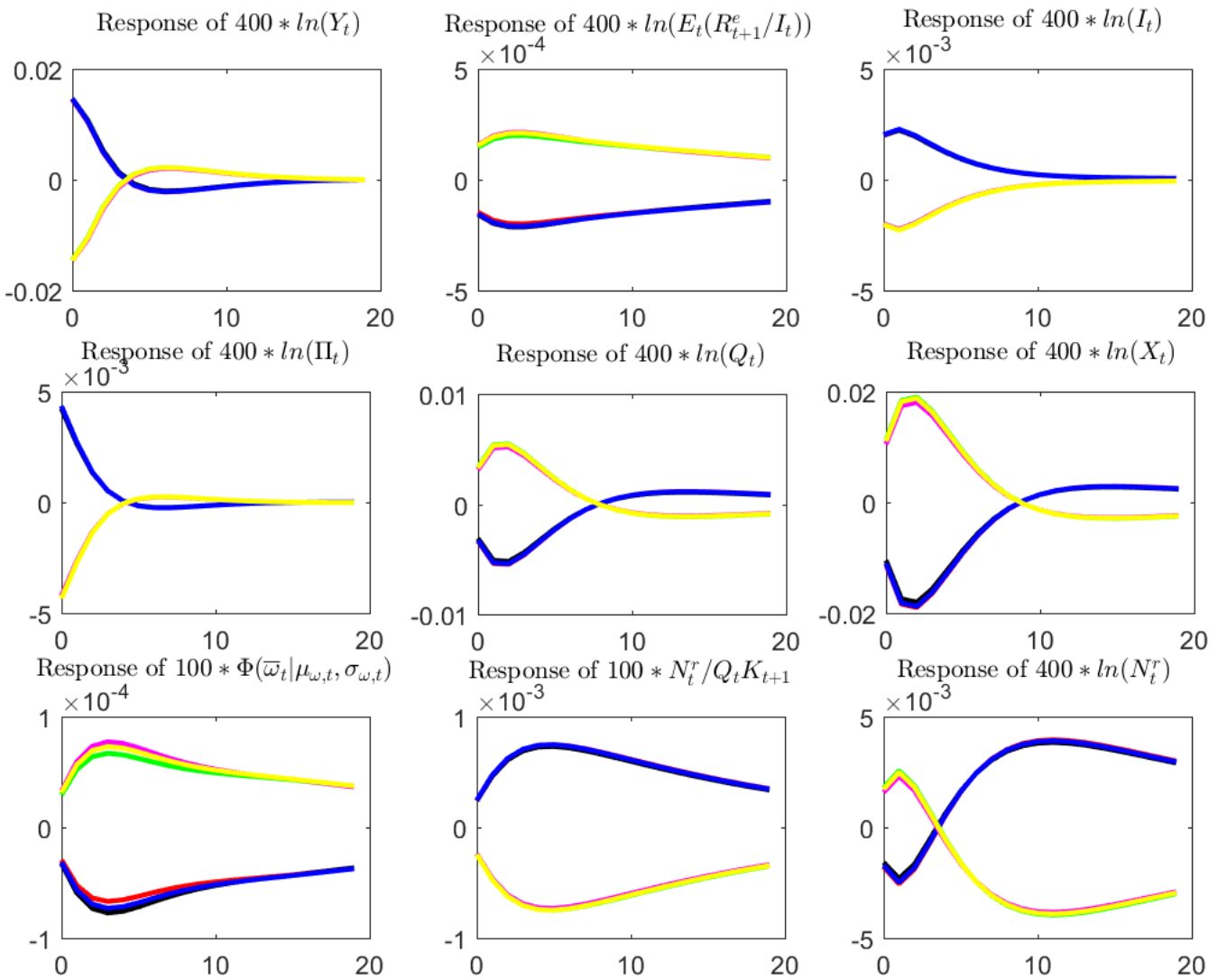
Figure 2C. Generalized Impulse Response Functions: Response to a $+1 /-1$ Standard Deviation Micro-Uncertainty Shock, Conditional on High, Mid, Low Values of the Endogenous Spread.
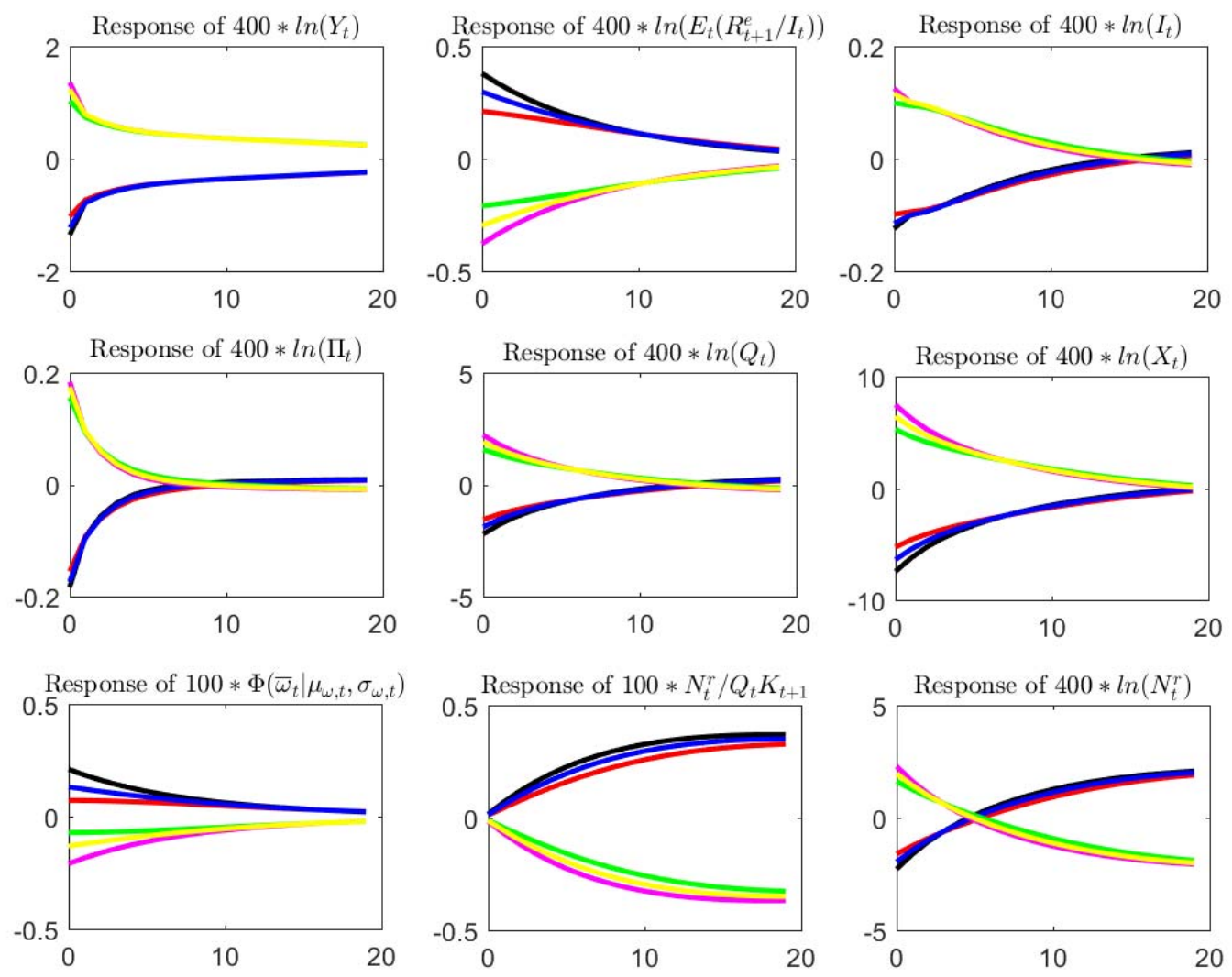
Figure 2D. Generalized Impulse Response Functions: Response to a $+1 /-1$ Standard Deviation Interest Rate Rule Shock, Conditional on High, Mid, Low Values of the Endogenous Spread.
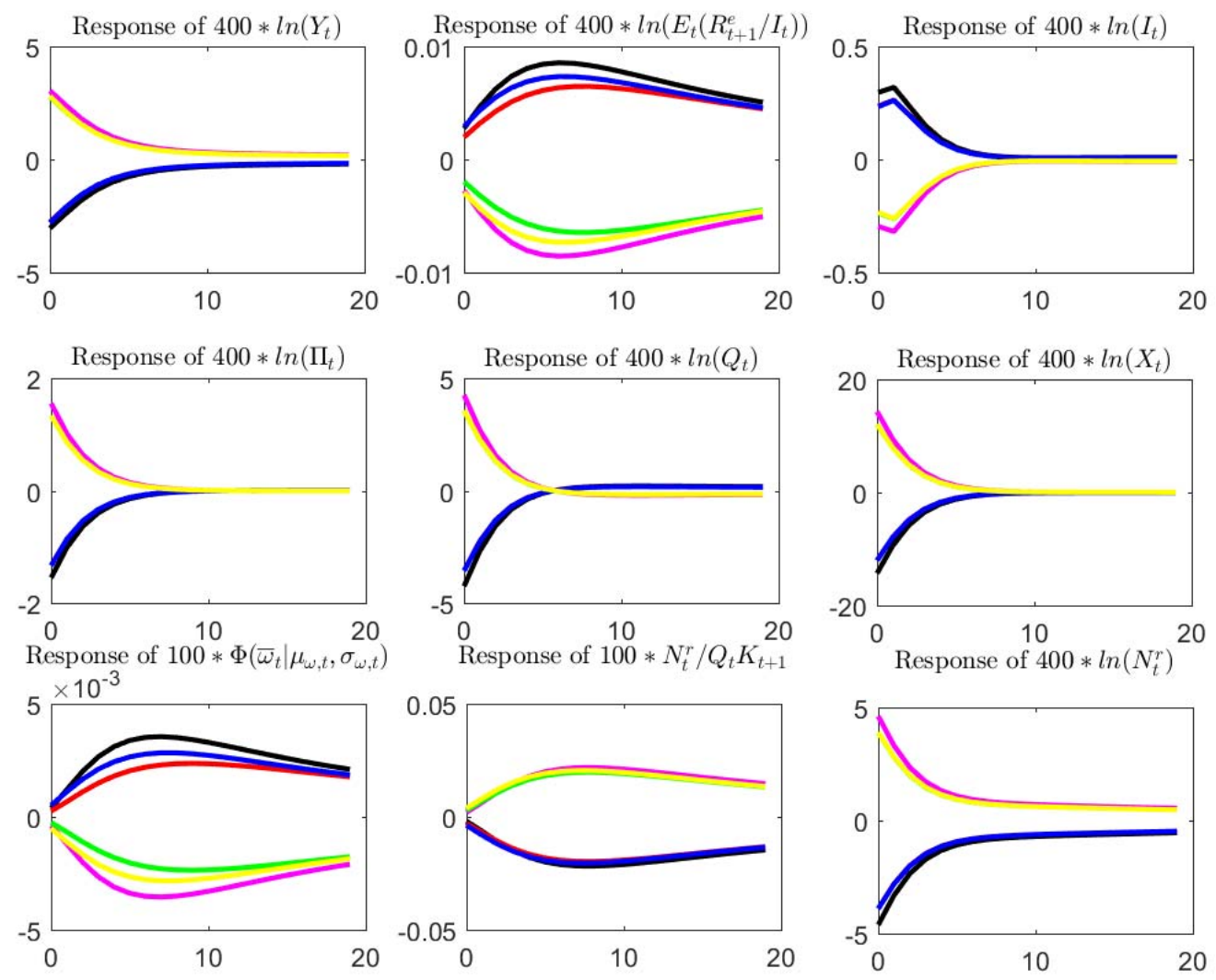

+1 sd - high $\longrightarrow+1$ sd - low

$+1 \mathrm{sd}-$ medium $--1 \mathrm{sd}$ - high

-1 sd - low

$-1 \mathrm{sd}$ - medium 
Figure 2E. Generalized Impulse Response Functions: Response to a $+1 /-1$ Standard Deviation Interest Rate Rule Stochastic Volatility Shock, Conditional on High, Mid, Low Values of the Endogenous Spread.
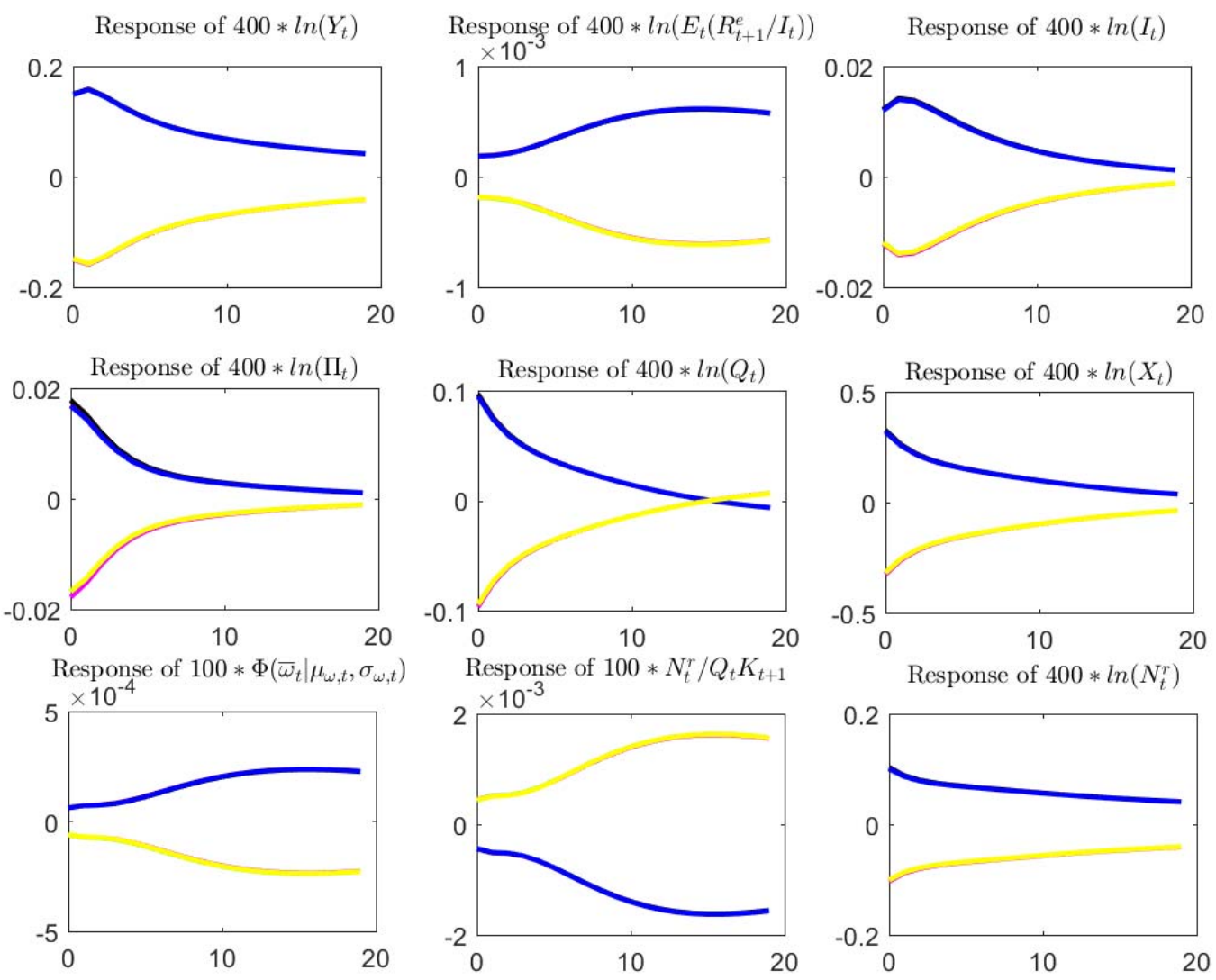

+1 sd - high -+1 sd - low

+1 sd - medium $\longrightarrow-1$ sd - high

-1 sd - low

-1 sd - medium 
Figure 3A. Generalized Impulse Response Functions: Response to a +2/-2 Standard Deviation TFP Shock, Conditional on High, Mid, Low Values of the Endogenous Spread.
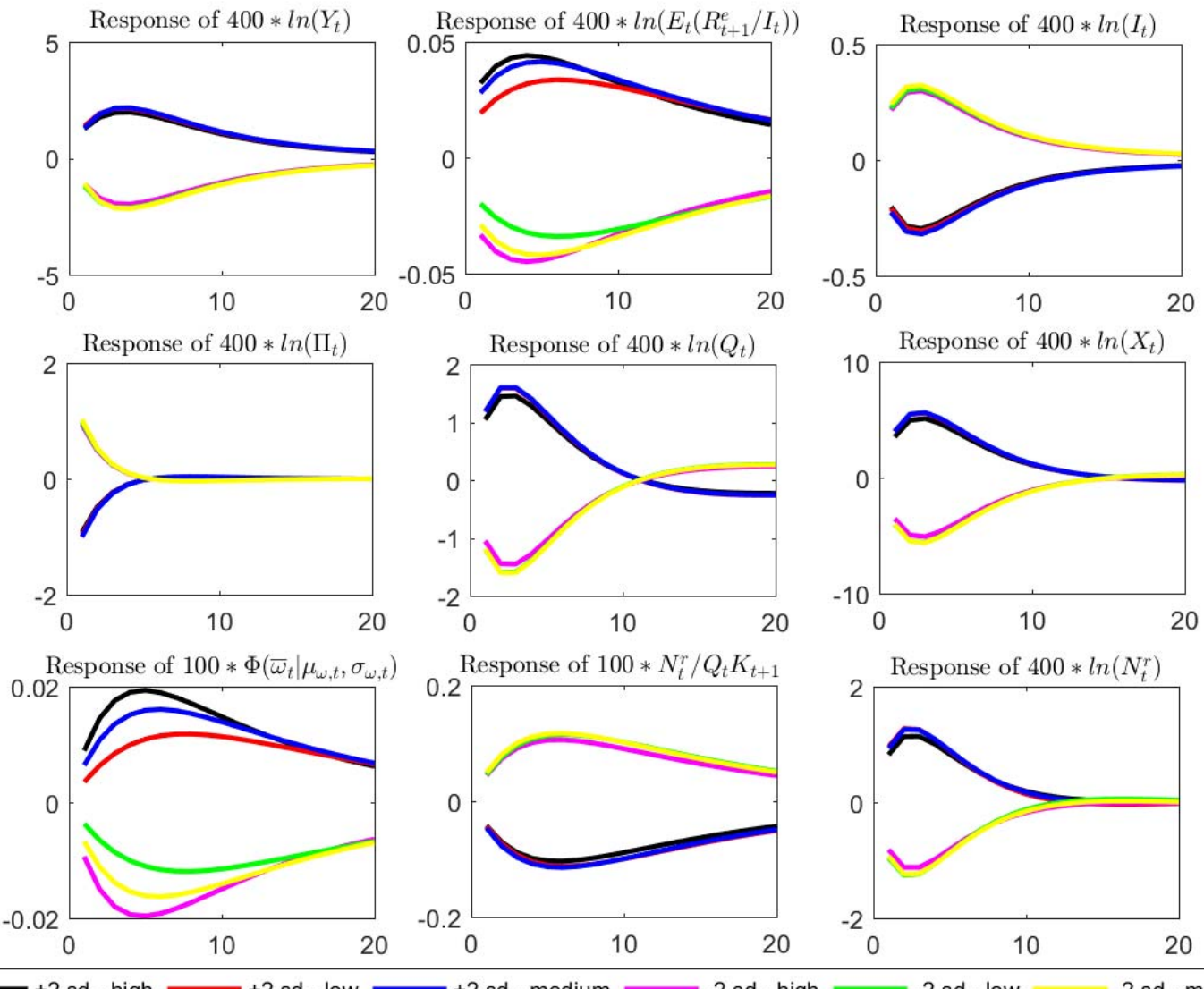

$+2 \mathrm{sd}$ - high $\longrightarrow+2 \mathrm{sd}-$ low

$+2 \mathrm{sd}-$ medium $-2 \mathrm{sd}-$ high

$-2 \mathrm{sd}-$ low $-2 \mathrm{sd}-$ medium 
Figure 3B. Generalized Impulse Response Functions: Response to a +2/-2 Standard Deviation TFP Stochastic Volatility Shock, Conditional on High, Mid, Low Values of the Endogenous Spread.
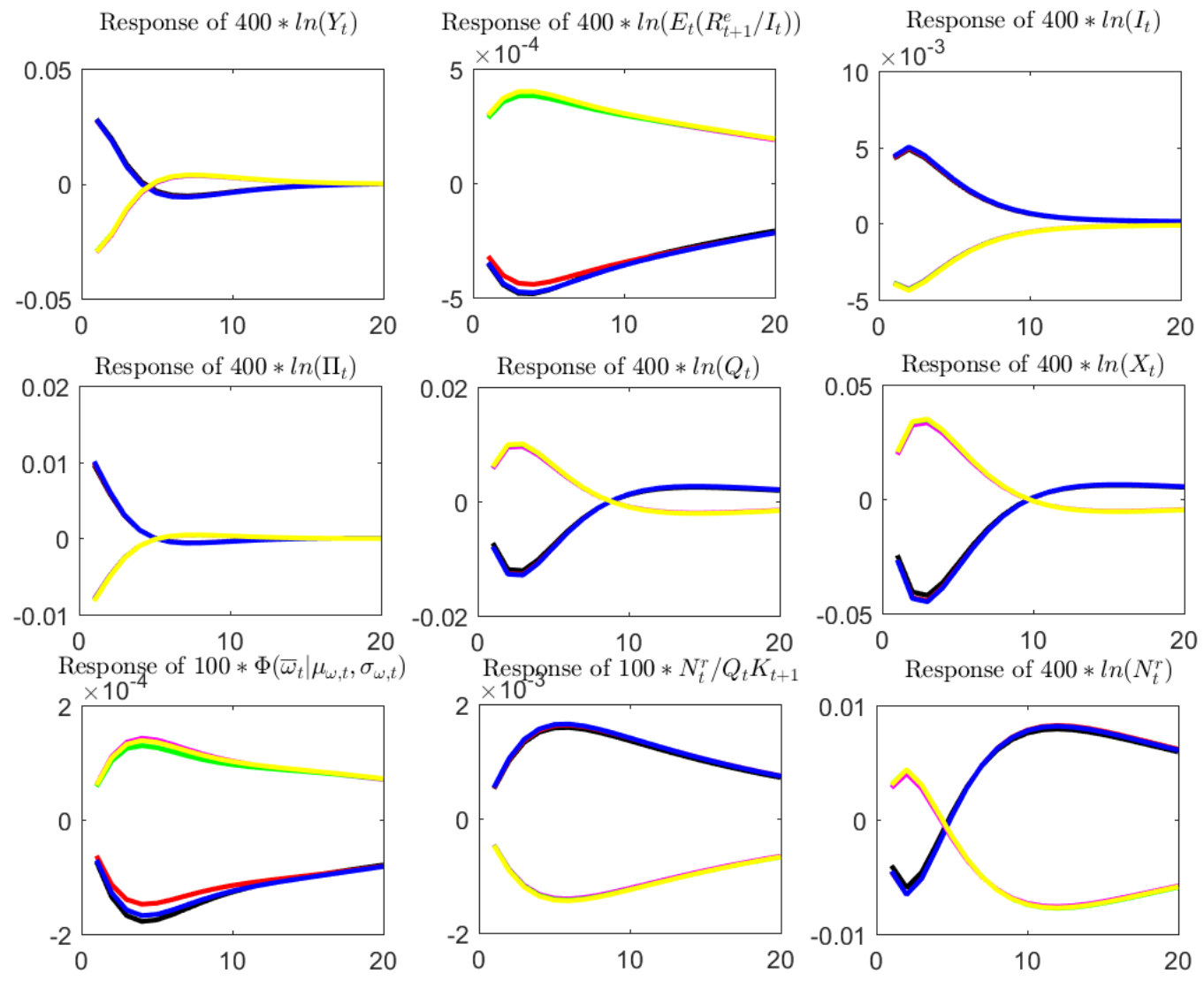

$+2 \mathrm{sd}-$ high $-+2 \mathrm{sd}-$ low

$+2 \mathrm{sd}$ - medium $--2 \mathrm{sd}$ - high

-2 sd - low

$-2 \mathrm{sd}$ - medium 
Figure 3C. Generalized Impulse Response Functions: Response to a +2/-2 Standard Deviation Micro-Uncertainty Shock, Conditional on High, Mid, Low Values of the Endogenous Spread.
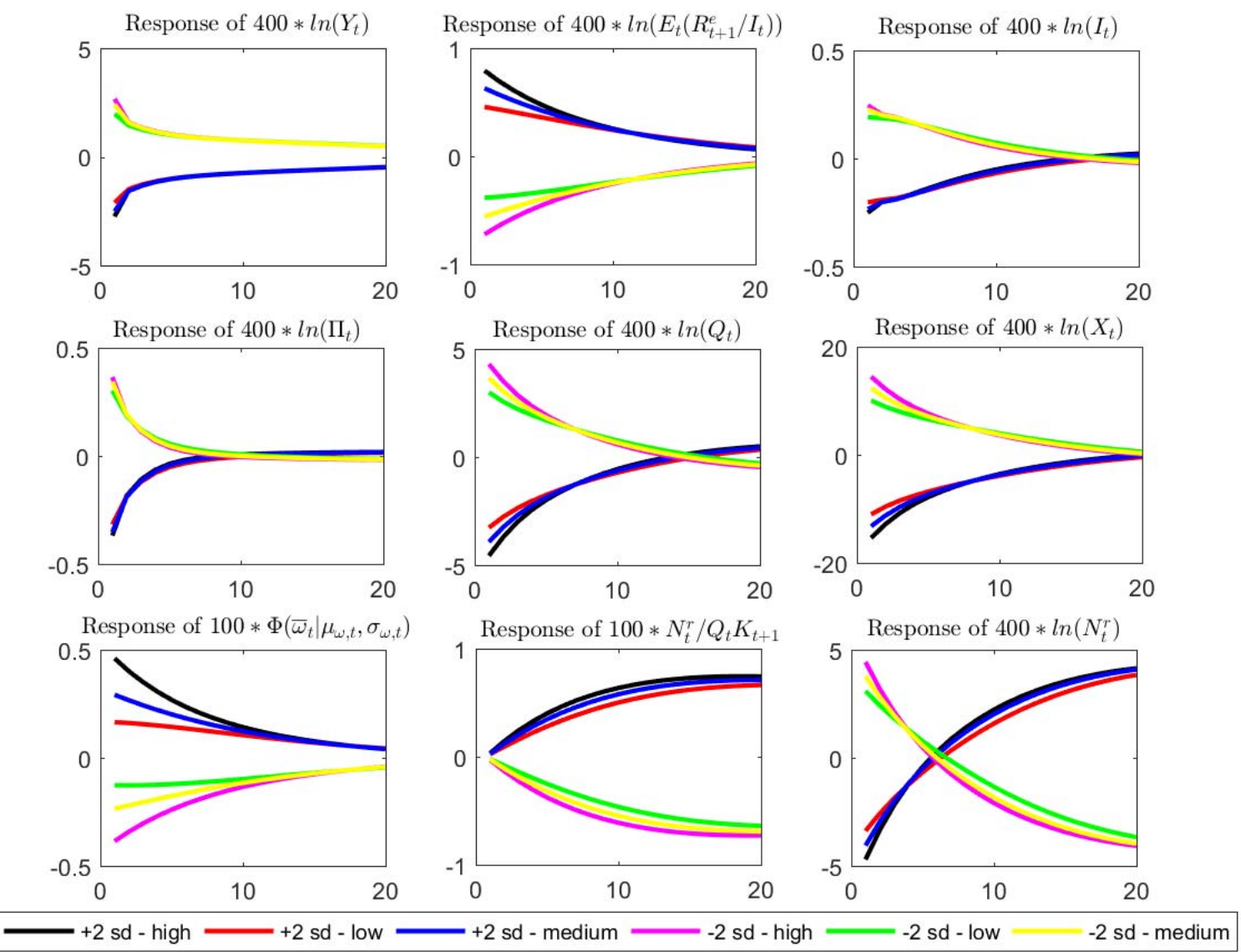
Figure 3D. Generalized Impulse Response Functions: Response to a +2/-2 Standard Deviation Interest Rate Rule Shock, Conditional on High, Mid, Low Values of the Endogenous Spread.
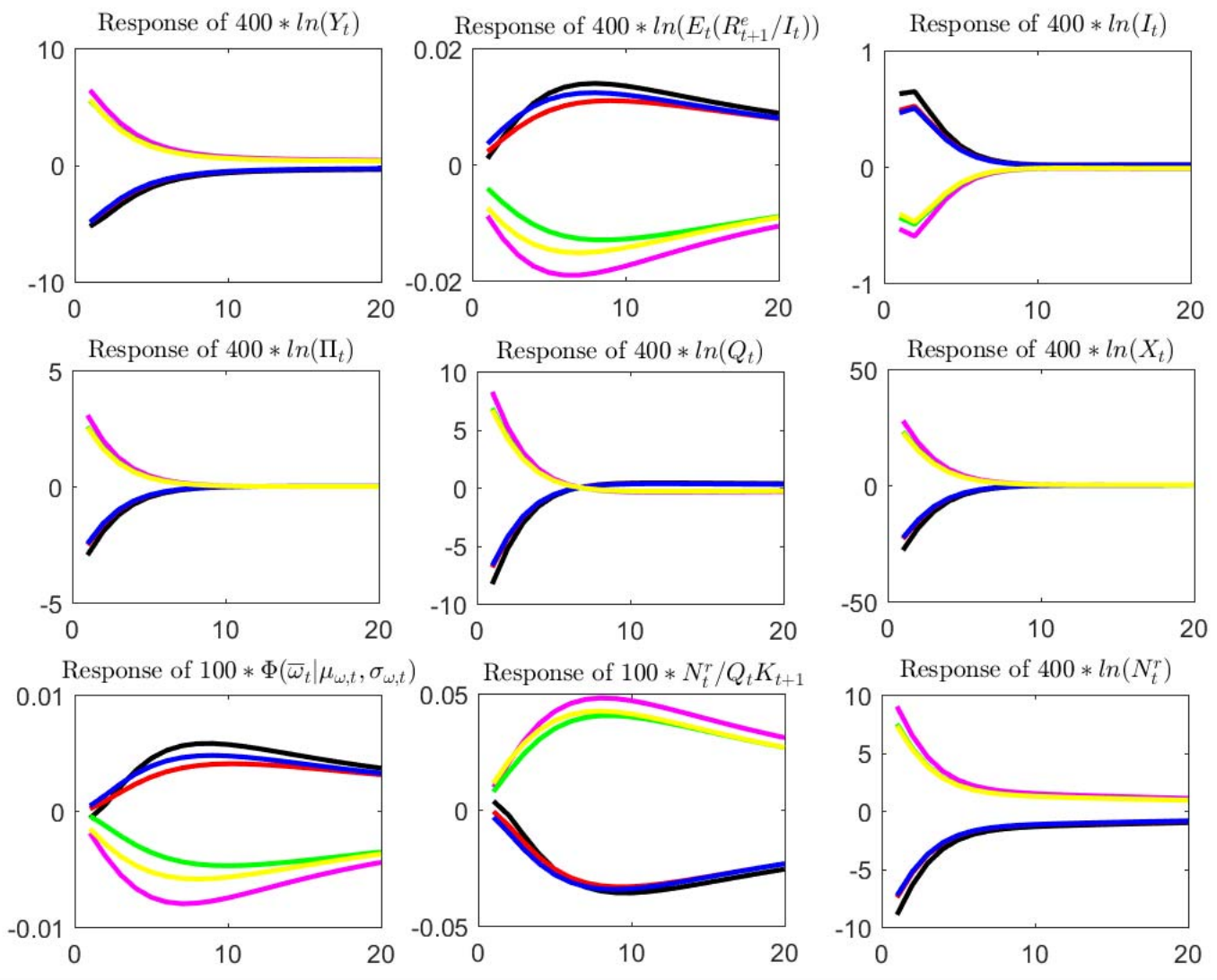

+2 sd - high $\longrightarrow+2$ sd - low -+2 sd - medium $\longrightarrow-2$ sd - high

-2 sd - low

$-2 \mathrm{sd}$ - medium 
Figure 3E. Generalized Impulse Response Functions: Response to a +2/-2 Standard Deviation Interest Rate Rule Stochastic Volatility Shock, Conditional on High, Mid, Low Values of the Endogenous Spread.
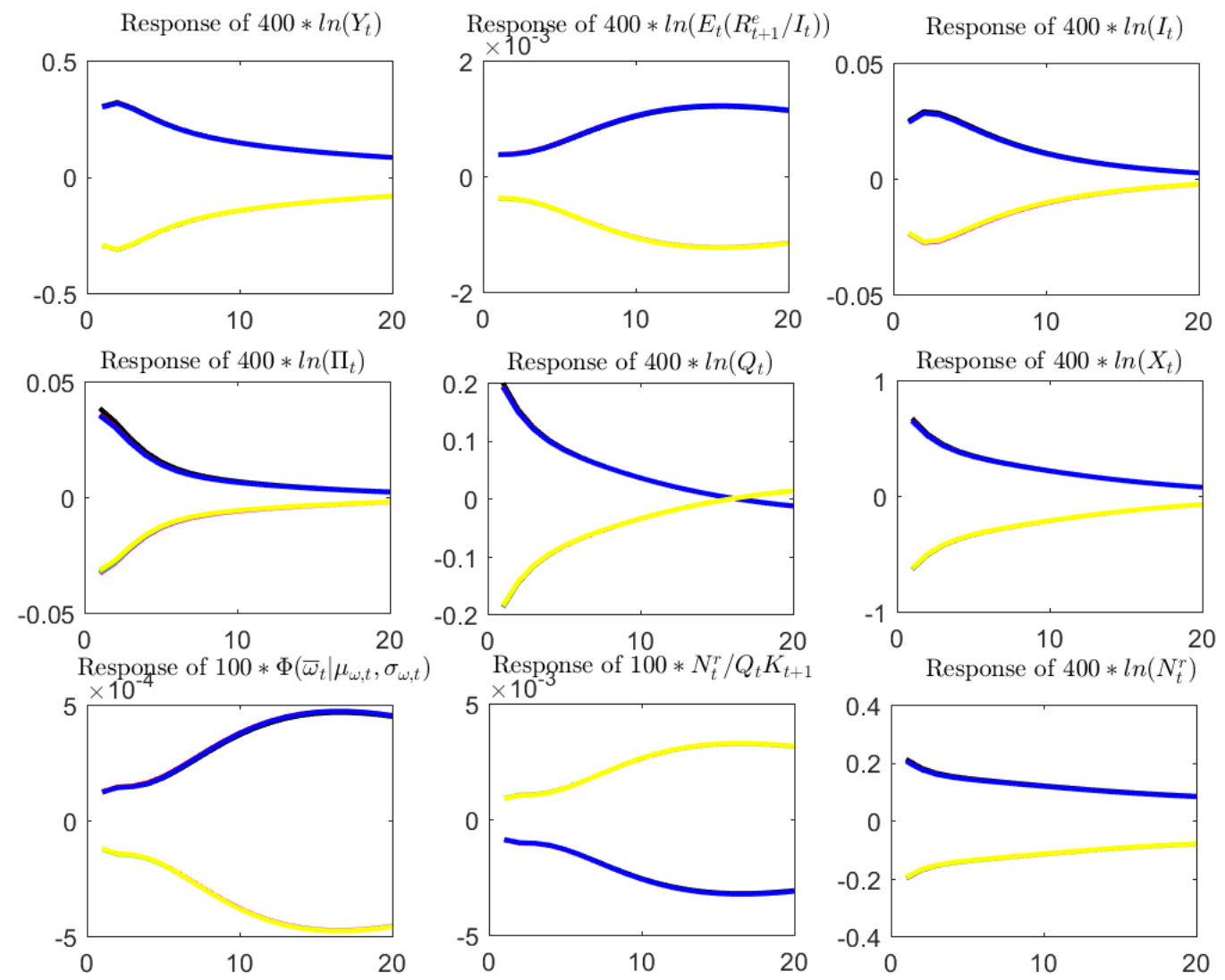
Figure 4A. Generalized Impulse Response Functions: Response to a +1 Standard Deviation TFP Shock, Conditional on the Level of Micro-Uncertainty.
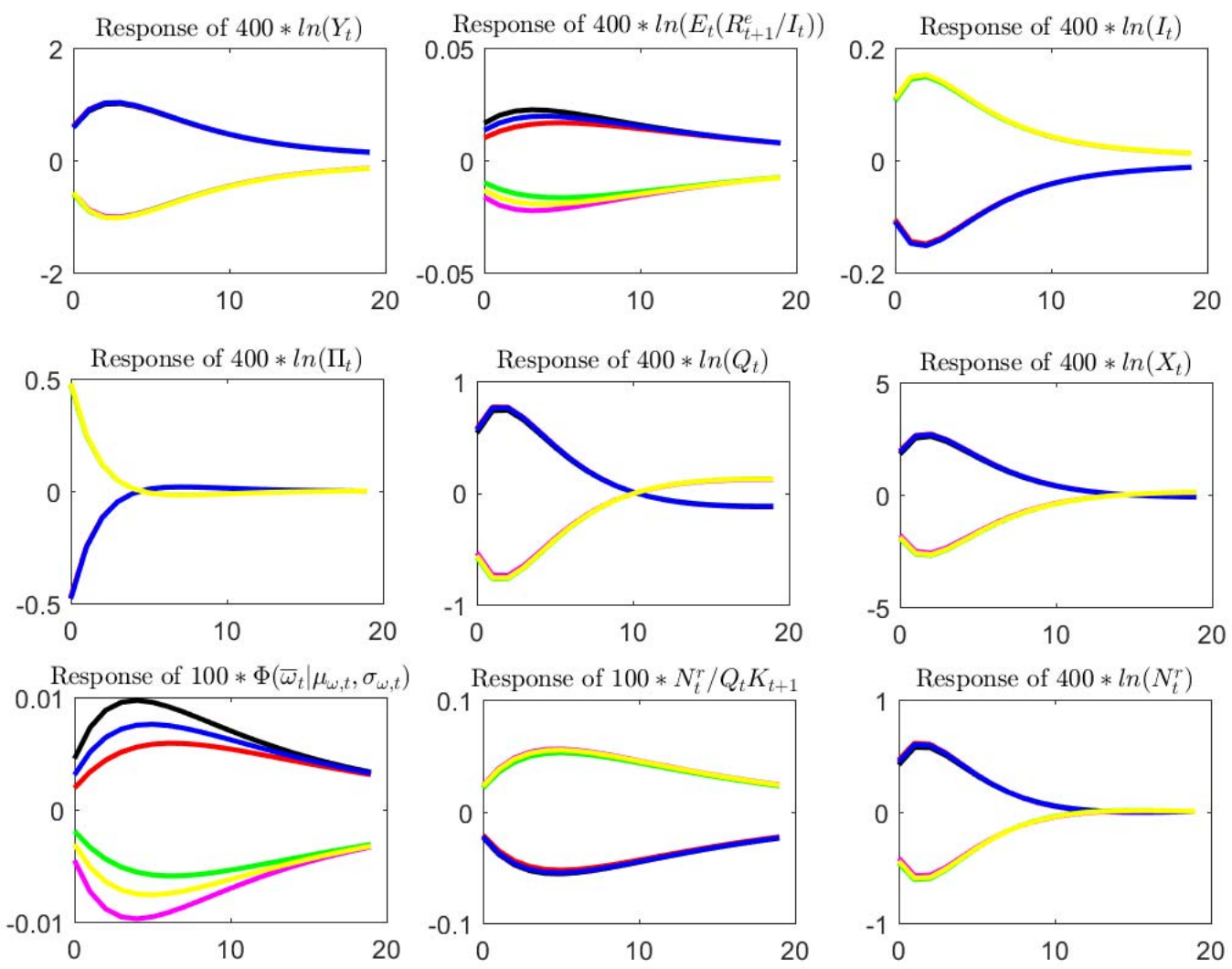

+1 sd - high

+1 sd - low

$+1 \mathrm{sd}-$ medium

-1 sd - high

-1 sd - low

$-1 \mathrm{sd}$ - medium 
Figure 4B. Generalized Impulse Response Functions: Response to a +1 Standard Deviation TFP Stochastic Volatility Shock, Conditional on the Level of Micro-Uncertainty.
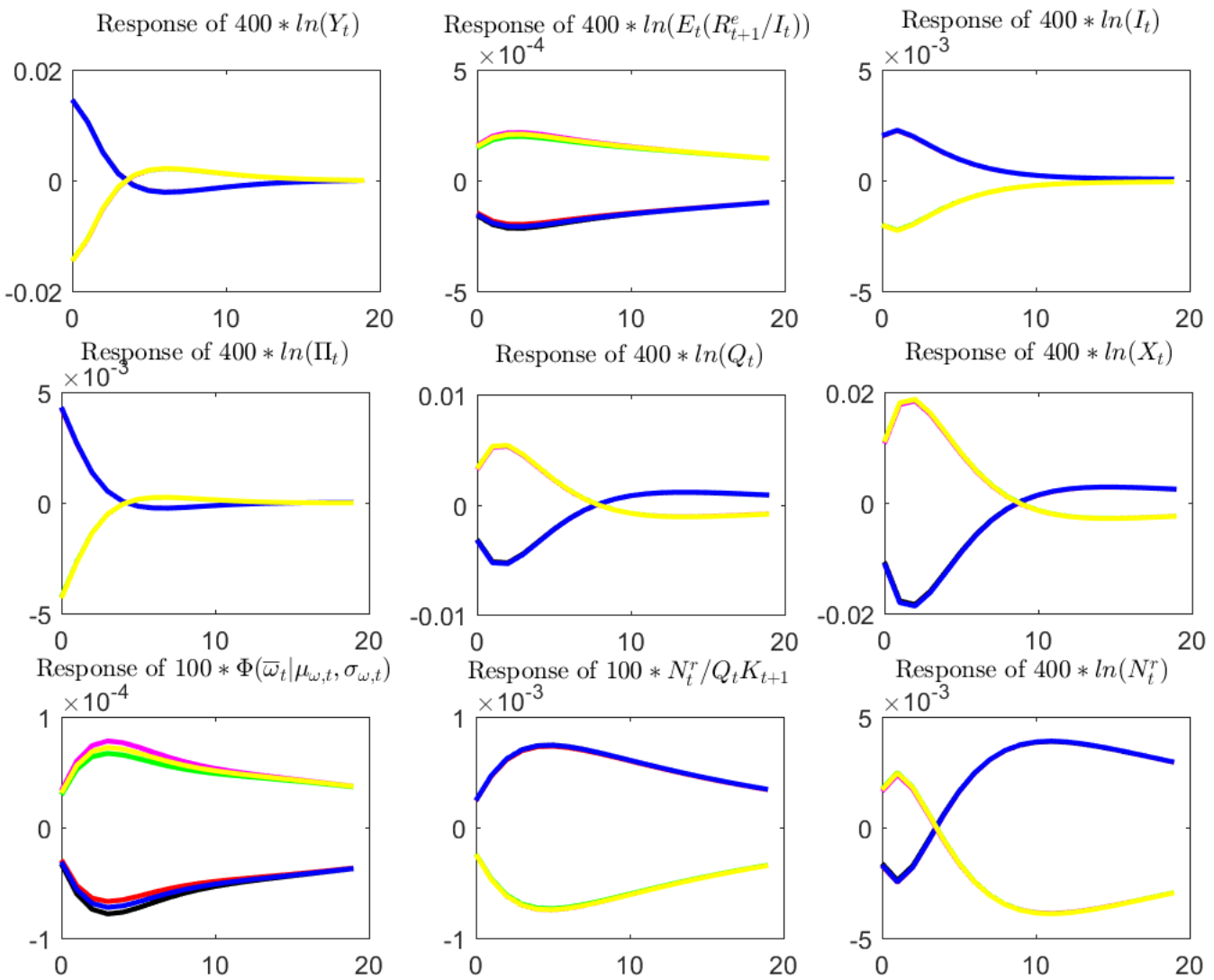

+1 sd - high $\longrightarrow+1$ sd - low

$+1 \mathrm{sd}-$ medium $\longrightarrow-1 \mathrm{sd}-\mathrm{high}$

-1 sd - low

$-1 \mathrm{sd}-$ medium 
Figure 4C. Generalized Impulse Response Functions: Response to a +1 Standard Deviation Micro-Uncertainty Shock, Conditional on the Level of Micro-Uncertainty.
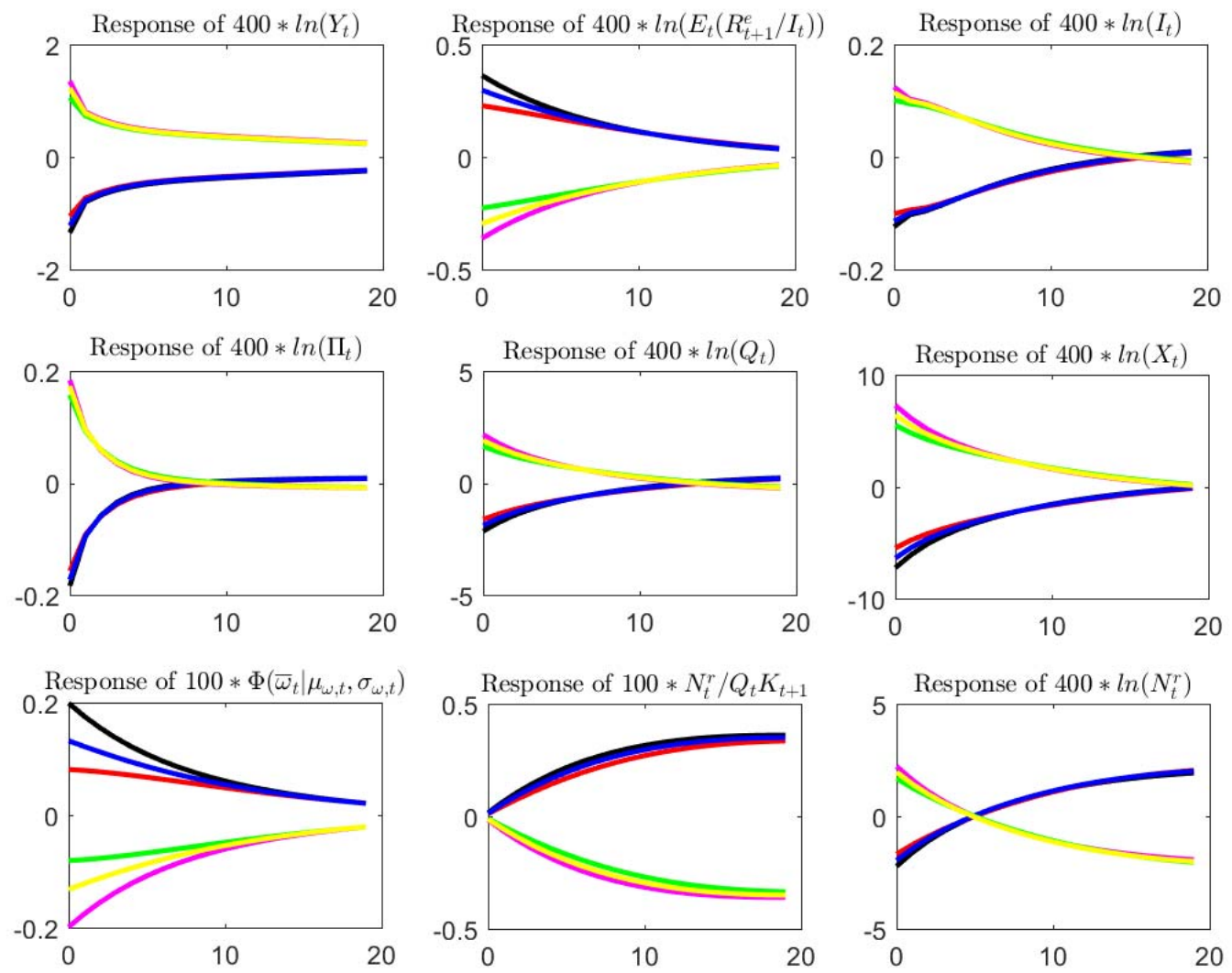
Figure 4D. Generalized Impulse Response Functions: Response to a +1 Standard Deviation Interest Rate Rule Shock, Conditional on the Level of Micro-Uncertainty.
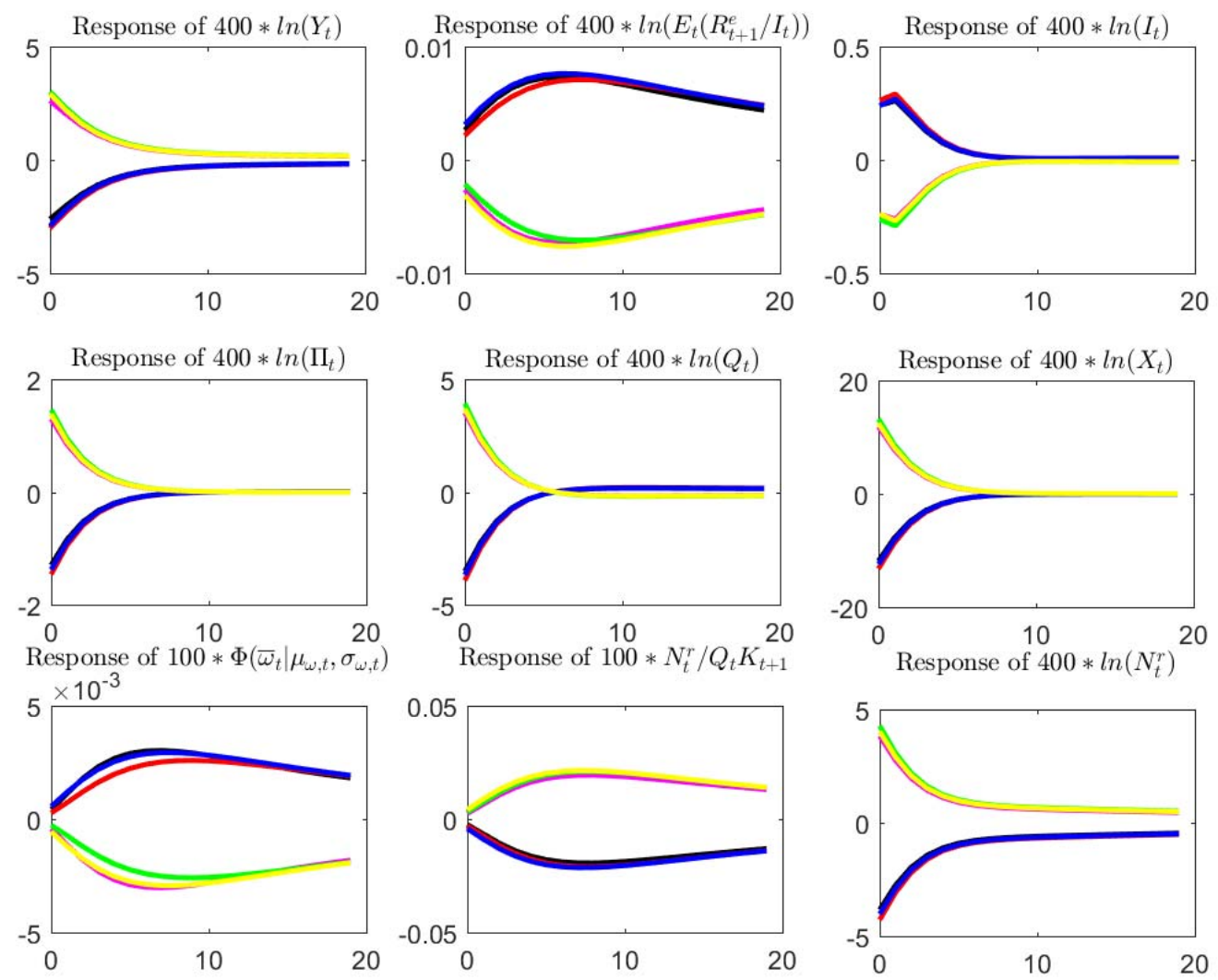

+1 sd - high $\longrightarrow+1$ sd - low

$+1 \mathrm{sd}-$ medium

$-1 \mathrm{sd}-$ high

-1 sd - low

$-1 \mathrm{sd}-$ medium 
Figure 4E. Generalized Impulse Response Functions: Response to a +1 Standard Deviation Interest Rate Rule Stochastic Volatility Shock, Conditional on the Level of Micro-Uncertainty.
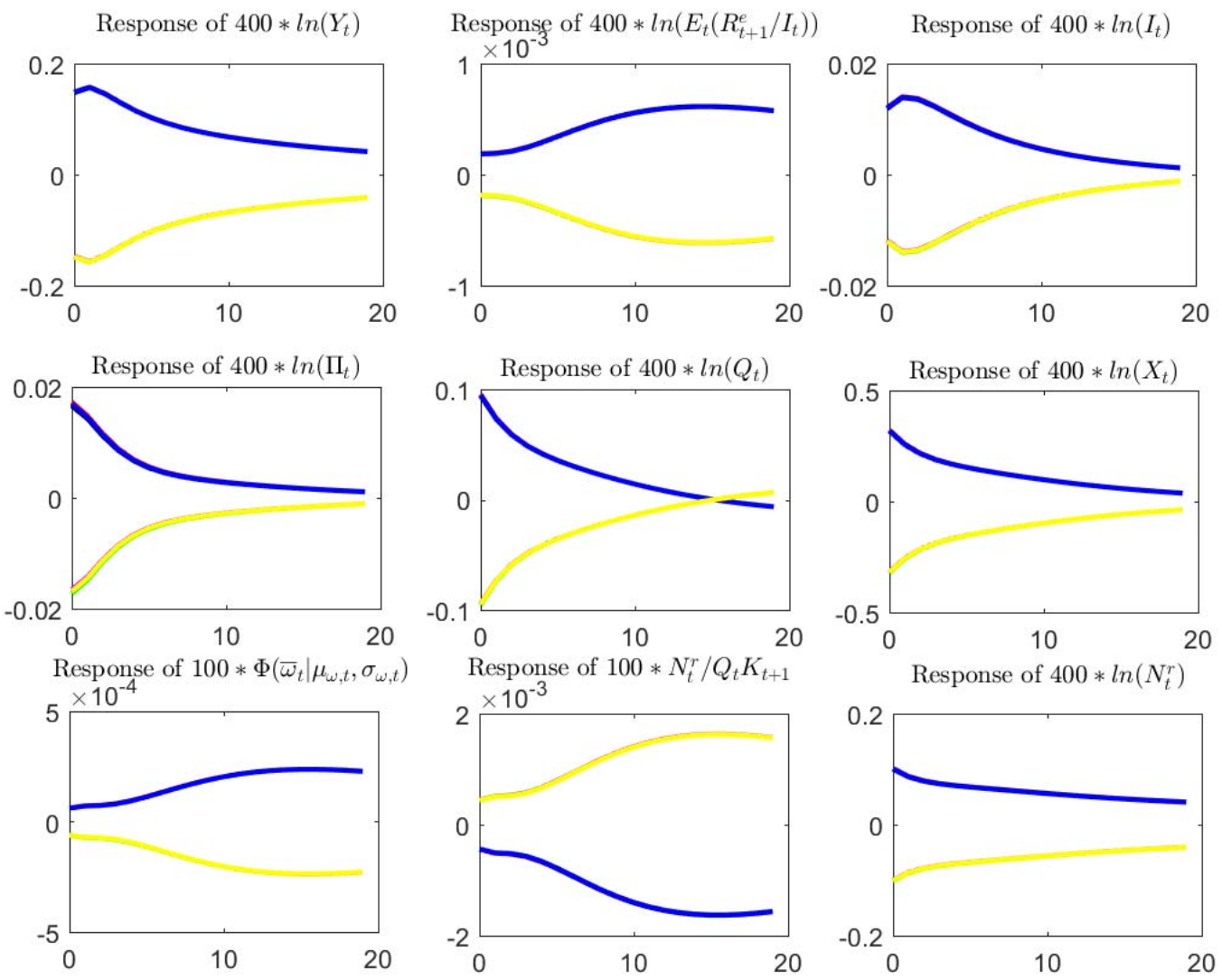
Figure 5A. Generalized Impulse Response Functions: Response to a +1 Shock, Initial Condition: Unconditional Mean.
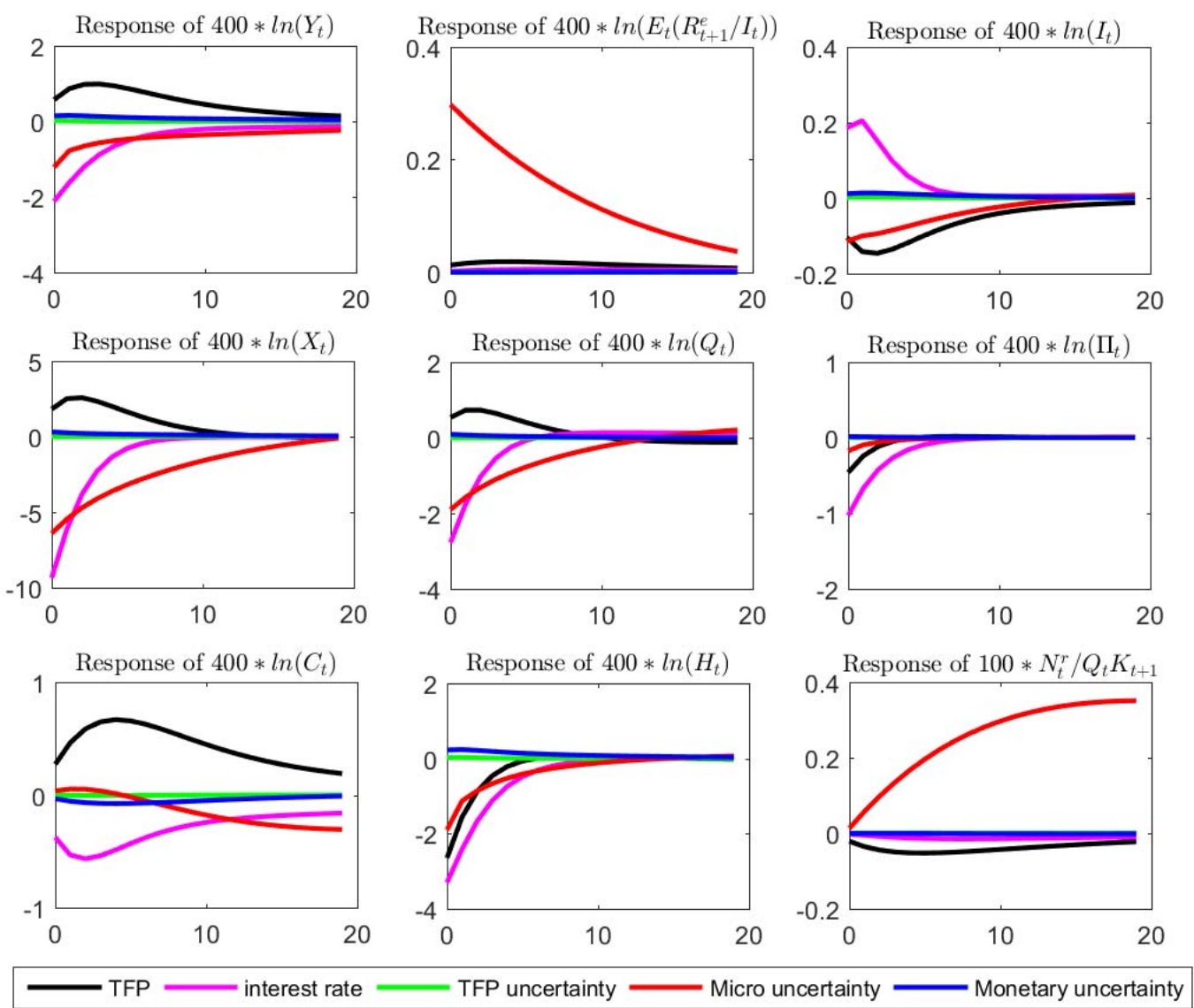
Figure 5B. Generalized Impulse Response Functions: Response to a +1 Shock, Initial Condition: Conditional on 5th and 95th Percentiles on Spread.
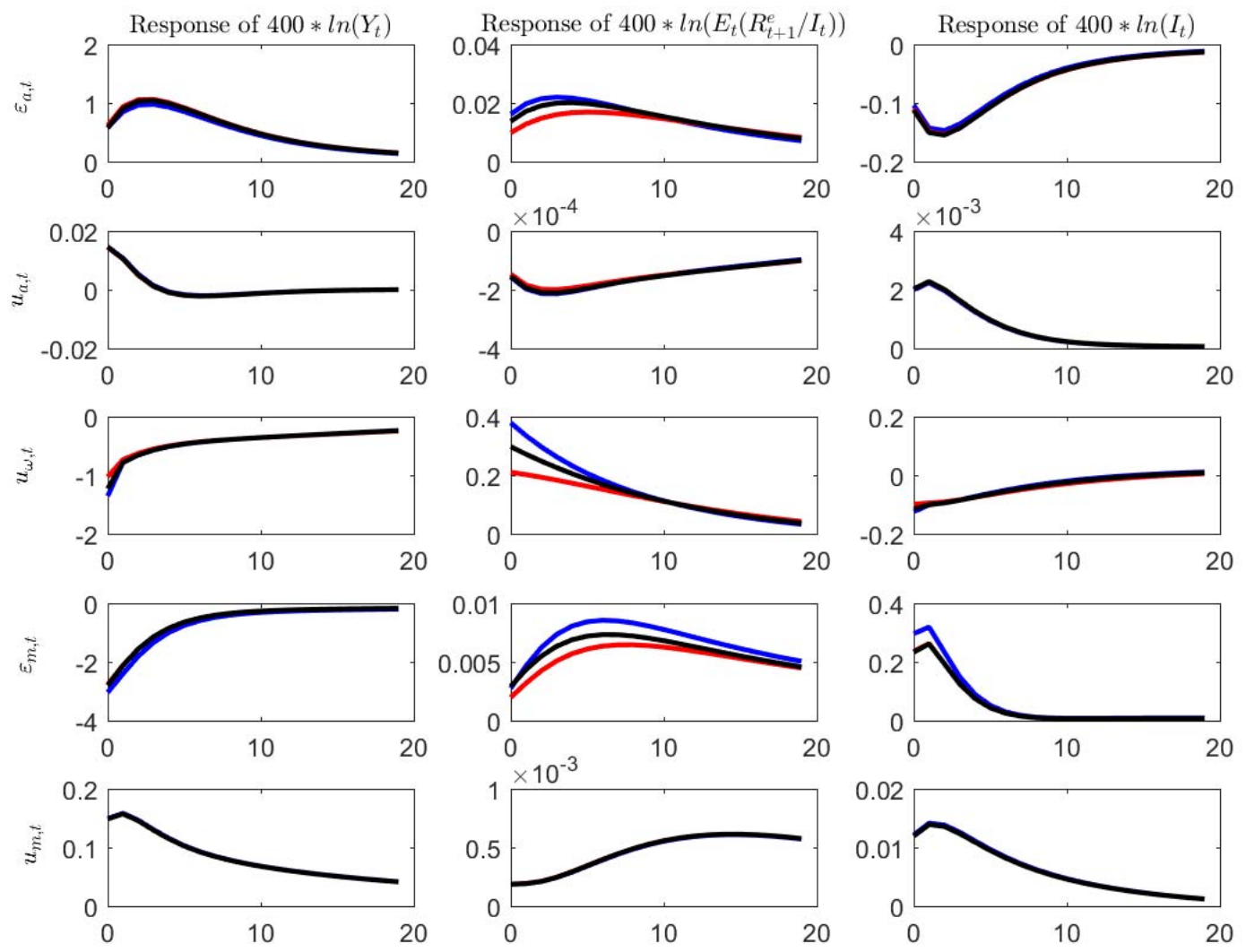

High spread (95th) — Low spread (5th)

- Mid spread (50th) 
Figure 5C. Generalized Impulse Response Functions: Response to a +1 Shock, Initial Condition: Conditional on 1st and 99th Percentiles on Spread.
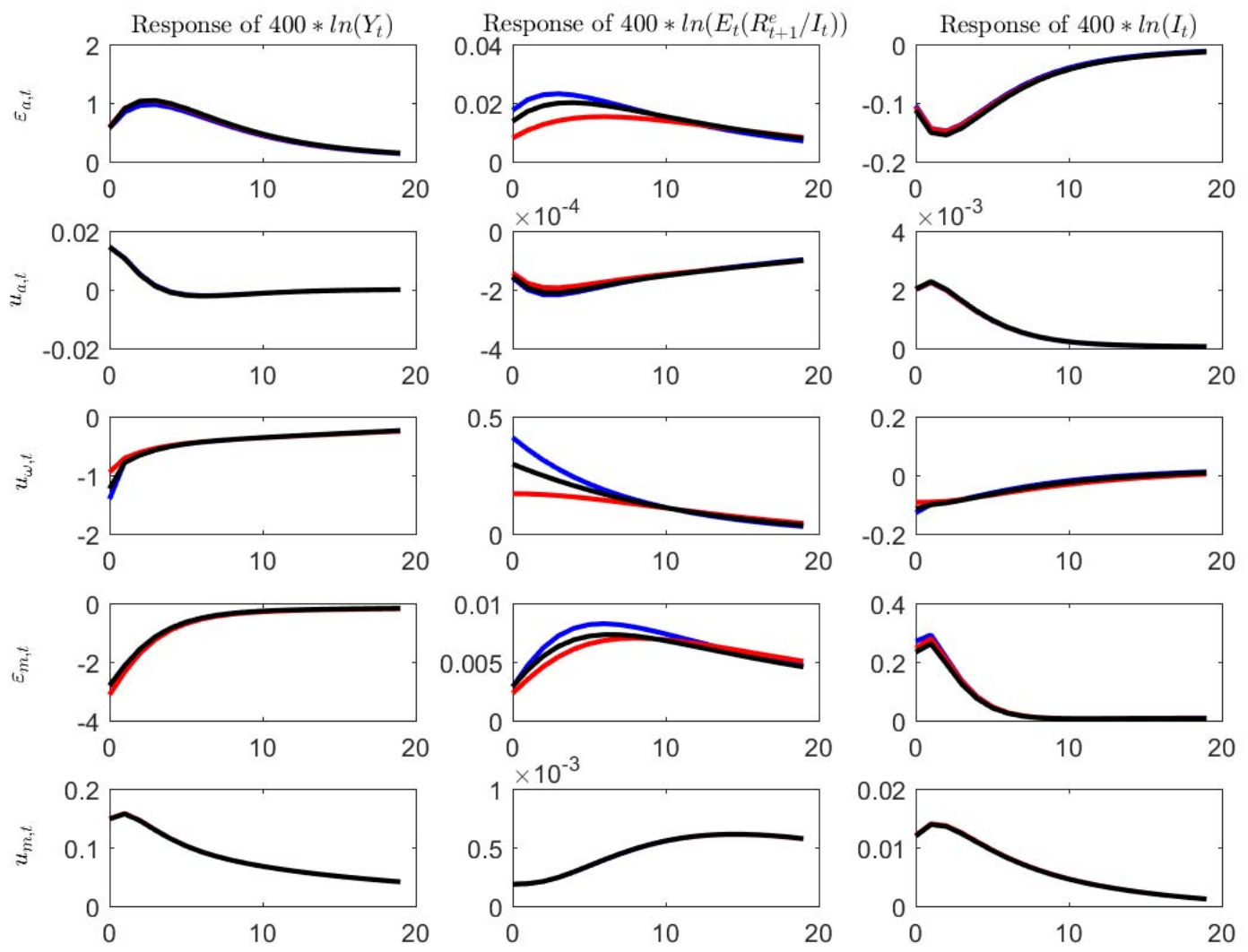

- High spread (99th) — Low spread (1st)

Mid spread (50th) 
Figure 6A. Generalized Impulse Response Functions: Response to a +1 Shock, For Alternative Model Specifications, Conditional on High Spreads.
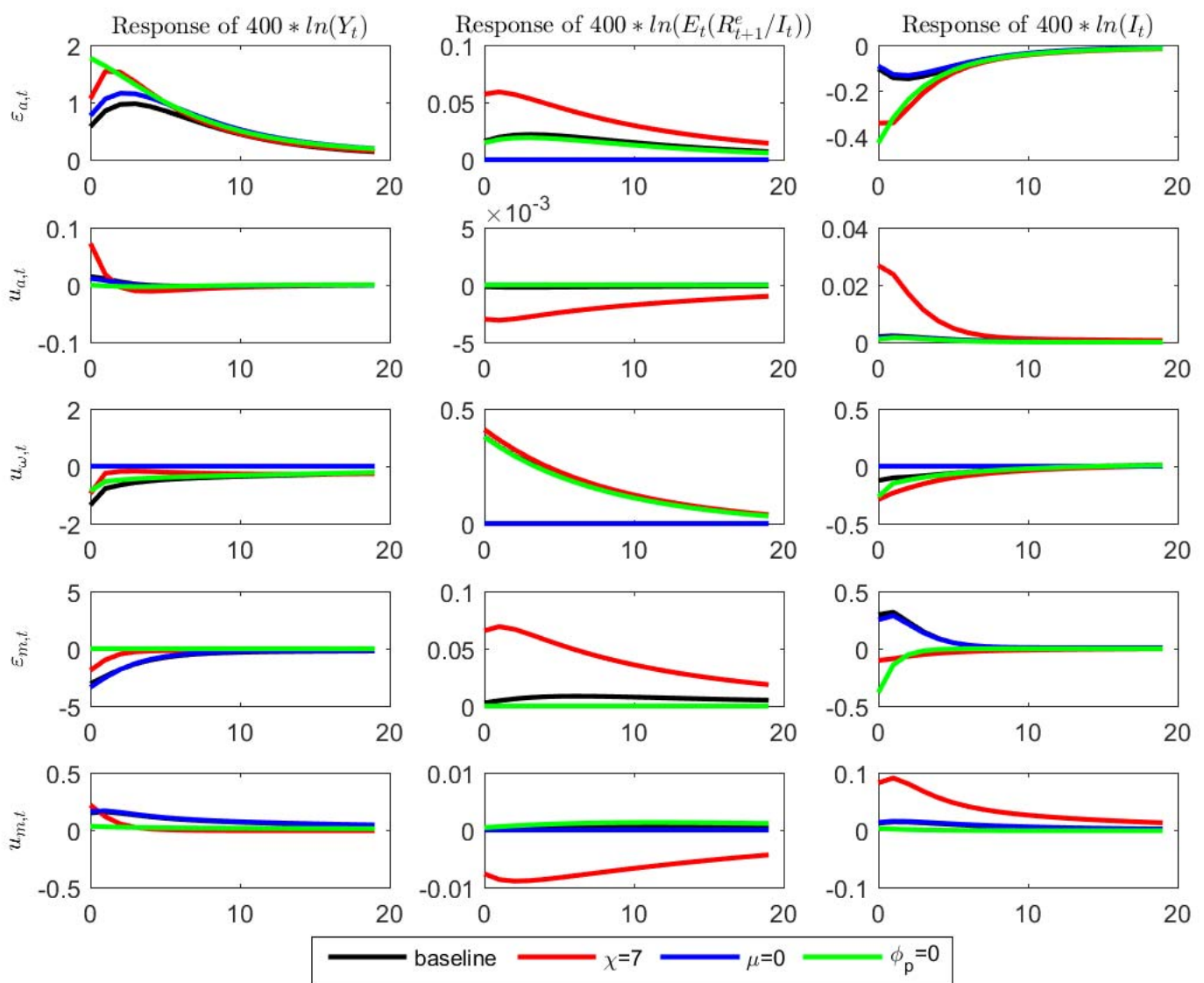
Figure 6B. Generalized Impulse Response Functions: Response to a +1 Shock, Conditional on High and Low Spreads, With and Without Micro-Uncertainty.
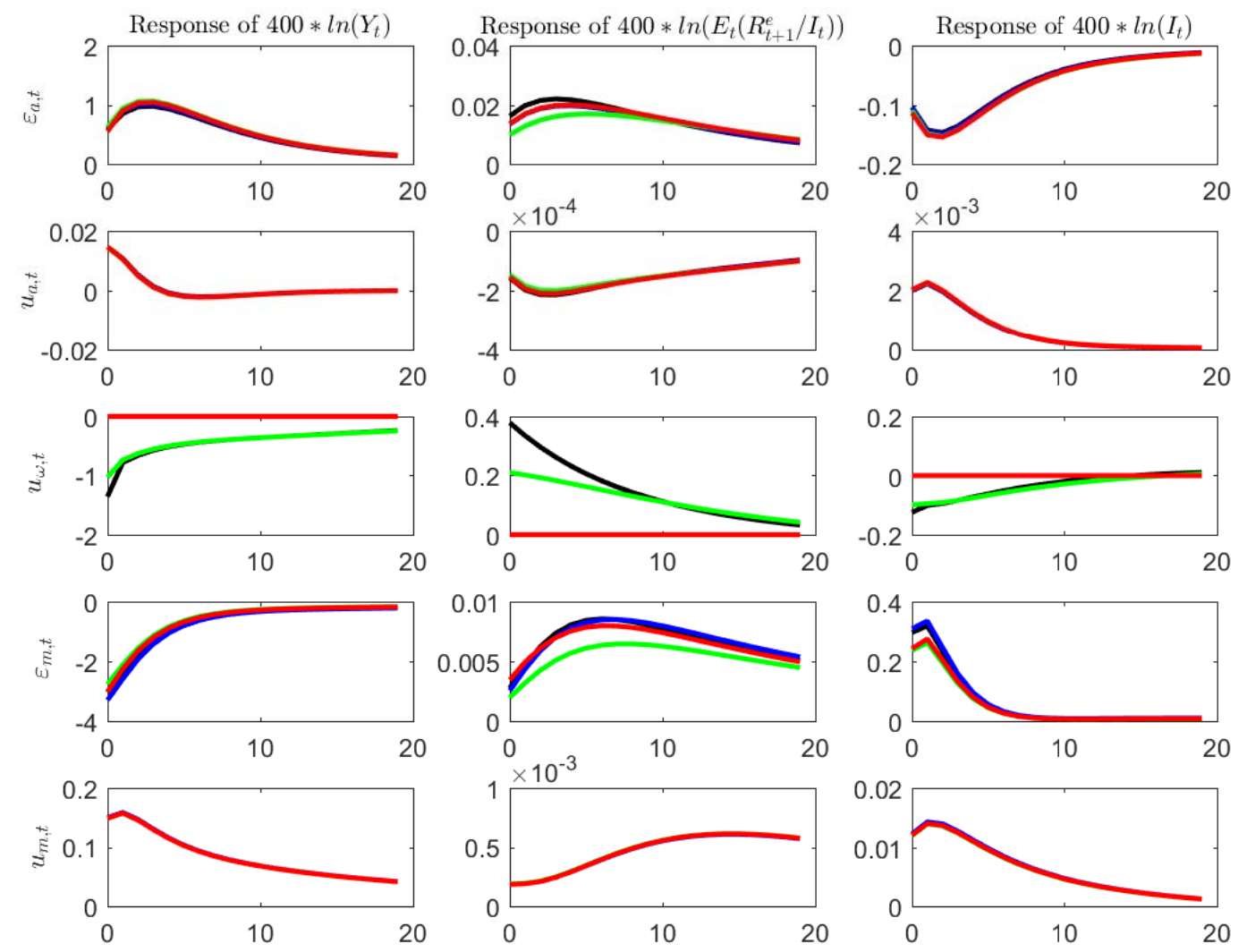

High spread $\longrightarrow$ High spread, no micro uncert. $\quad$ Low spread $\quad$ Low spread, no micro uncert. 
Figure 6C. Generalized Impulse Response Functions: Response to a +1 Shock, Conditional on High Uncertainty (Individually).
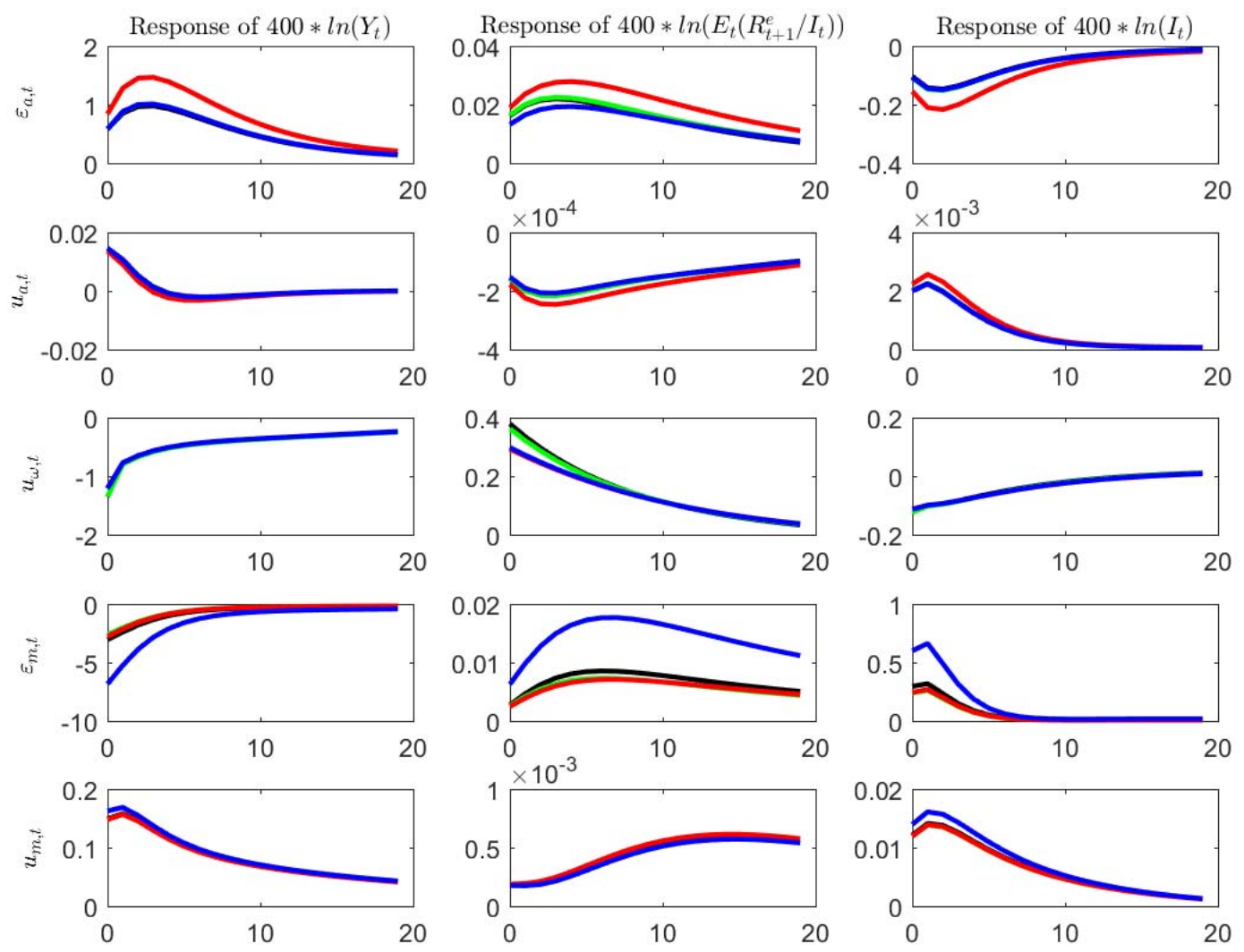

- High Spread — High micro uncertainty — High TFP uncertainty — High monetary uncertainty 
Figure 6D. Generalized Impulse Response Functions: Response to a +1 Shock, When All Three Stochastic Volatility Shocks are High or Low Simultaneously ( $>$ 80th Percentile or $<$ 20th Percentile).
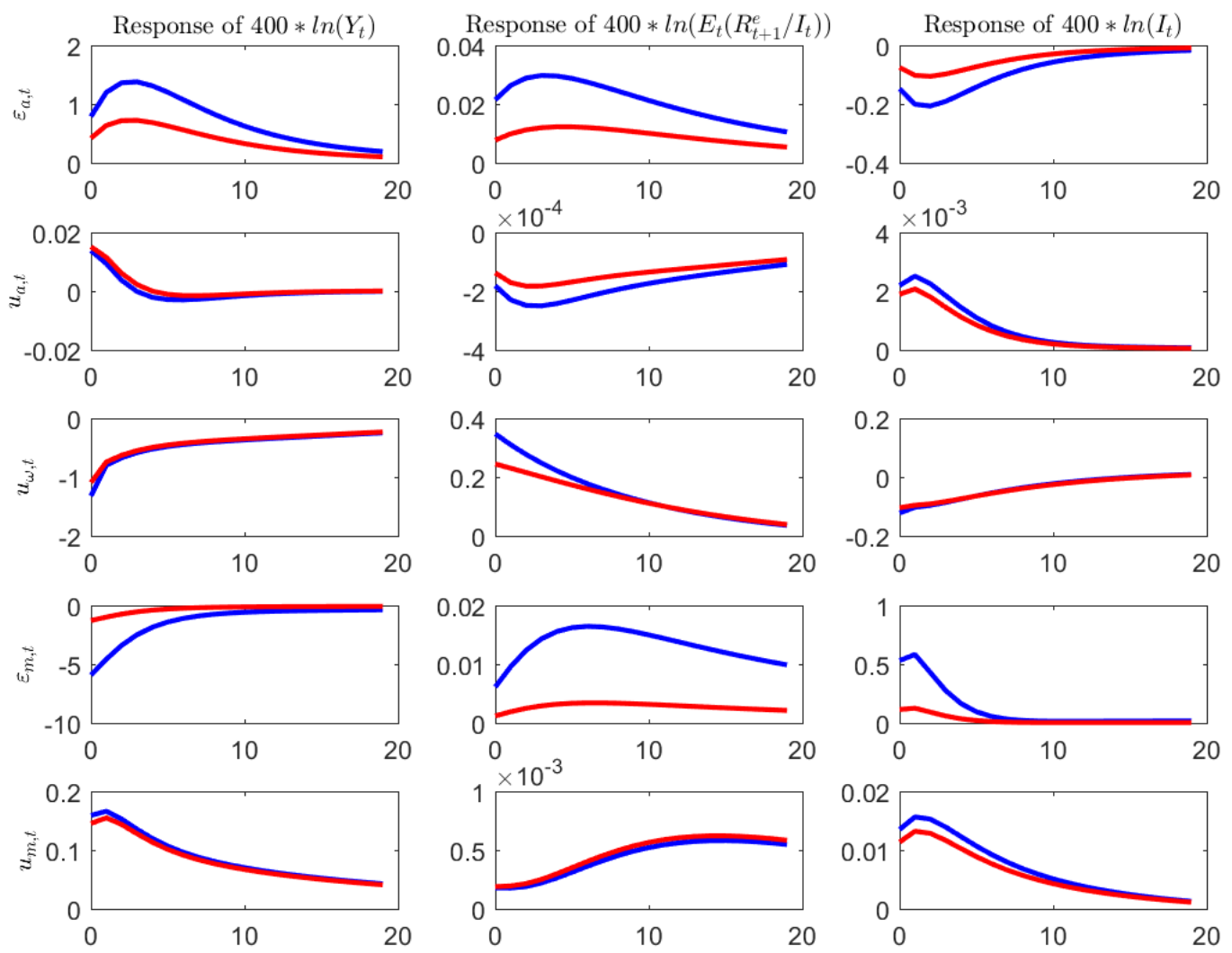

High uncedrtainty $\longrightarrow$ Low uncertainty 
Figure 7A. Generalized Impulse Response Functions: Response to a +1 Standard Deviation TFP Shock, Various Model Specifications, Conditional on Mid-Values of the Endogenous Spread.
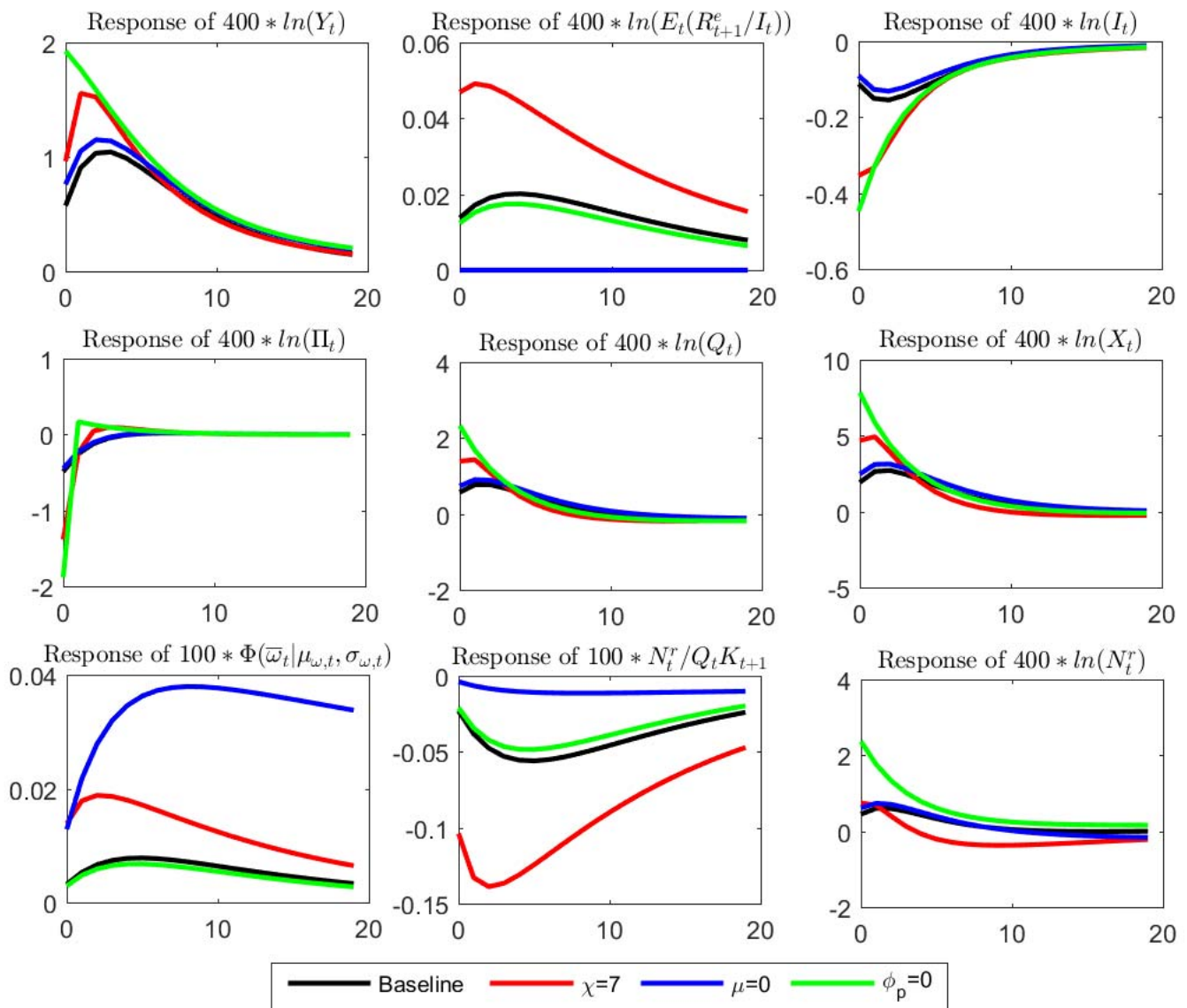
Figure 7B. Generalized Impulse Response Functions: Response to a +1 Standard Deviation TFP Stochastic VolatilityShock, Various Model Specifications, Conditional on Mid-Values of the Endogenous Spread.
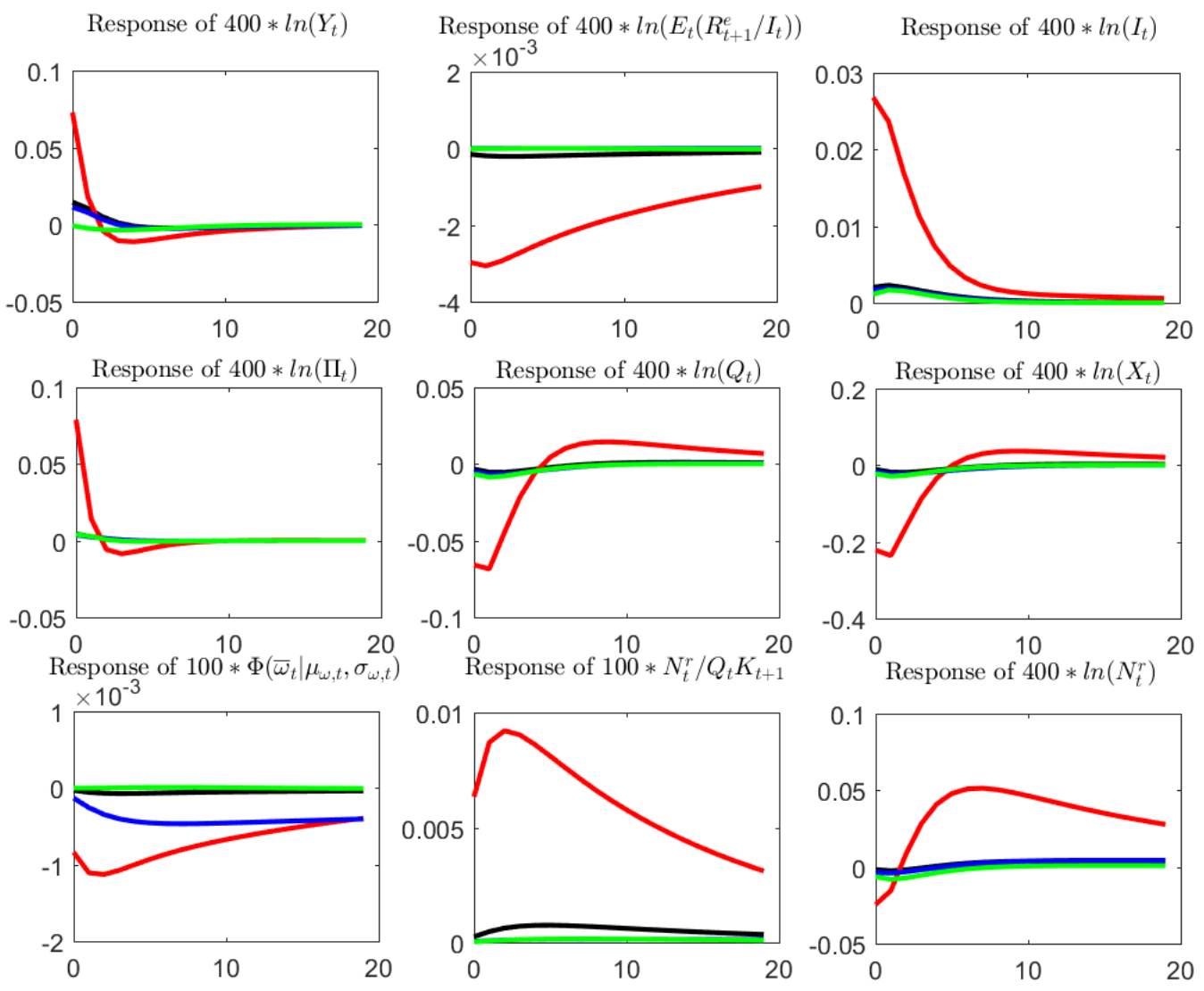
Figure 7C. Generalized Impulse Response Functions: Response to a +1 Standard Deviation Micro-Uncertainty Shock, Various Model Specifications, Conditional on Mid-Values of the Endogenous Spread.
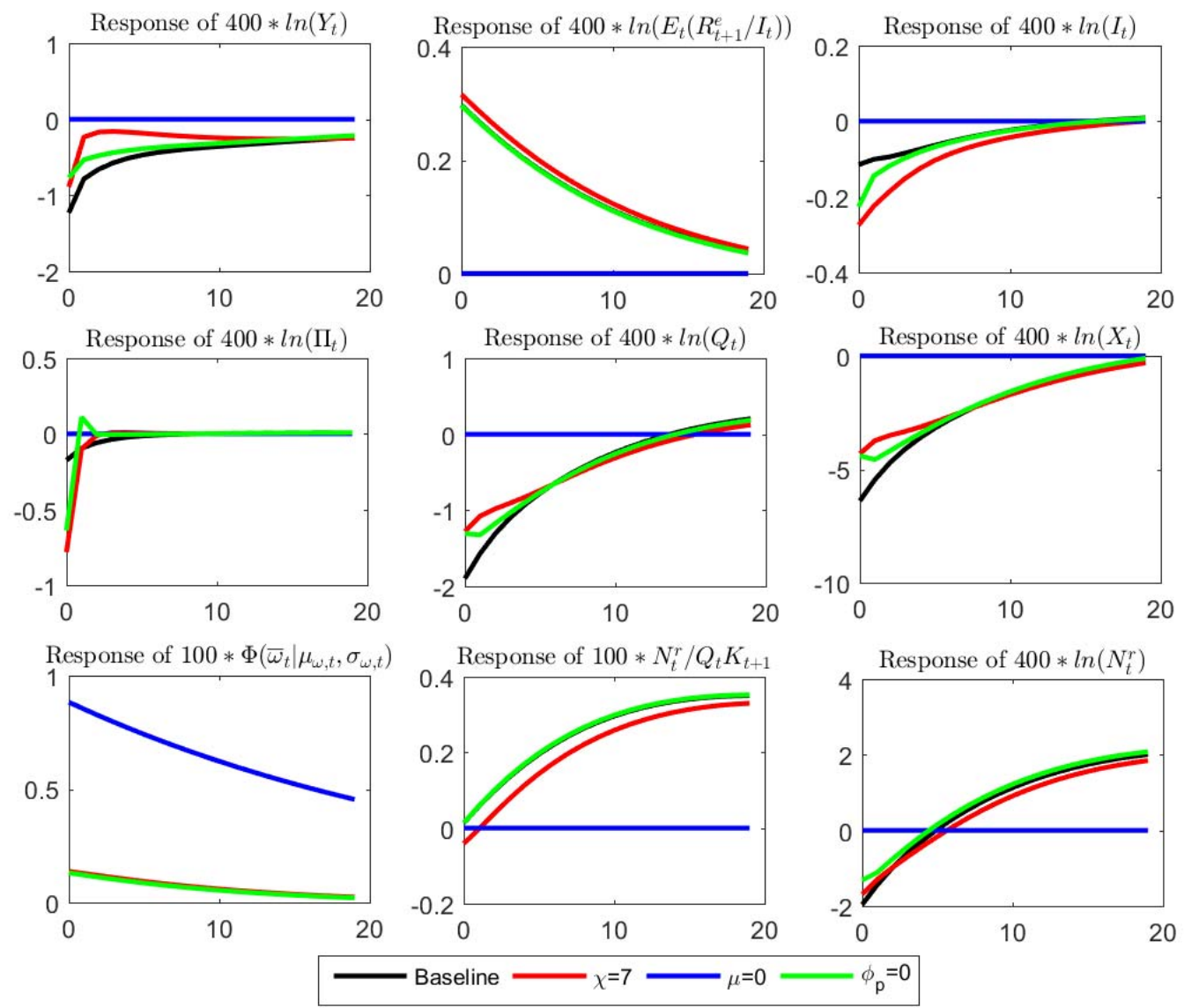
Figure 7D. Generalized Impulse Response Functions: Response to a +1 Standard Deviation Interest Rate Rule Shock, Various Model Specifications, Conditional on Mid-Values of the Endogenous Spread.
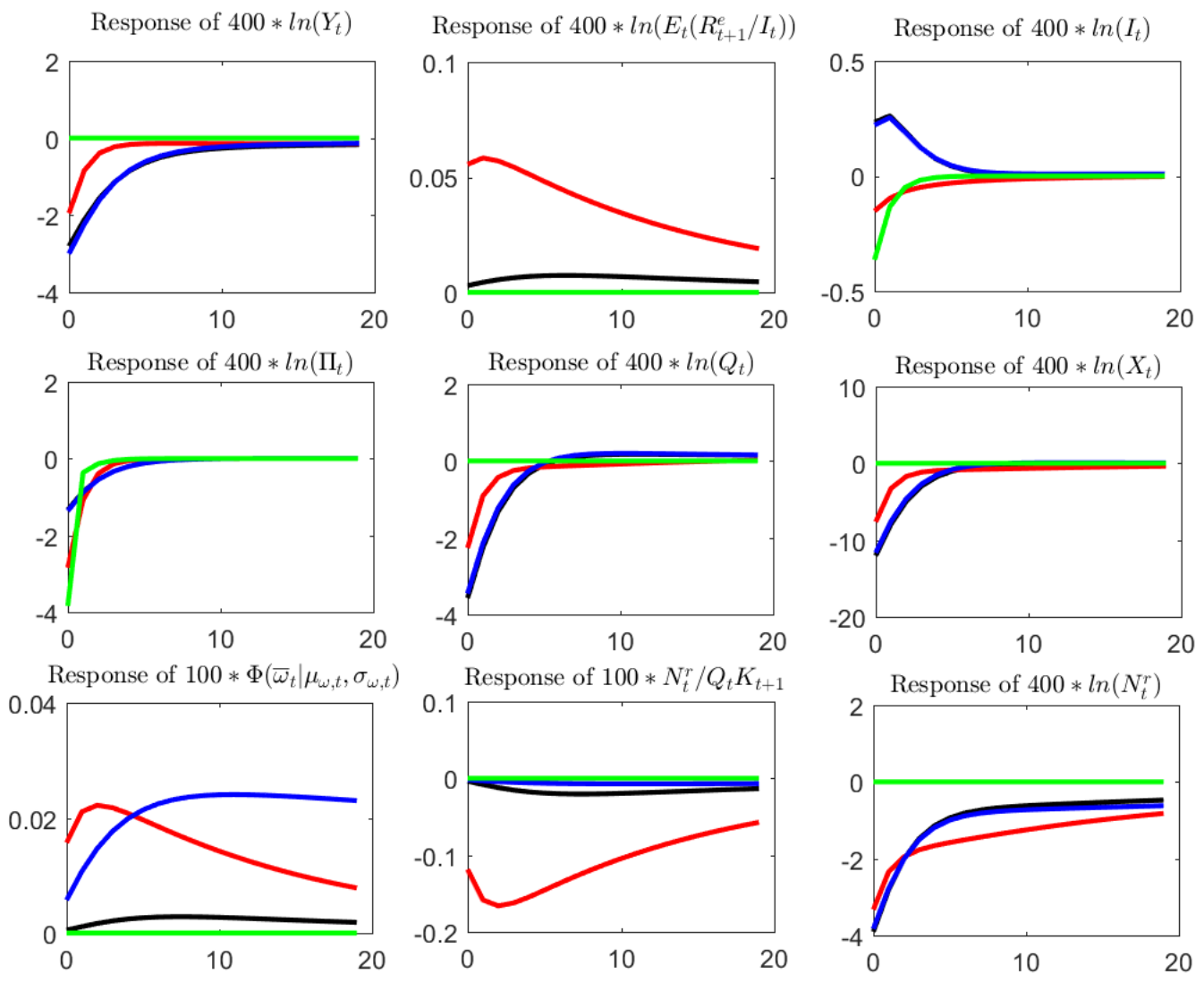
Figure 7E. Generalized Impulse Response Functions: Response to a +1 Standard Deviation Interest Rate Stochastic Volatility Shock, Various Model Specifications, Conditional on Mid-Values of the Endogenous Spread.
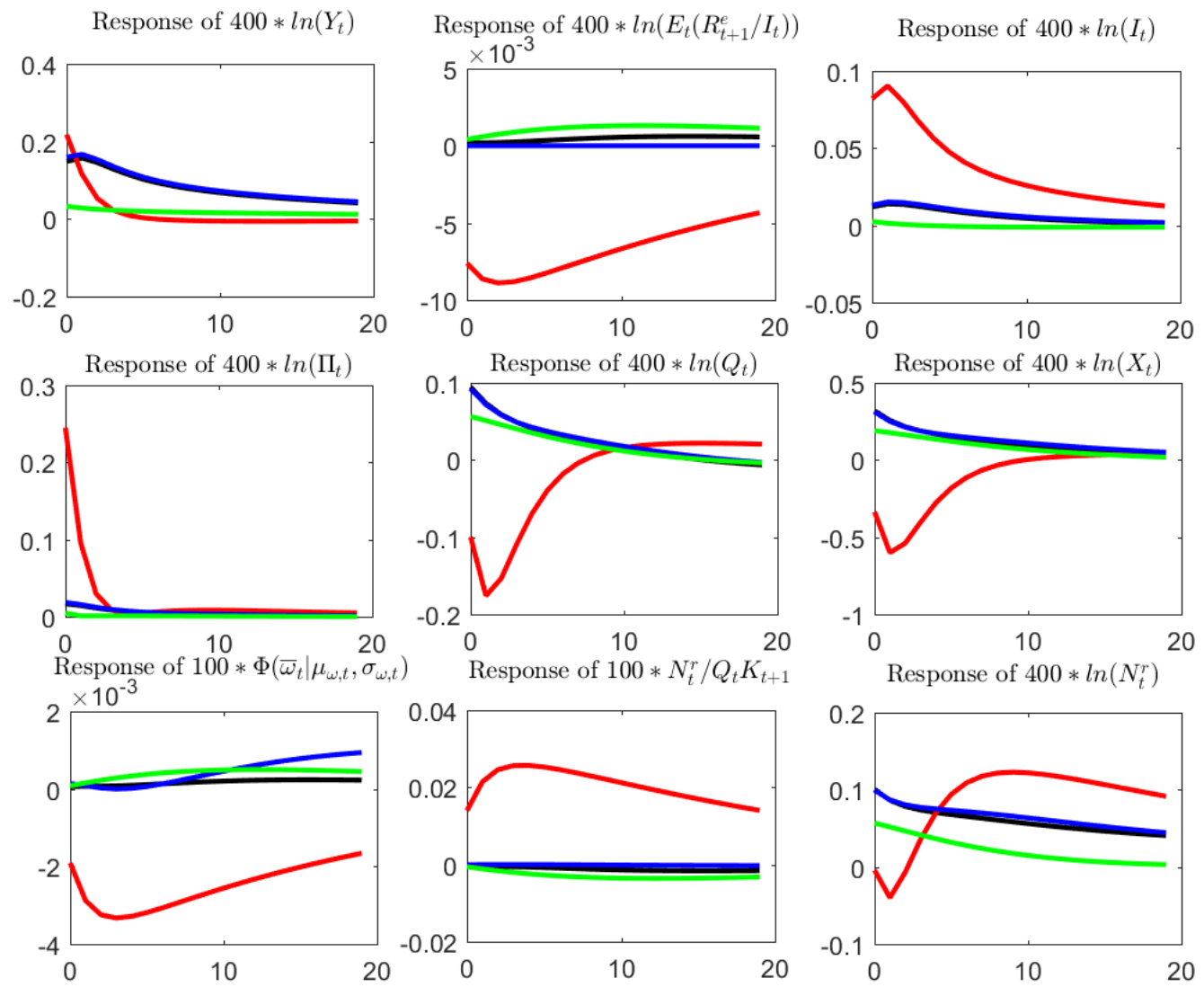

Baseline $\longrightarrow \chi=7-\phi_{\mathrm{p}}=0$ 
Figure 8A. Generalized Impulse Response Functions: Response to a +1 Standard Deviation TFP Shock, Various Model Specifications, Conditional on High-Values of the Endogenous Spread.
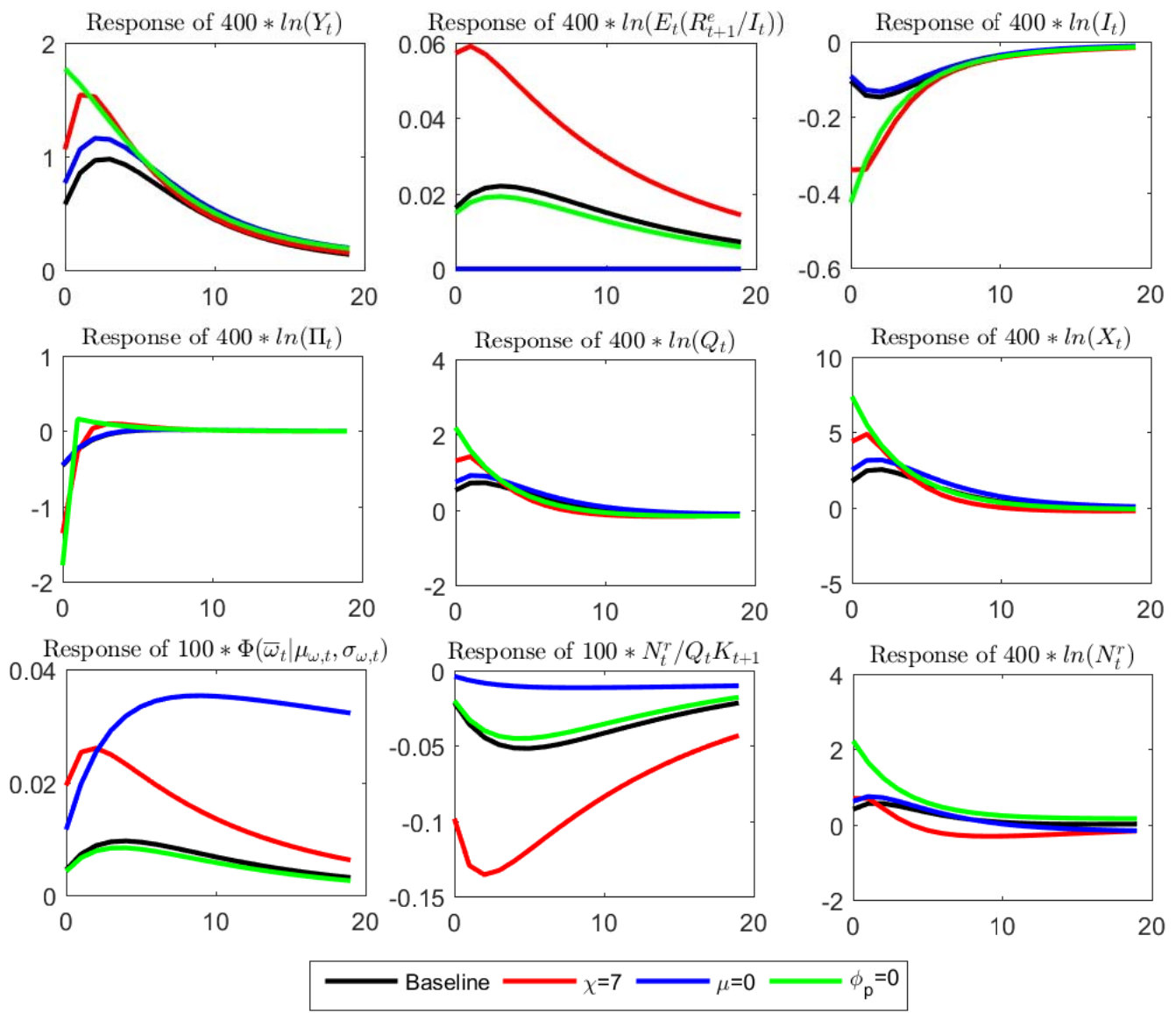
Figure 8B. Generalized Impulse Response Functions: Response to a +1 Standard Deviation TFP Stochastic VolatilityShock, Various Model Specifications, Conditional on Hih-Values of the Endogenous Spread.
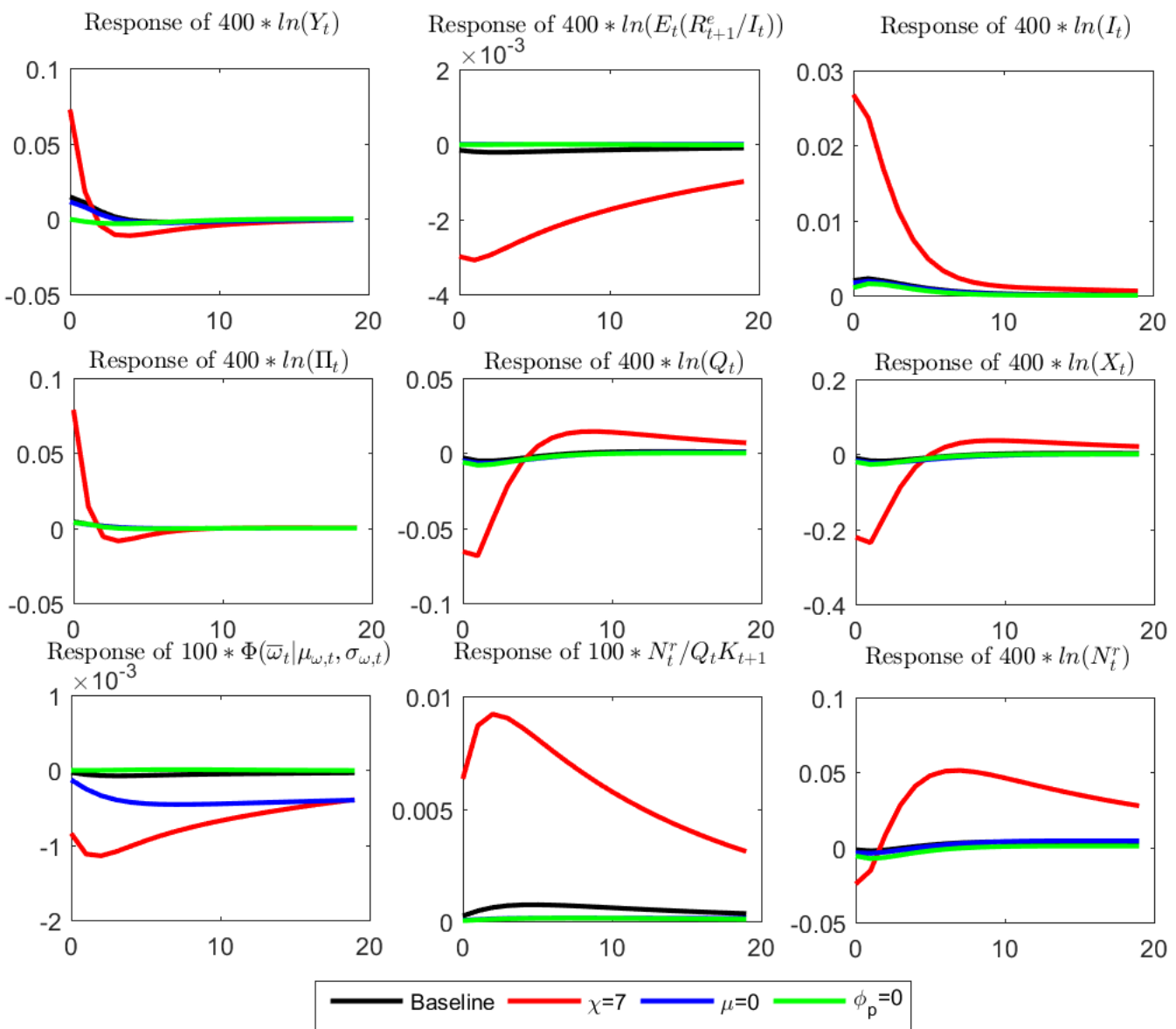
Figure 8C. Generalized Impulse Response Functions: Response to a +1 Standard Deviation Micro-Uncertainty Shock, Various Model Specifications, Conditional on High-Values of the Endogenous Spread.
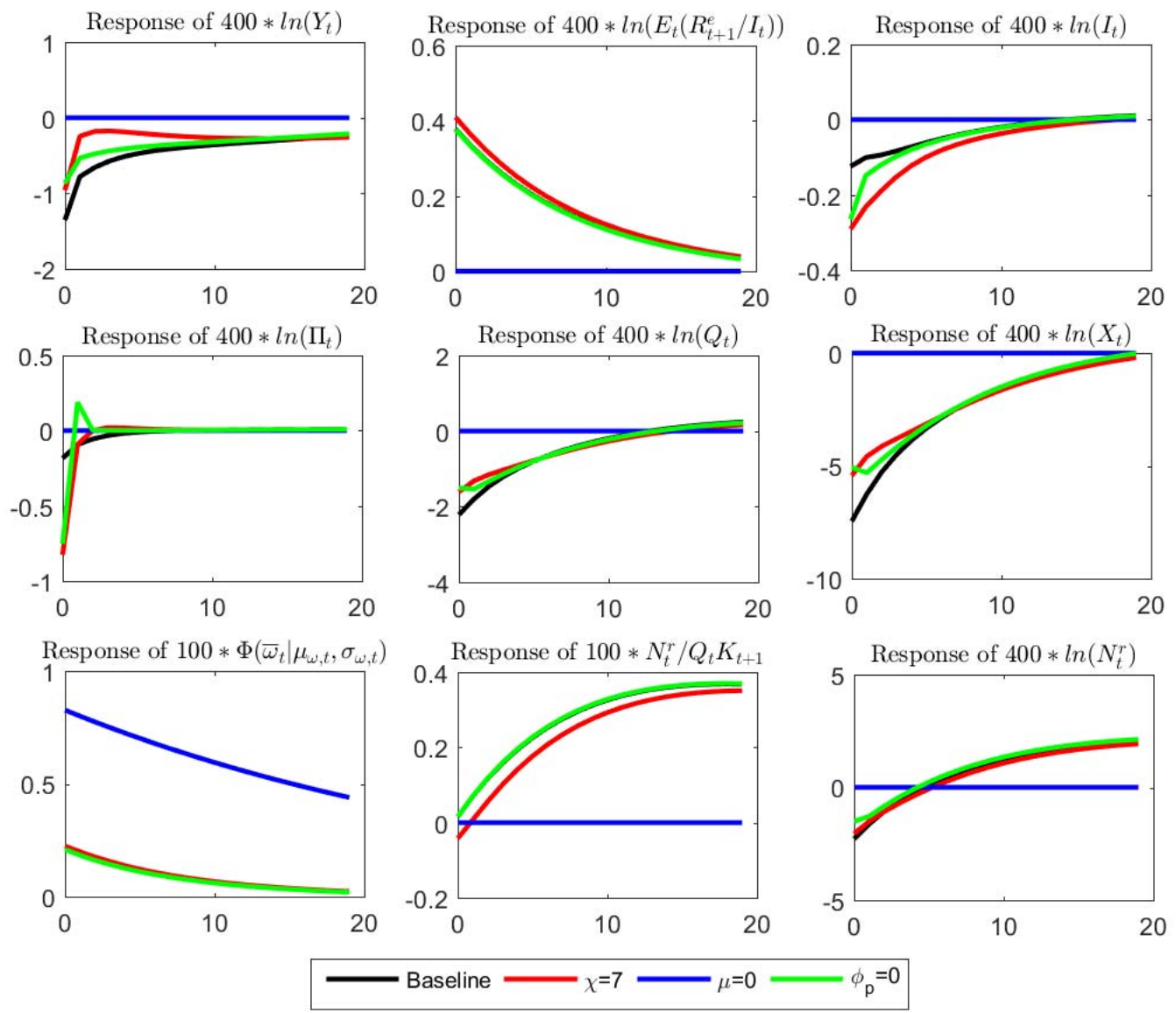
Figure 8D. Generalized Impulse Response Functions: Response to a +1 Standard Deviation Interest Rate Rule Shock, Various Model Specifications, Conditional on High-Values of the Endogenous Spread.
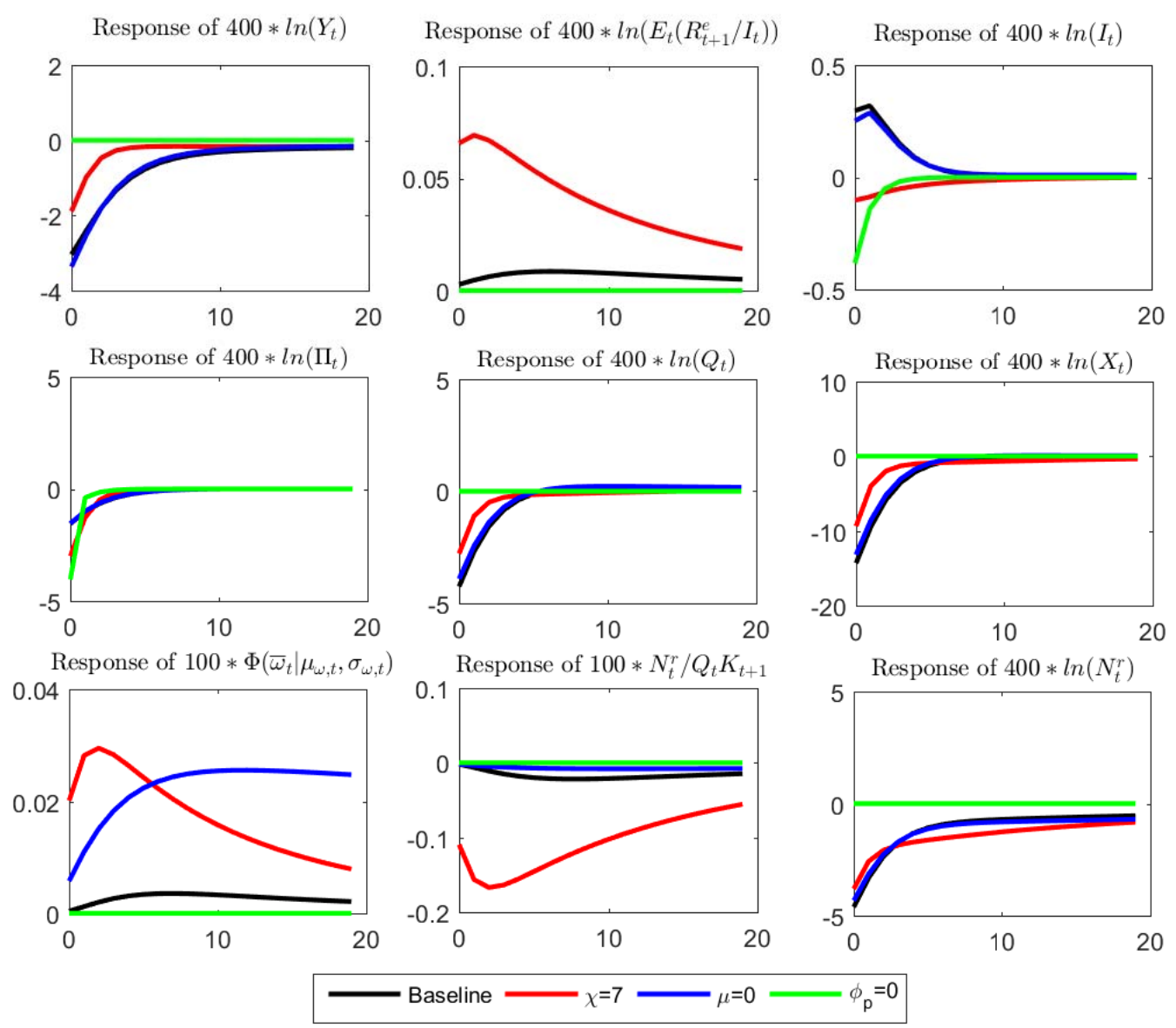
Figure 8E. Generalized Impulse Response Functions: Response to a +1 Standard Deviation Interest Rate Stochastic Volatility Shock, Various Model Specifications, Conditional on High-Values of the Endogenous Spread.
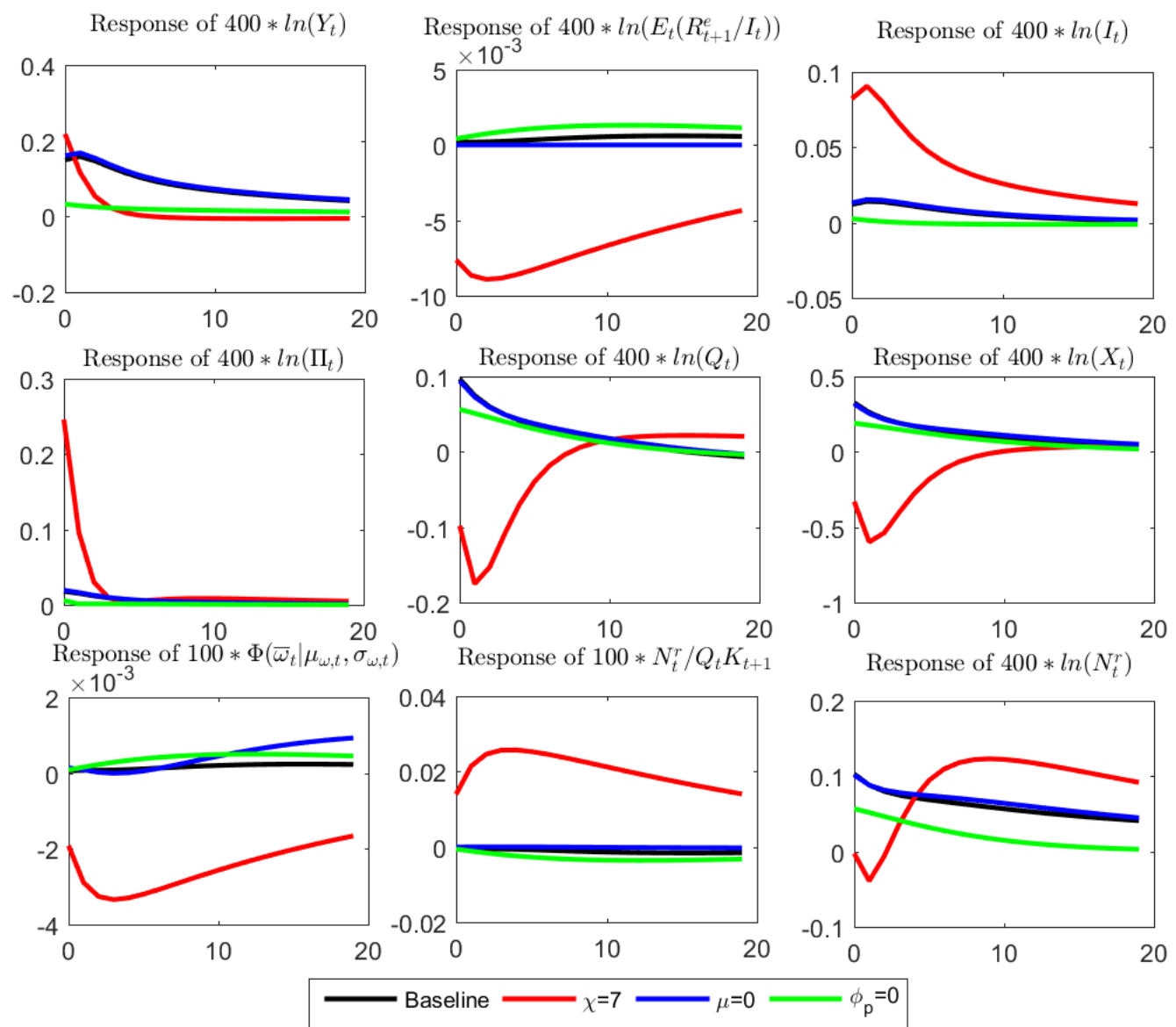
Figure 9A. Generalized Impulse Response Functions: Response to a +1 Standard Deviation TFP Shock, Conditional on High-Values of Spread, Interest Rate, and Leverage Ratio.
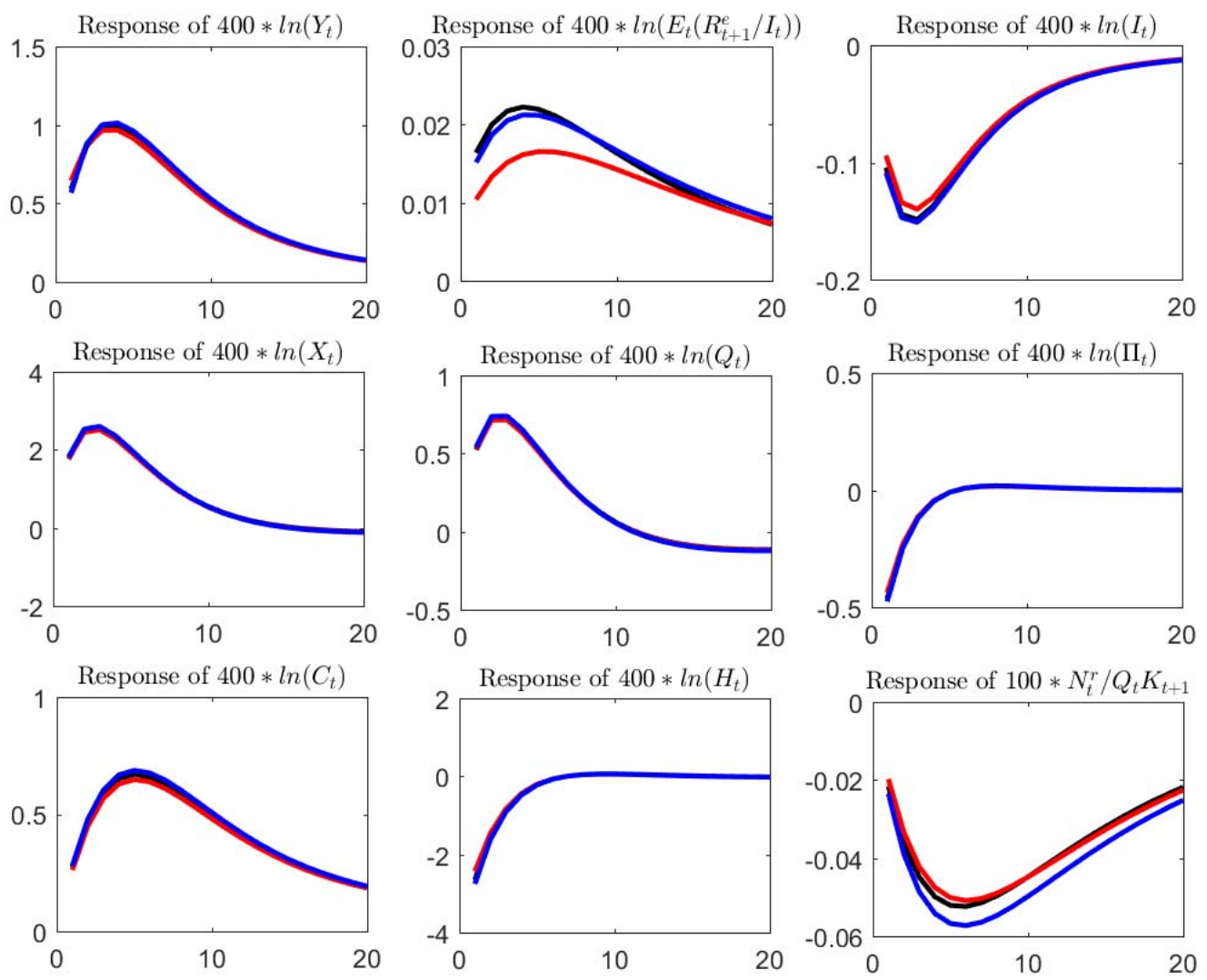

high spread $\longrightarrow$ high interest rate $\longrightarrow$ high leverage 
Figure 9B. Generalized Impulse Response Functions: Response to a +1 Standard Deviation TFP Stochastic Volatility Shock, Conditional on High-Values of Spread, Interest Rate, and Leverage Ratio.
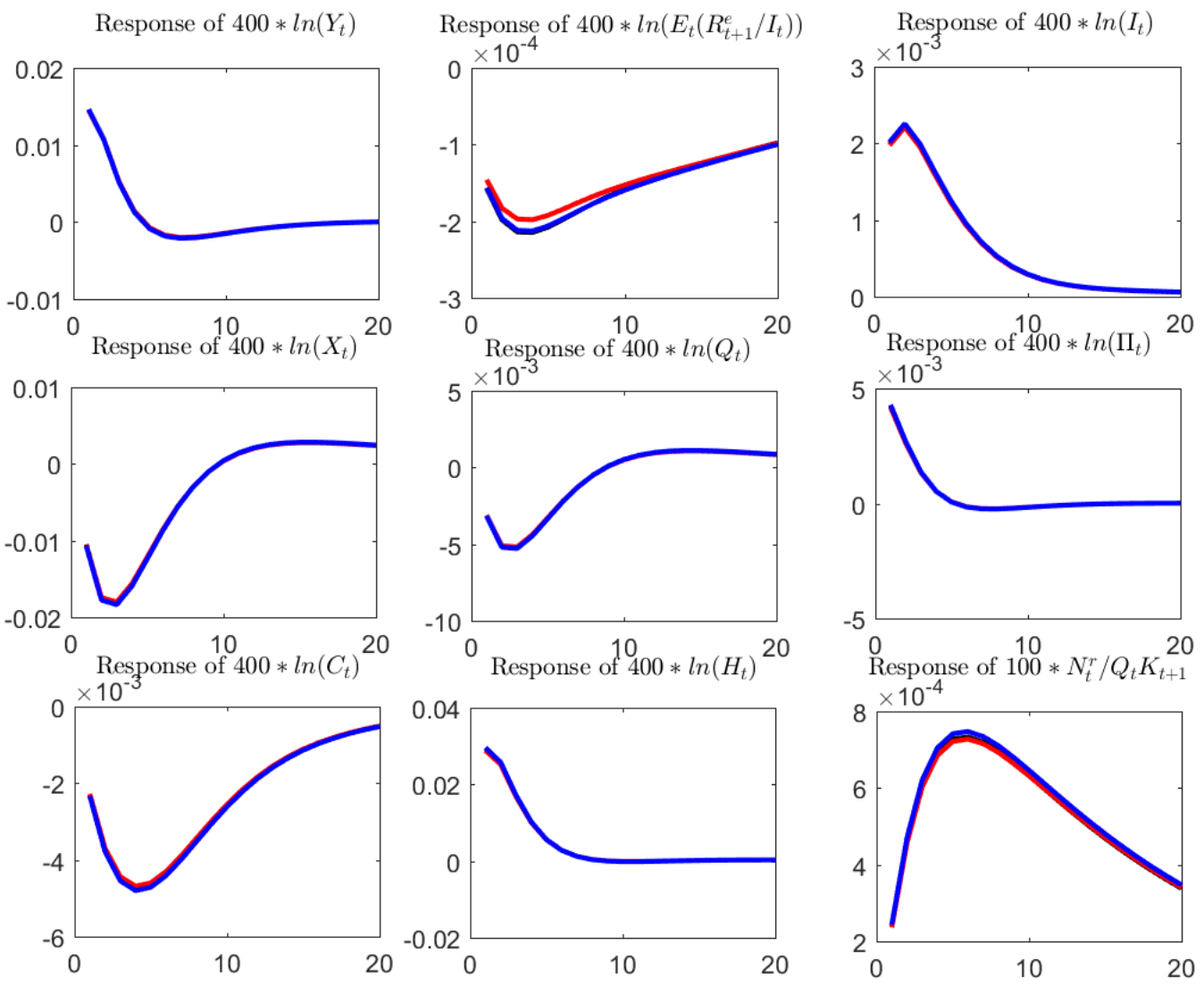

high spread

high interest rate

- high leverage 
Figure 9C. Generalized Impulse Response Functions: Response to a +1 Standard Deviation Micro-Uncertainty Shock, Conditional on High-Values of Spread, Interest Rate, and Leverage Ratio.
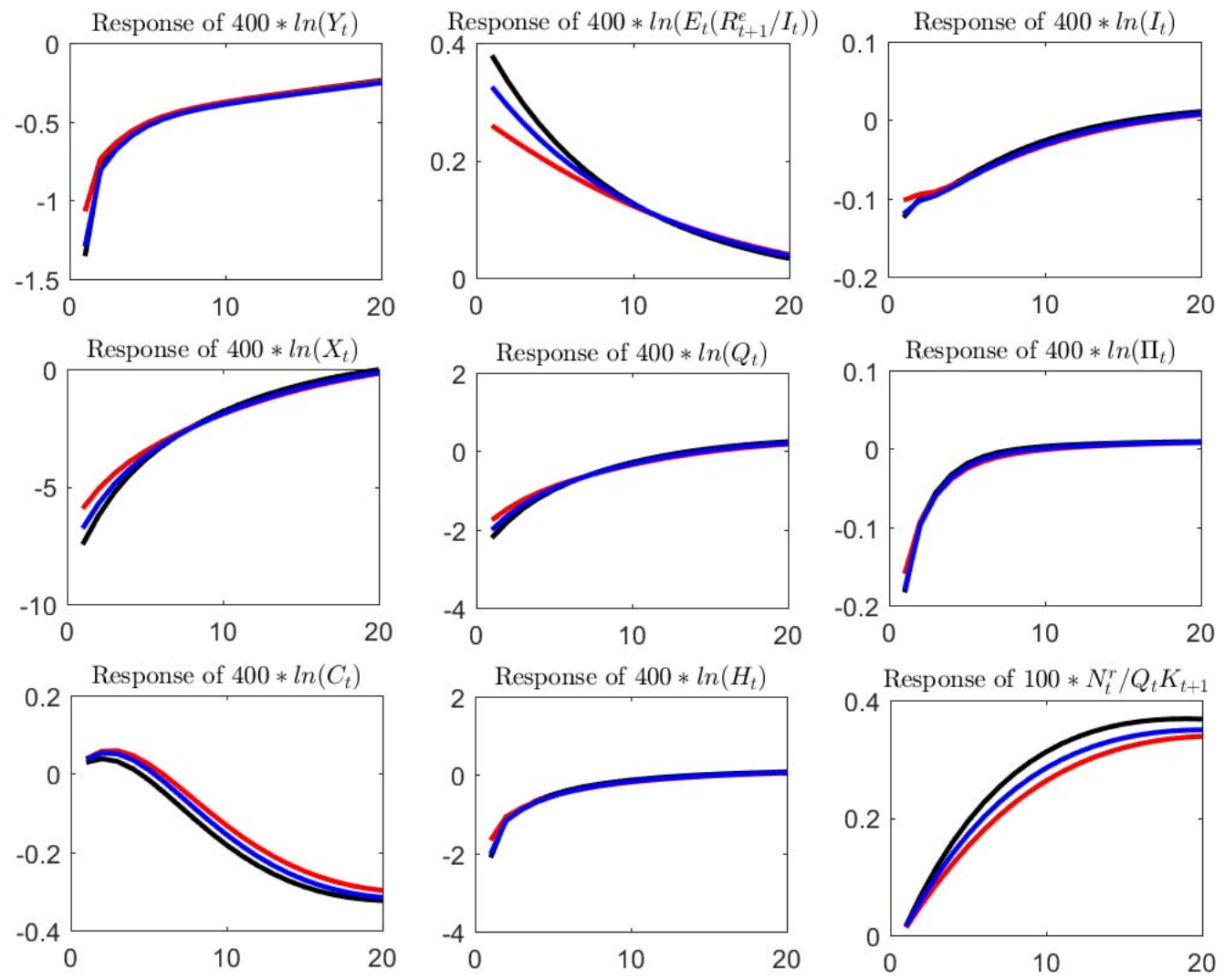

$\longrightarrow$ high spread $\longrightarrow$ high interest rate $\longrightarrow$ high leverage 
Figure 9D. Generalized Impulse Response Functions: Response to a +1 Standard Deviation Interest Rate Rule Shock, Conditional on High-Values of Spread, Interest Rate, and Leverage Ratio.
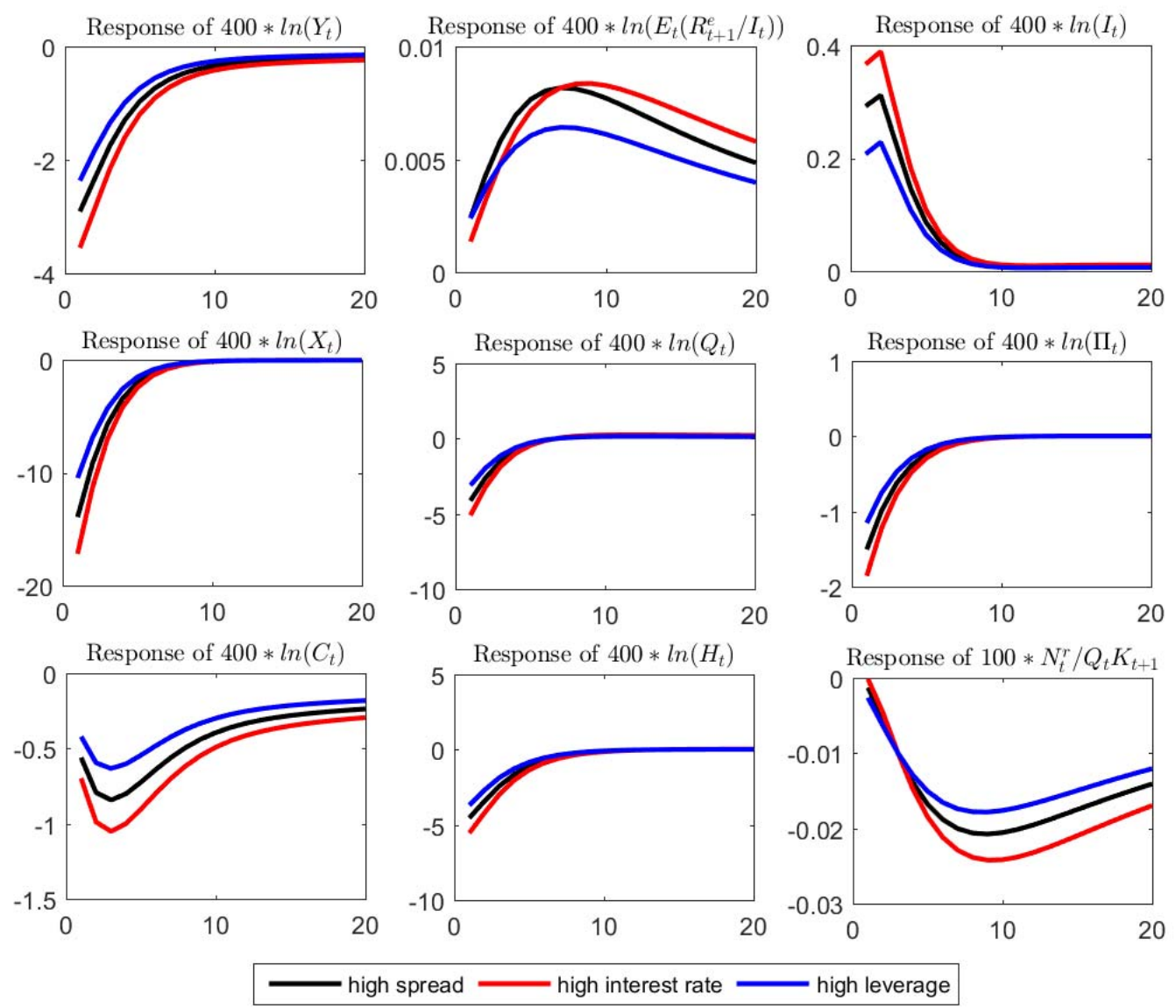
Figure 9E. Generalized Impulse Response Functions: Response to a +1 Standard Deviation Interest Rate Stochastic Volatility Shock, Conditional on High-Values of Spread, Interest Rate, and Leverage Ratio.
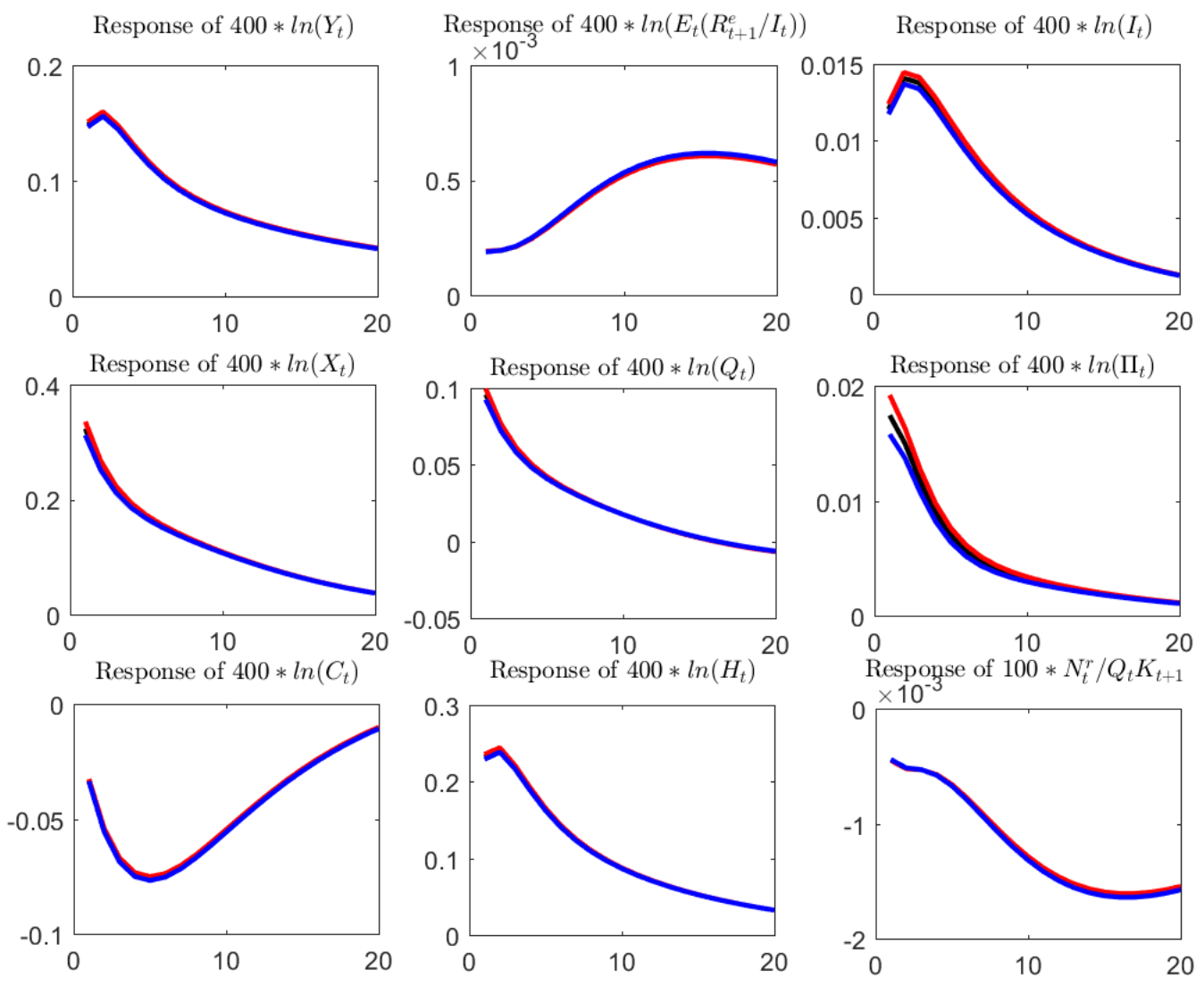

high spread $\longrightarrow$ high interest rate $\longrightarrow$ high leverage 
Figure 10A. Generalized Impulse Response Functions: Response to a +1 Standard Deviation TFP Shock, Conditional on High-Values of Spread, TFP Stochastic Volatility, Interest Rate Stochastic Volatility, and Micro-Uncertainty.
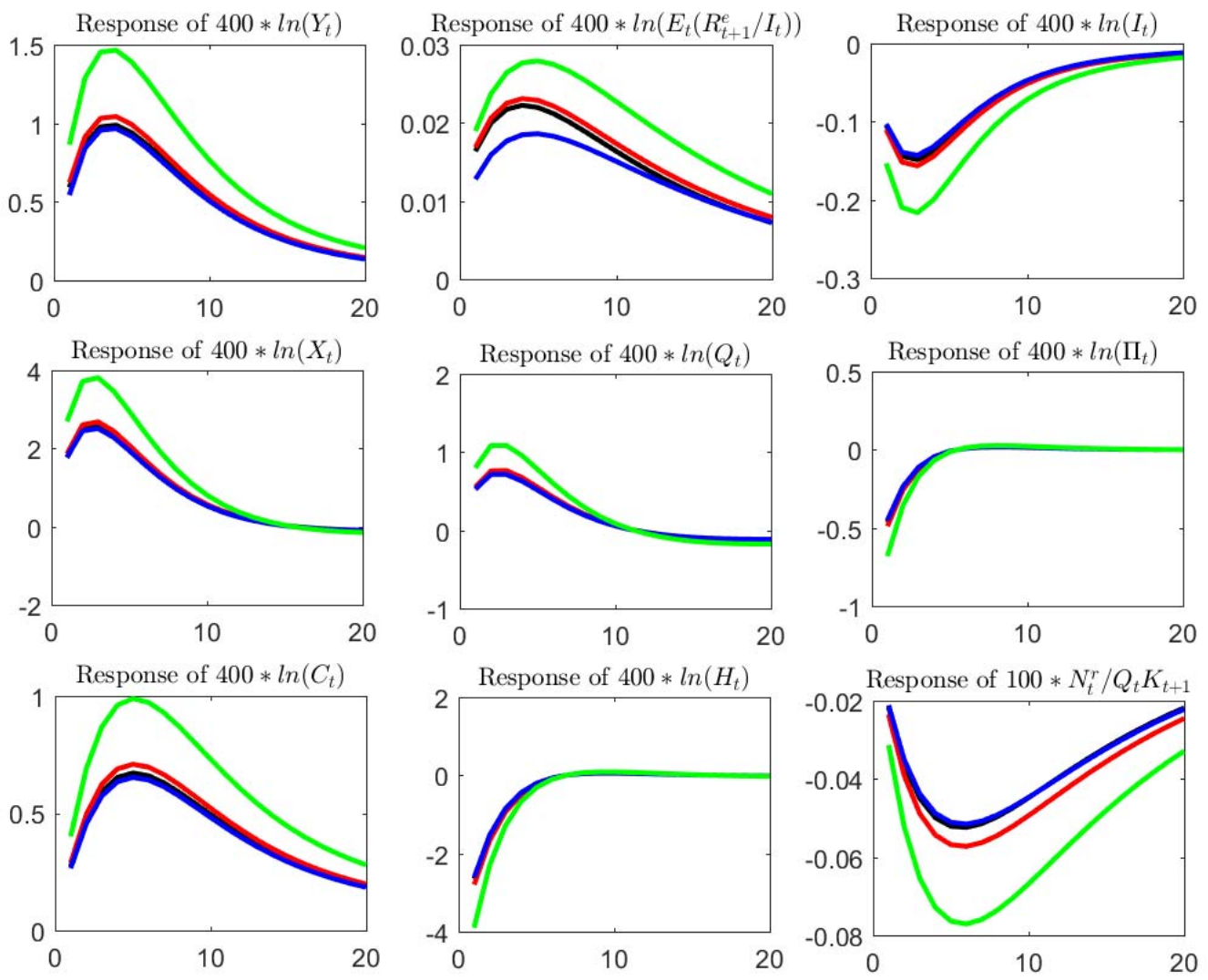

high spread $\longrightarrow$ high micro-uncertainty

high policy uncertainty

high TFP uncertainty 
Figure 10B. Generalized Impulse Response Functions: Response to a +1 Standard Deviation TFP Stochastic Volatility Shock, Conditional on High-Values of Spread, TFP Stochastic Volatility, Interest Rate Stochastic Volatility, and Micro-Uncertainty.
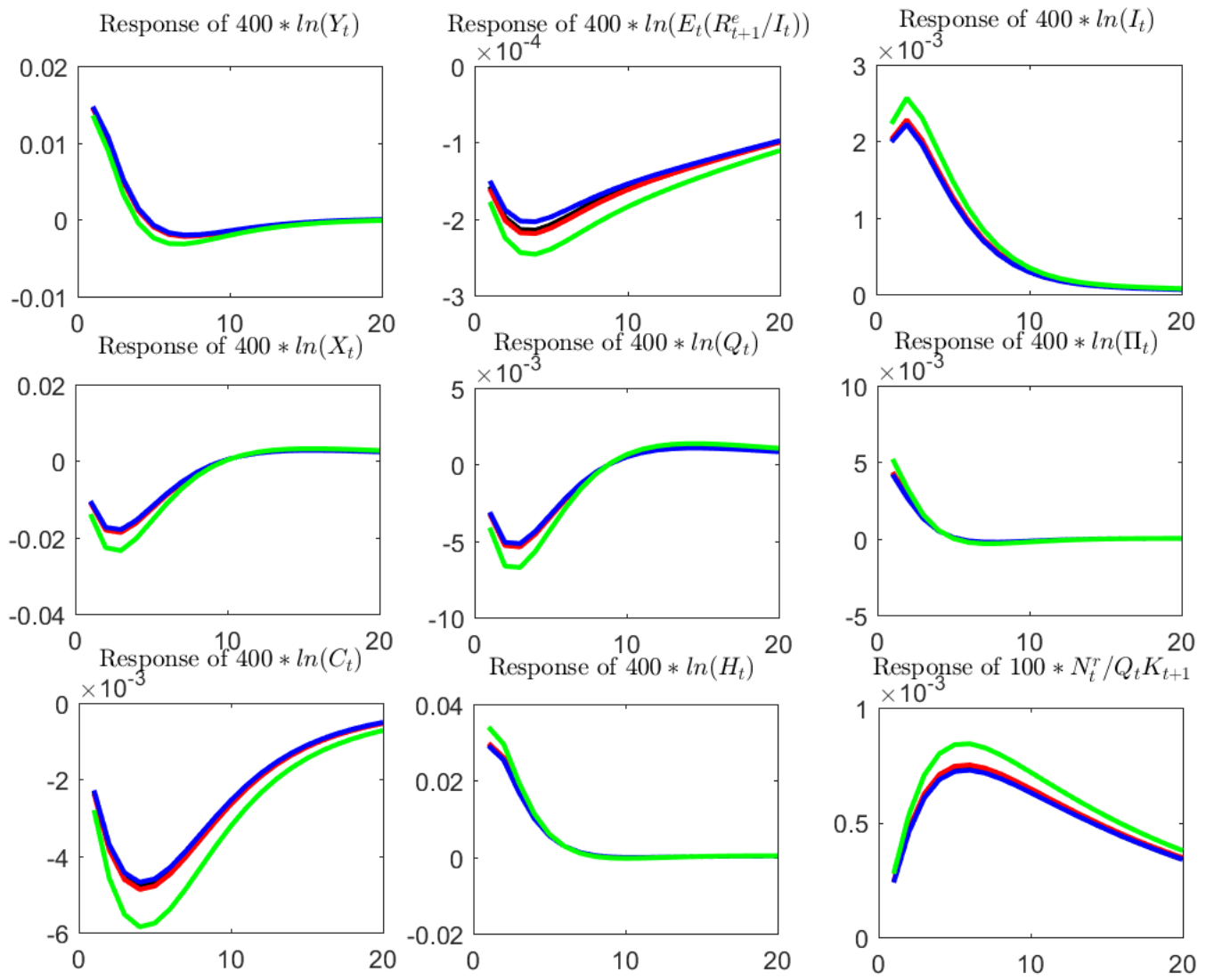

high spread $\longrightarrow$ high micro-uncertainty

high monetary policy uncertainty

high TFP uncertainty 
Figure 10C. Generalized Impulse Response Functions: Response to a +1 Standard Deviation Micro-Uncertainty Shock, Conditional on High-Values of Spread, TFP Stochastic Volatility, Interest Rate Stochastic Volatility, and Micro-Uncertainty.
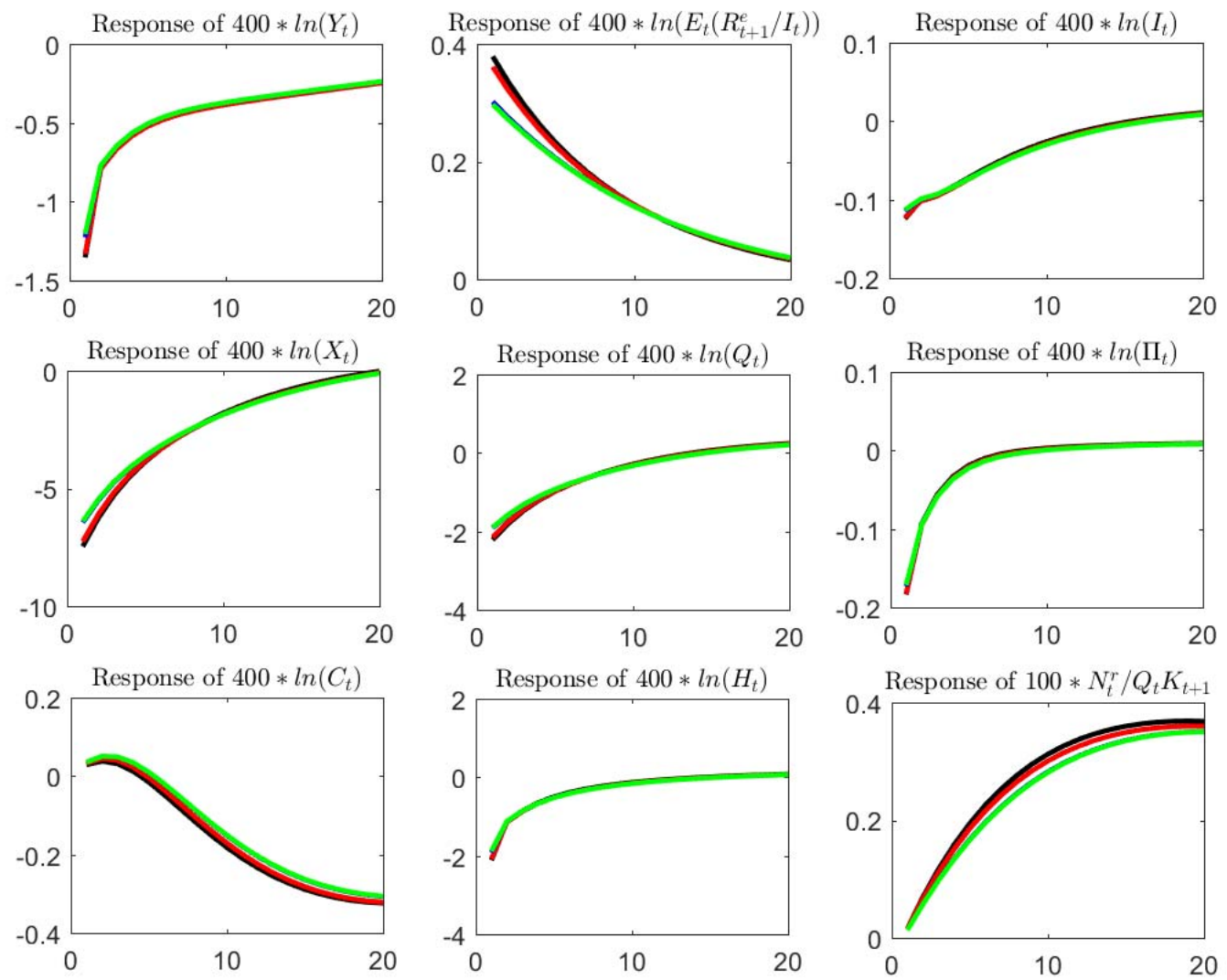

high spread $\longrightarrow$ high micro-uncertainty

high monetary policy uncertainty

- high TFP uncertainty 
Figure 10D. Generalized Impulse Response Functions: Response to a +1 Standard Deviation Interest Rate Rule Shock, Conditional on High-Values of Spread, TFP Stochastic Volatility, Interest Rate Stochastic Volatility, and Micro-Uncertainty.
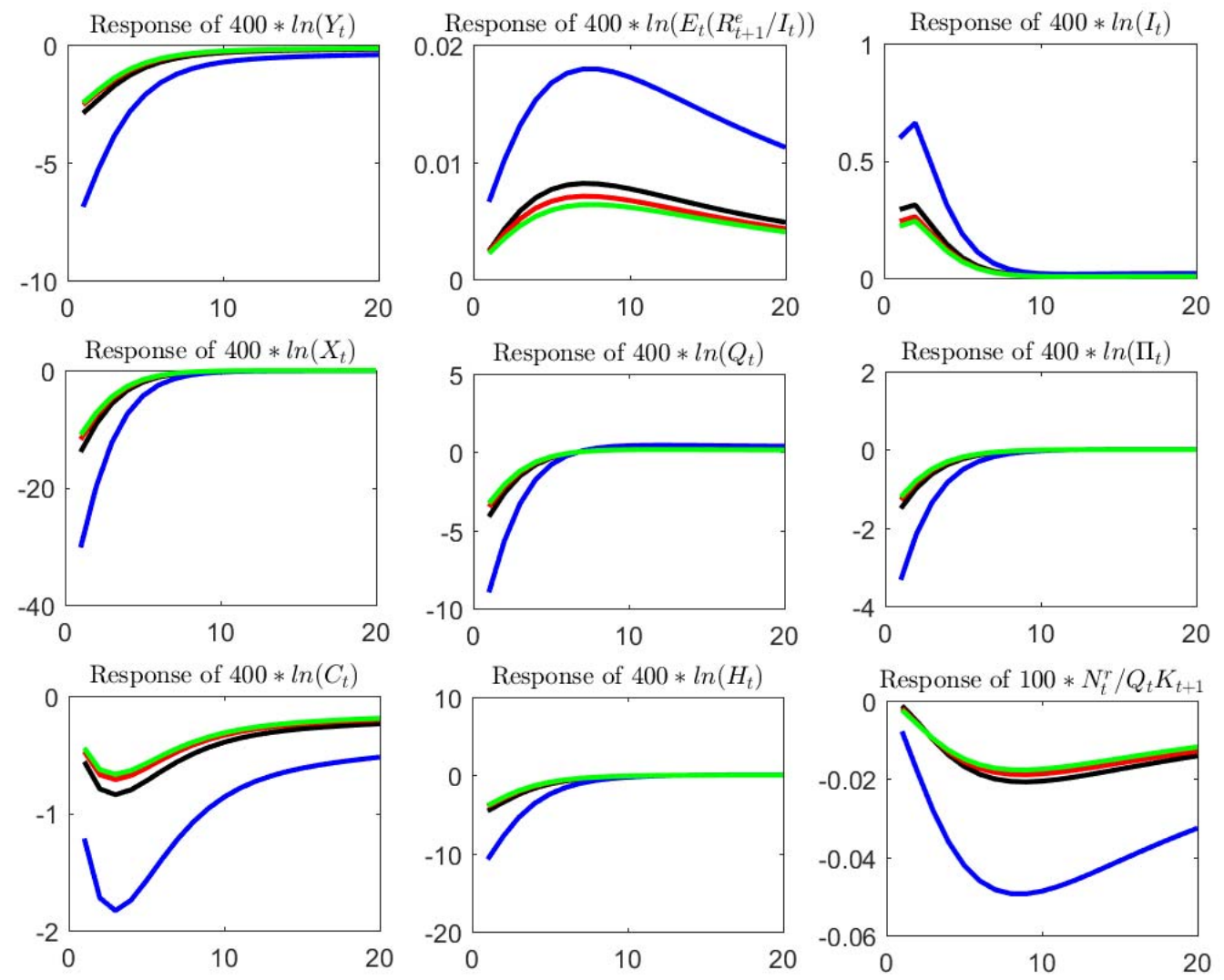

high spread $\longrightarrow$ high micro-uncertainty

high monetary policy uncertainty

- high TFP uncertainty 
Figure 10E. Generalized Impulse Response Functions: Response to a +1 Standard Deviation Interest Rate Stochastic Volatility Shock, Conditional on High-Values of Spread, TFP Stochastic Volatility, Interest Rate Stochastic Volatility, and Micro-Uncertainty.
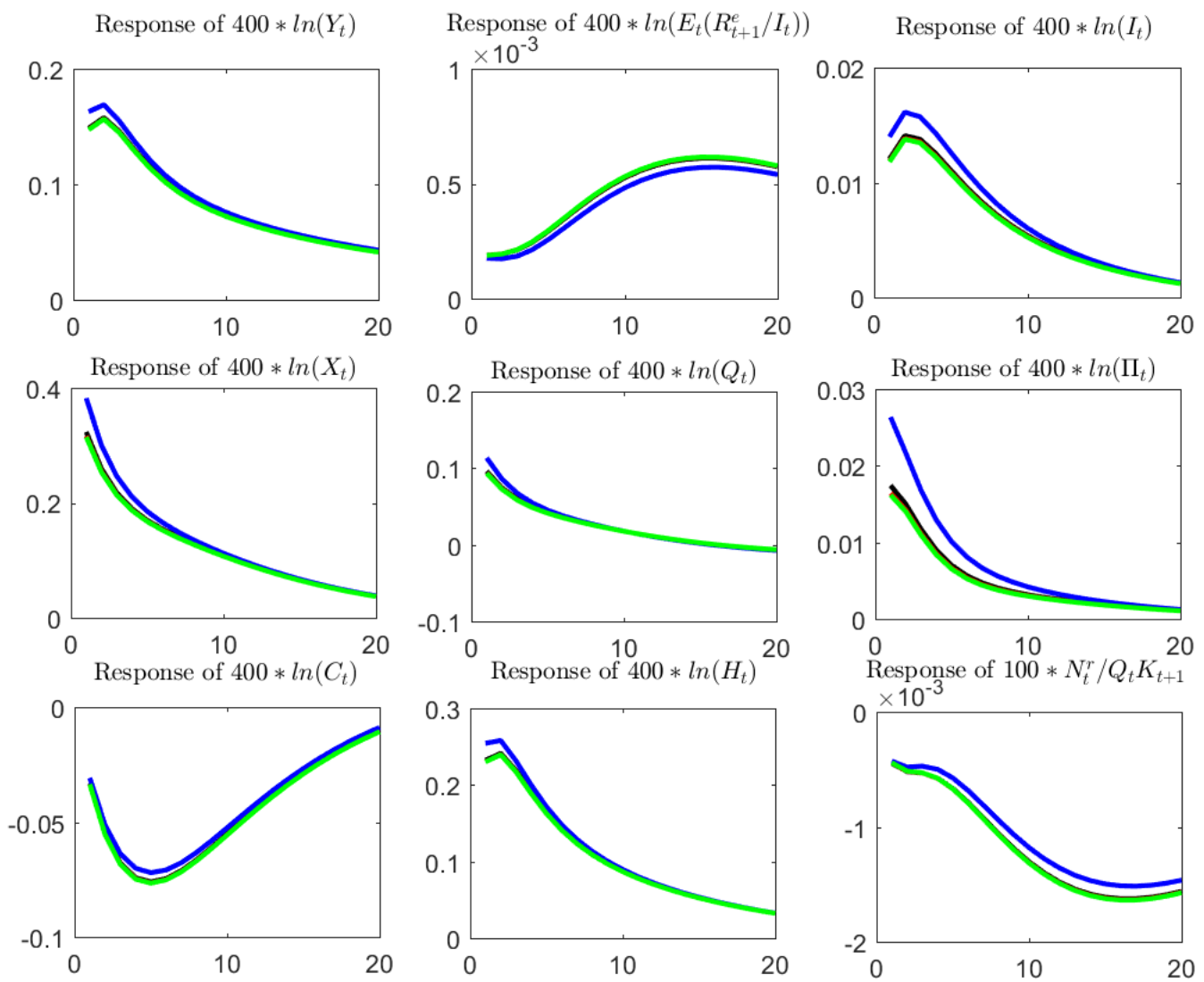

high spread $\longrightarrow$ high micro-uncertainty

high monetary policy uncertainty

high TFP uncertainty 
Figure 11. Probability Density Conditional on the Endogenous Spread: Unconditional, Conditional (5th and 95th percentile of Conditioning Variable). Density Smoothed by Epanechnikov Kernel.
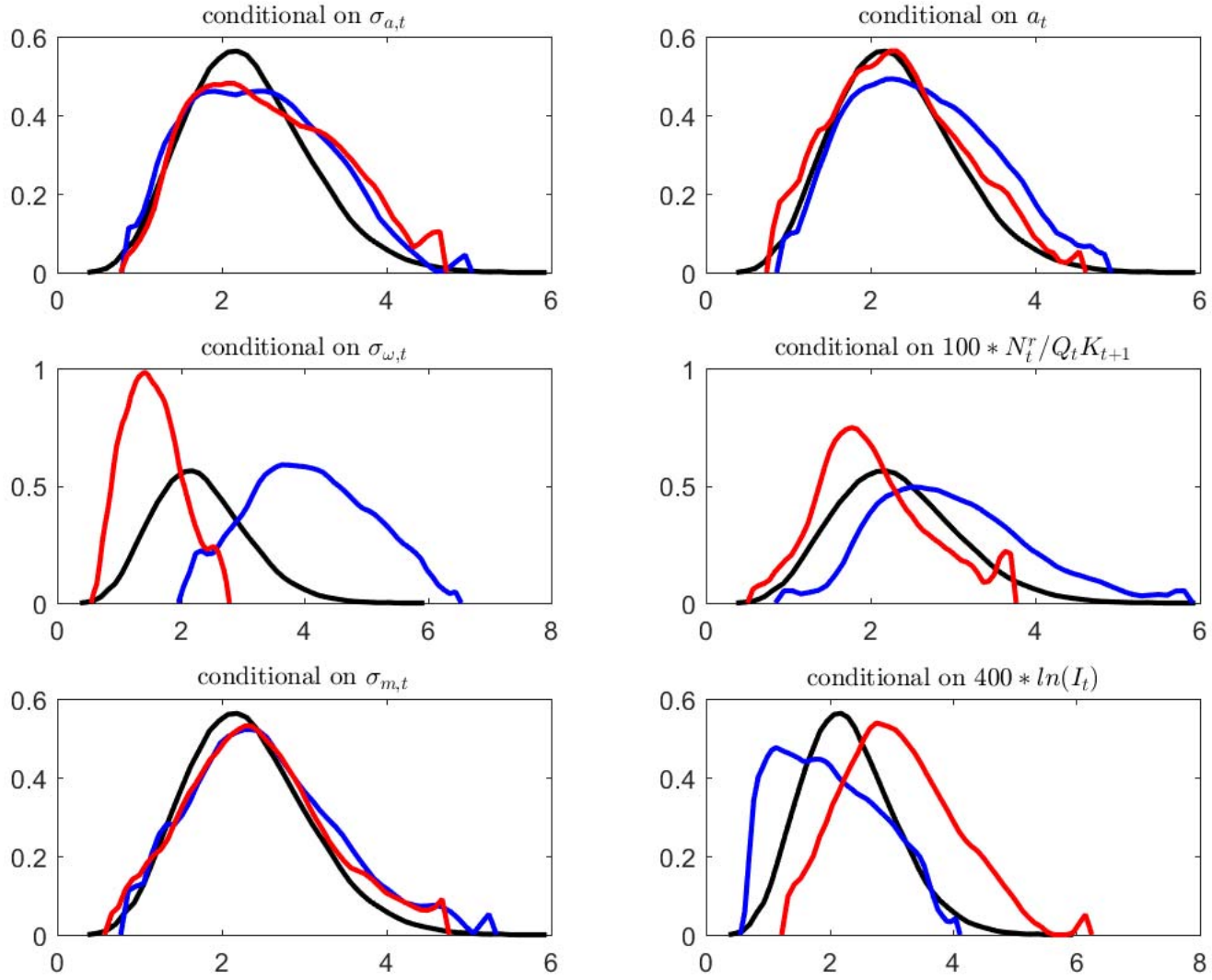

unconditional $\longrightarrow$ conditional-95\% conditional-5\% 
Figure 12A. Joint Distribution of the Endogenous Spread and TFP.

Joint distribution of $400 * \ln \left(E_{t}\left(R_{t+1}^{e} / I_{t}\right)\right)$ and $a_{t}$
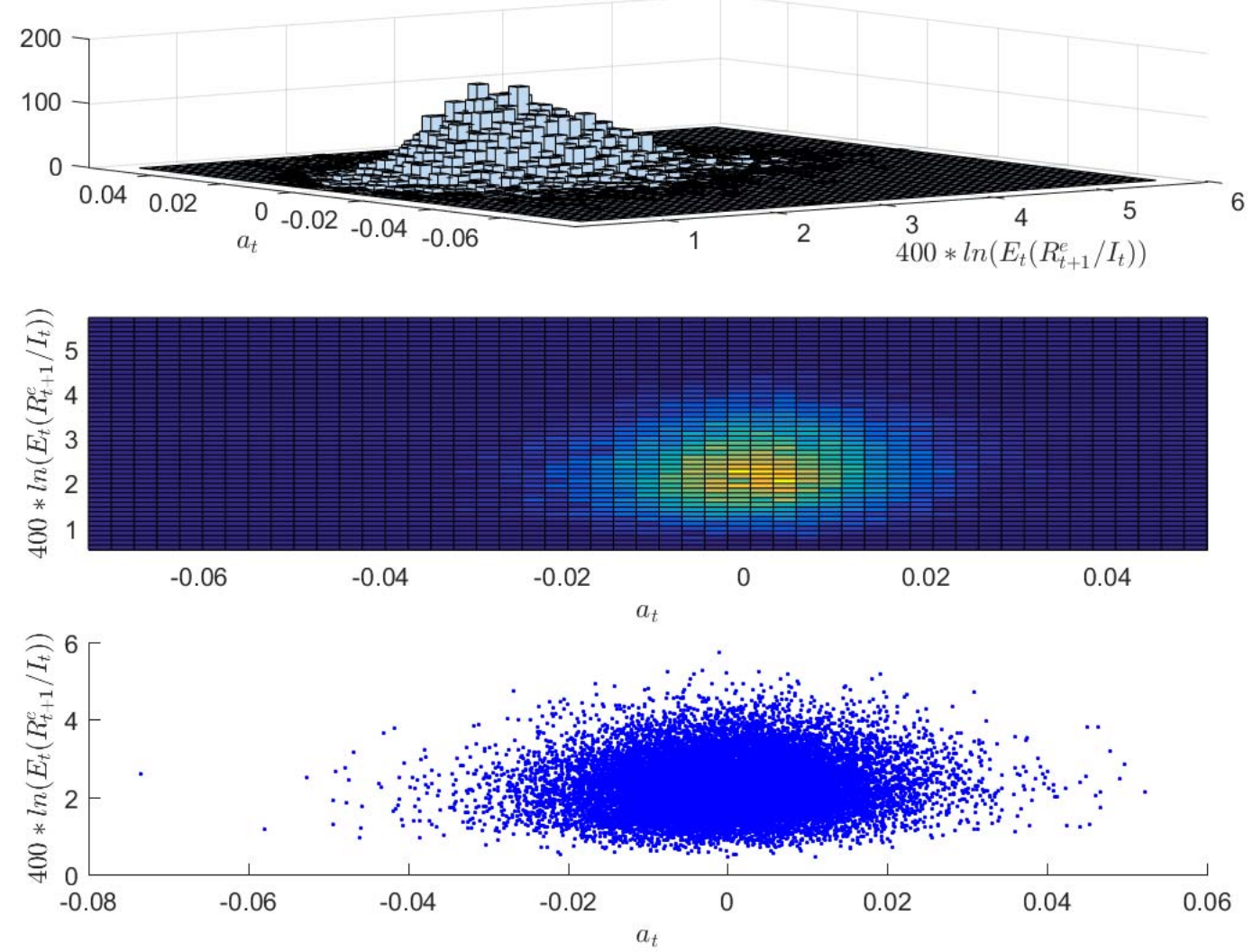
Figure 12B. Joint Distribution of the Endogenous Spread and Interest Rate.

Joint distribution of $400 * \ln \left(E_{t}\left(R_{t+1}^{e} / I_{t}\right)\right)$ and $400 * \ln \left(I_{t}\right)$
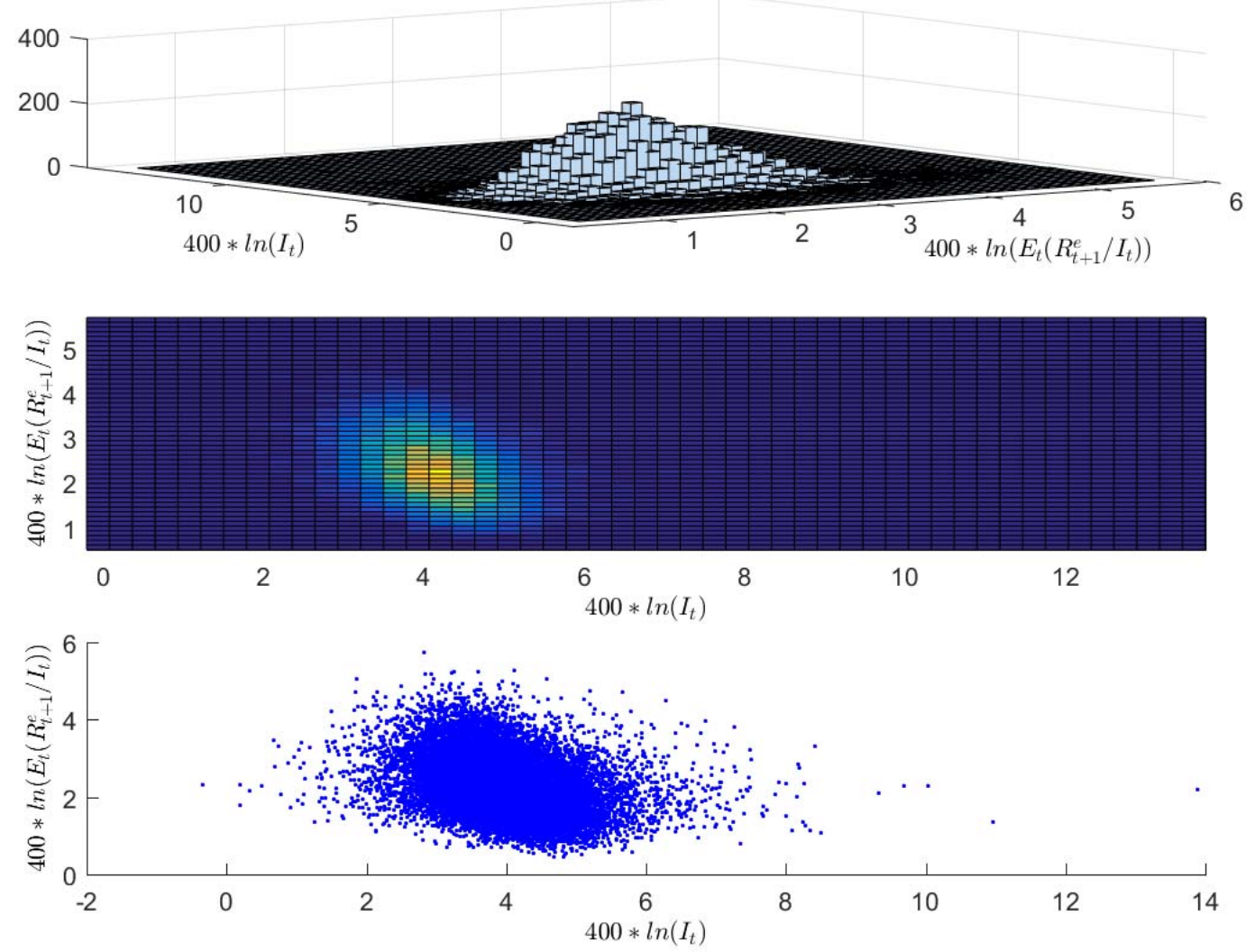
Figure 12C. Joint Distribution of the Endogenous Spread and TFP Stochastic Volatility.
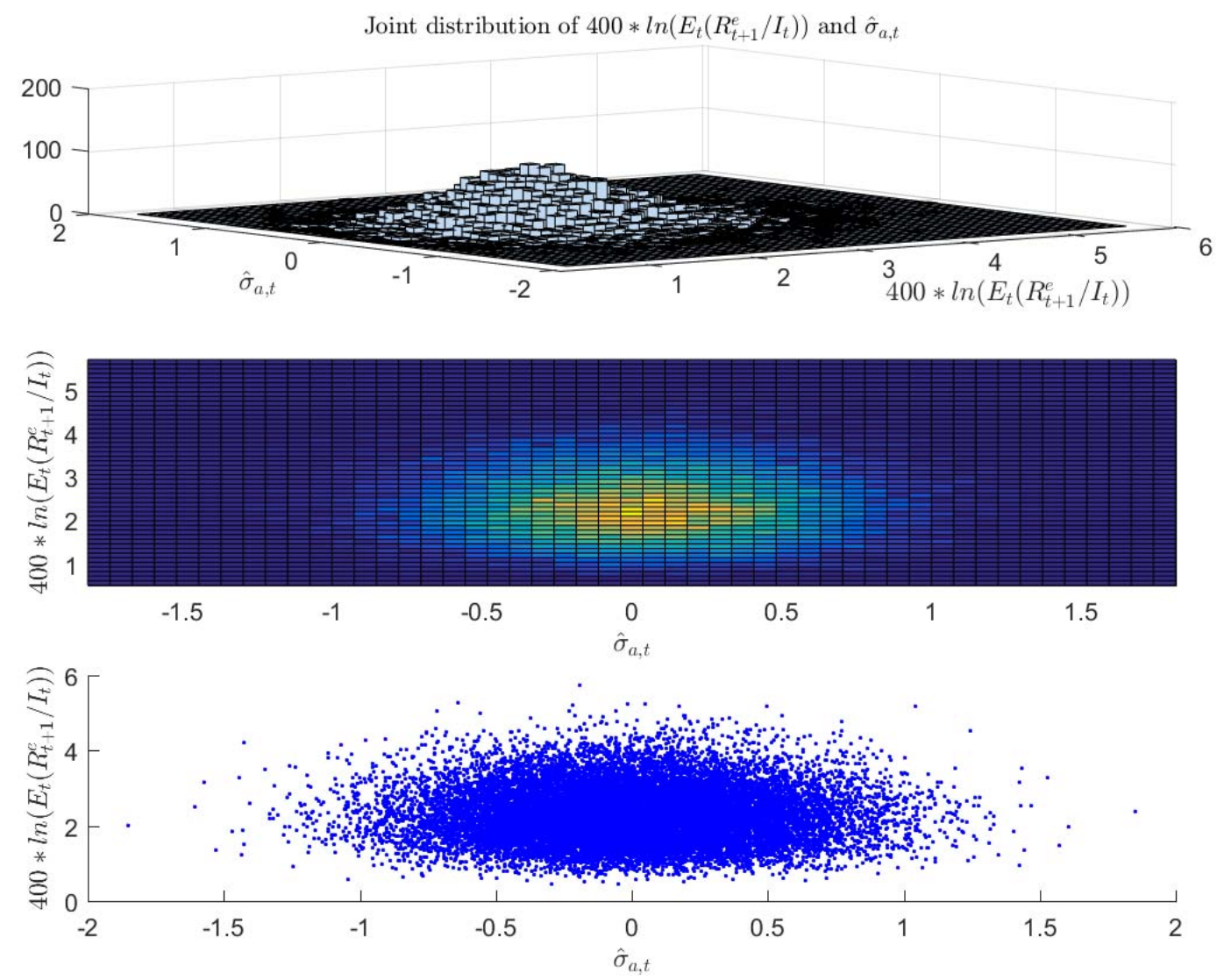
Figure 12D. Joint Distribution of the Endogenous Spread and Interest Rate Stochastic Volatility.

Joint distribution of $400 * \ln \left(E_{t}\left(R_{t+1}^{e} / I_{t}\right)\right)$ and $\hat{\sigma}_{m, t}$
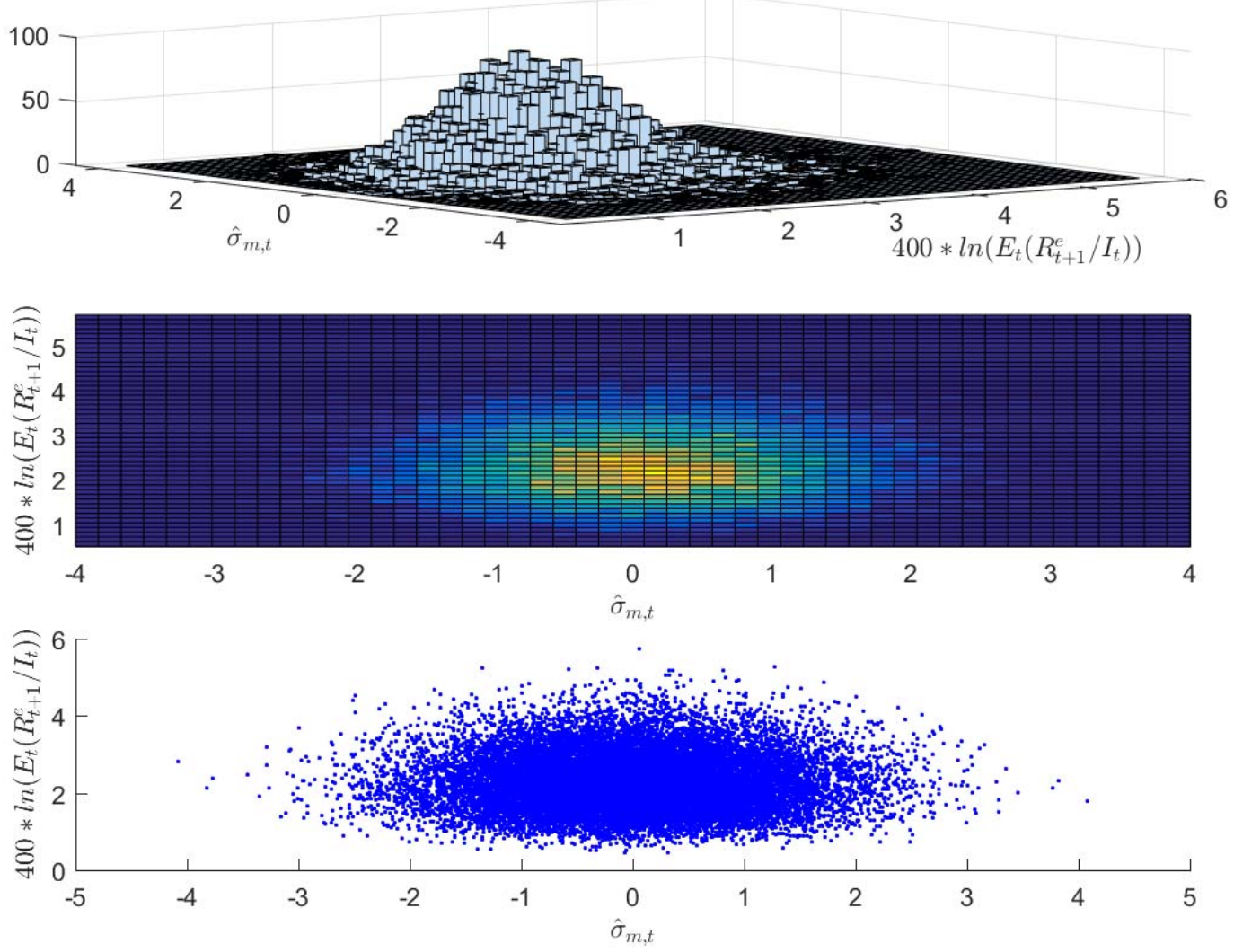
Figure 12E. Joint Distribution of the Endogenous Spread and Micro-Uncertainty.

Joint distribution of $400 * \ln \left(E_{t}\left(R_{t+1}^{e} / I_{t}\right)\right)$ and $\hat{\sigma}_{\omega, t}$
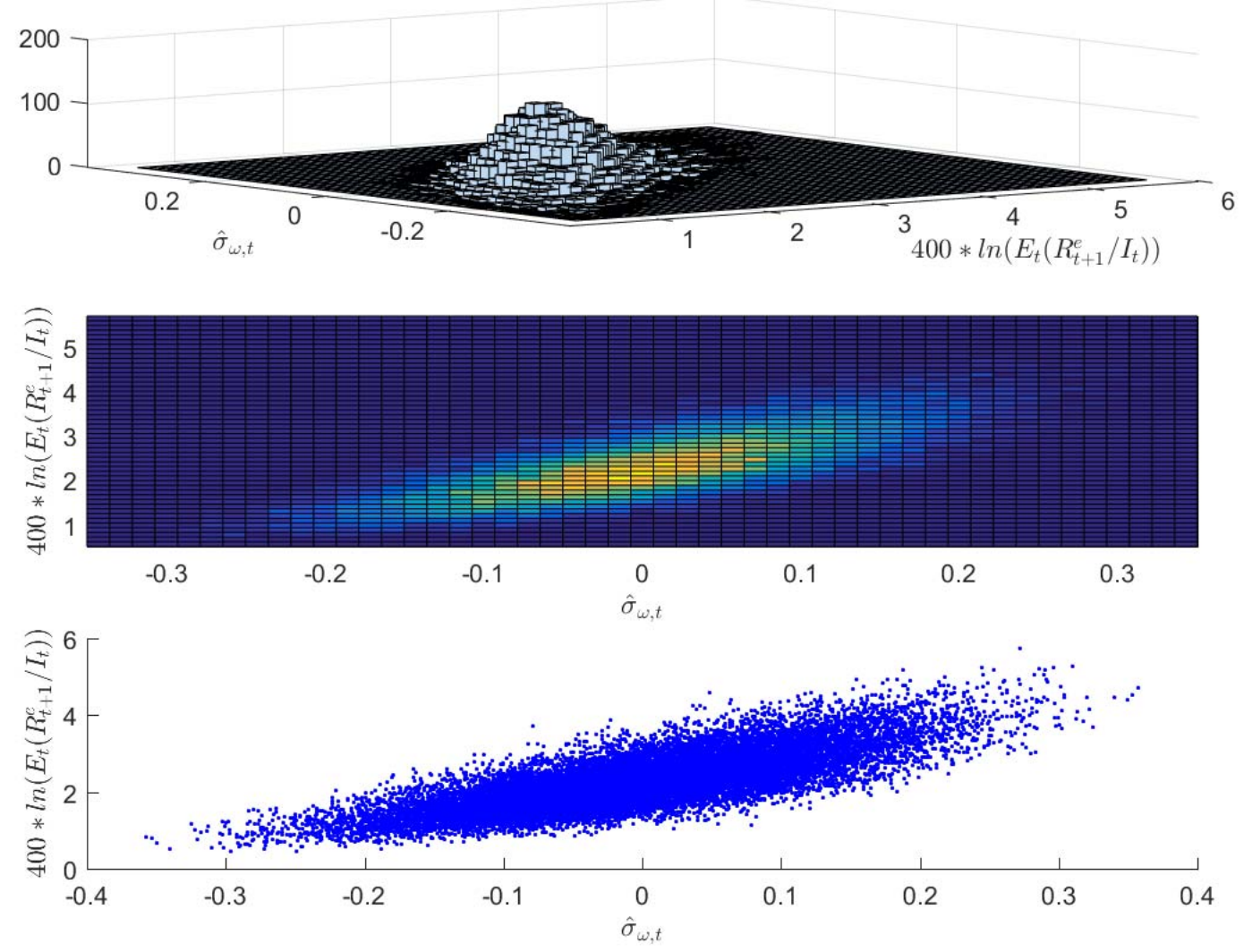
Figure 12F. Joint Distribution of the Endogenous Spread and Capital.

Joint distribution of $400 * \ln \left(E_{t}\left(R_{t+1}^{e} / I_{t}\right)\right)$ and $400 * \ln \left(K_{t+1}\right)$
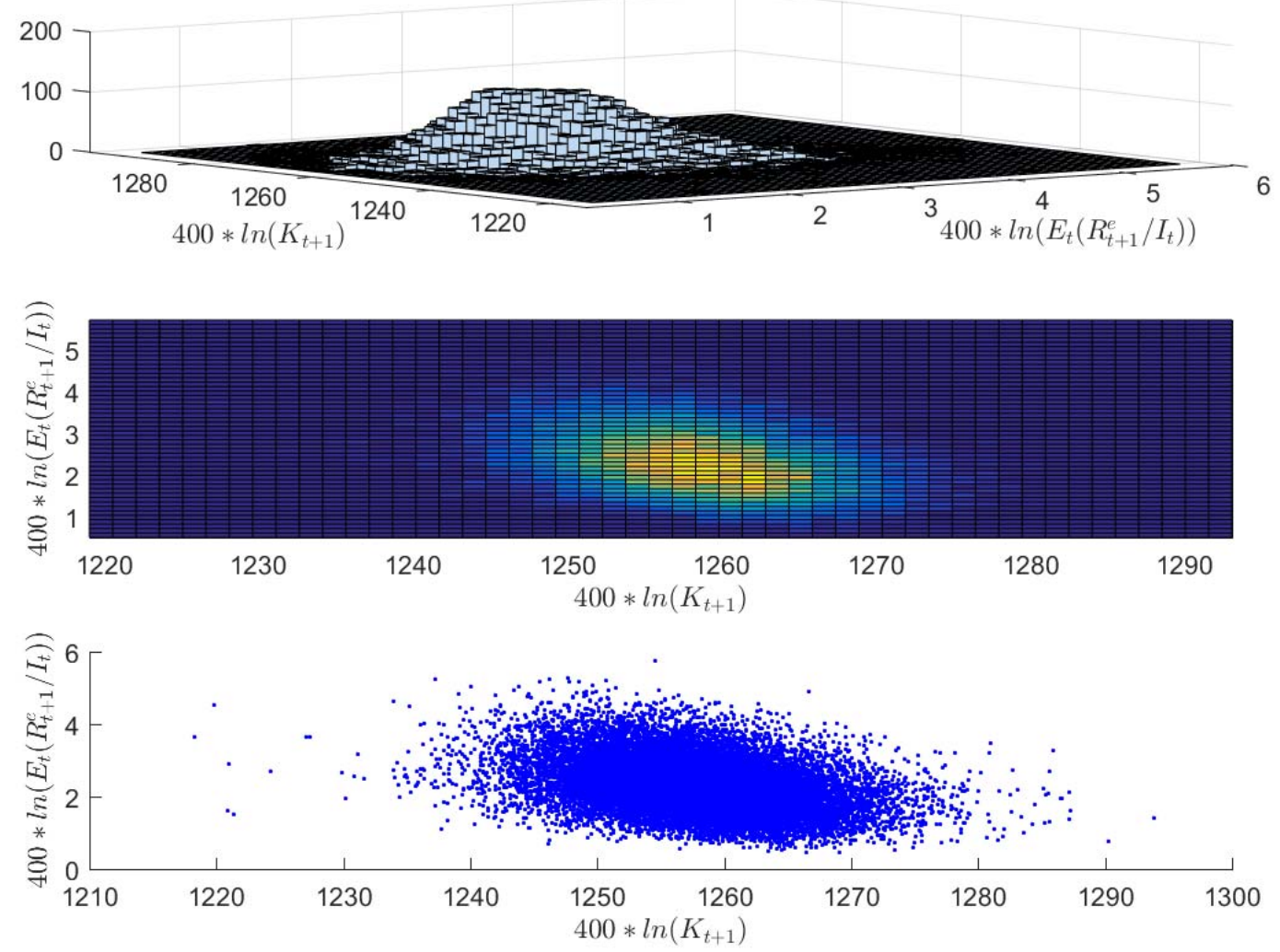
Figure 12G. Joint Distribution of the Endogenous Spread and Real Net Worth.

Joint distribution of $400 * \ln \left(E_{t}\left(R_{t+1}^{e} / I_{t}\right)\right)$ and $400 * \ln \left(N_{t}^{r}\right)$
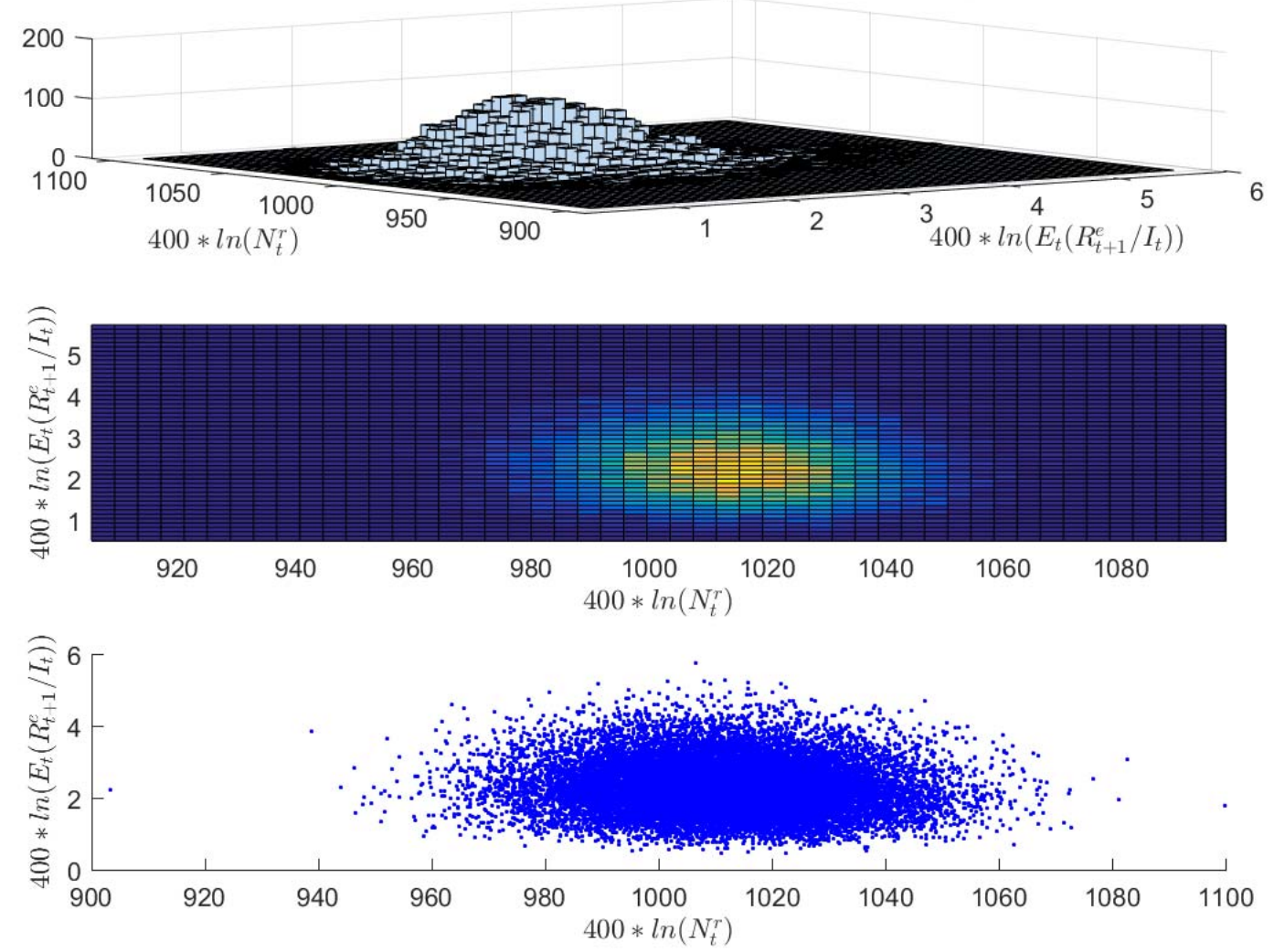
Figure 12H. Joint Distribution of the Endogenous Spread and Output.

Joint distribution of $400 * \ln \left(E_{t}\left(R_{t+1}^{e} / I_{t}\right)\right)$ and $400 * \ln \left(Y_{t}\right)$
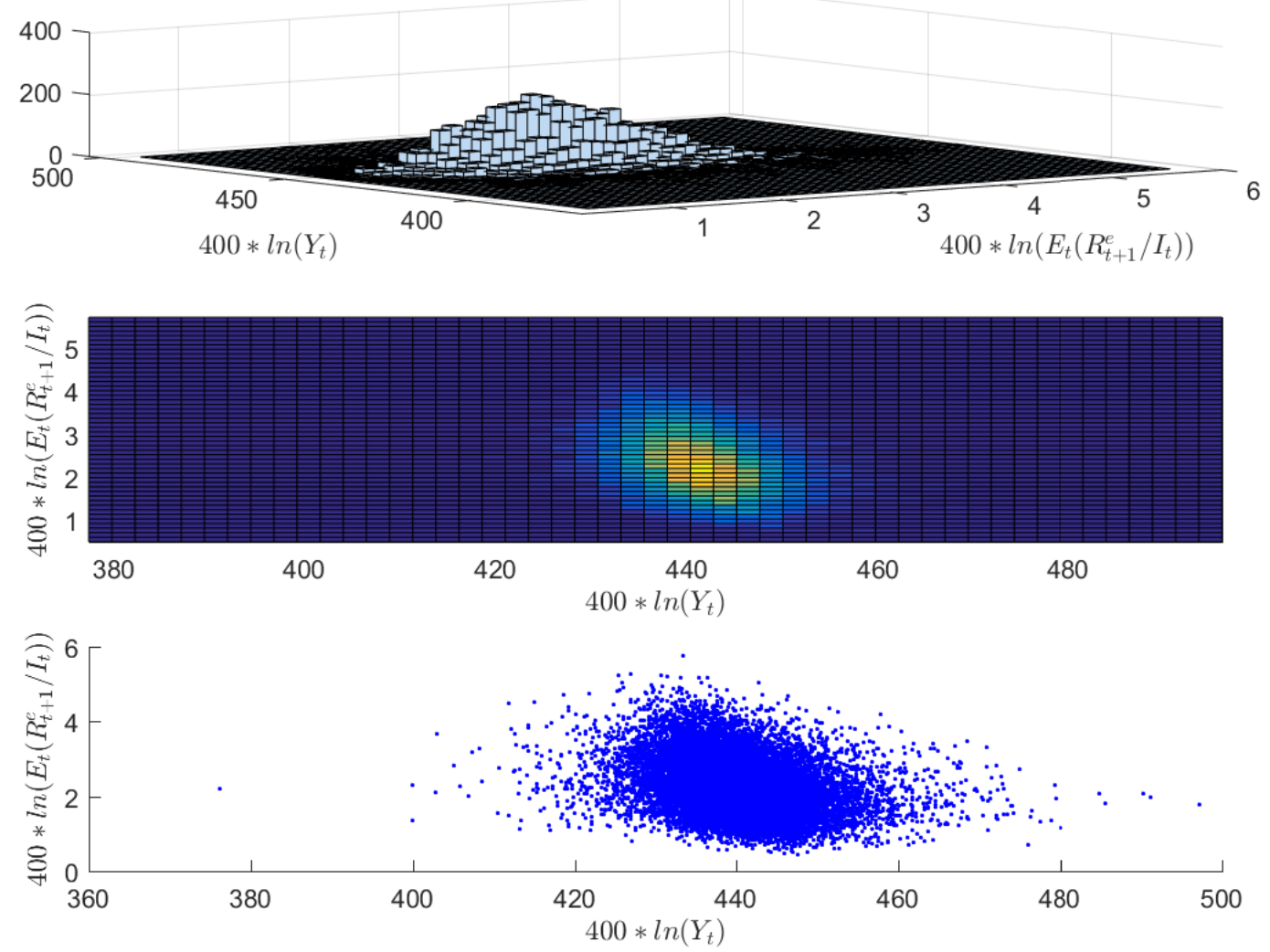
Figure 13A. Joint Distribution of the Endogenous Spread and TFP when Households' Inverse of the Intertemporal Elasticity of Substitution is Increased to $\chi=7$.
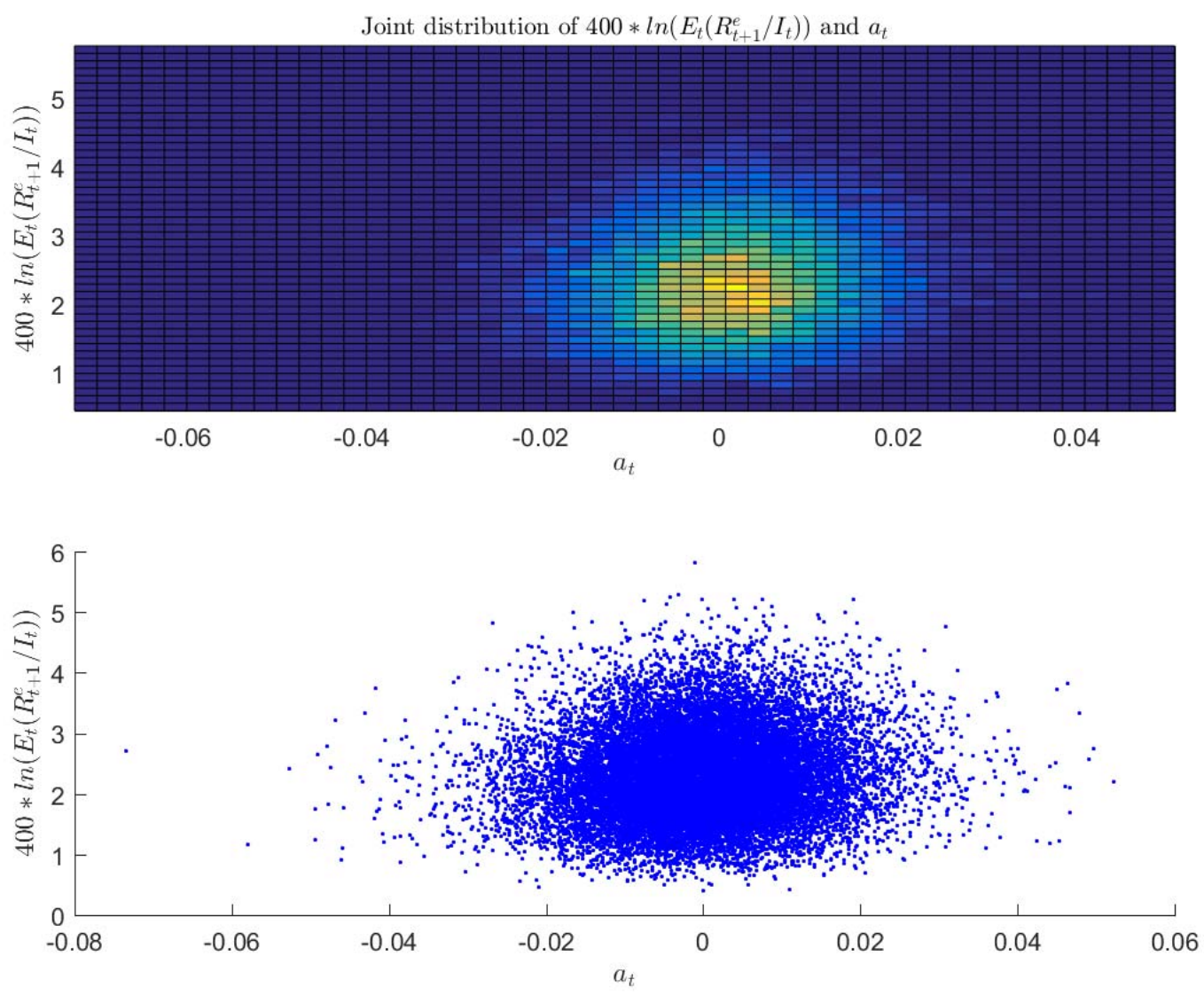
Figure 13B. Joint Distribution of the Endogenous Spread and Interest Rate when Households' Inverse of the Intertemporal Elasticity of Substitution is Increased to $\chi=7$.
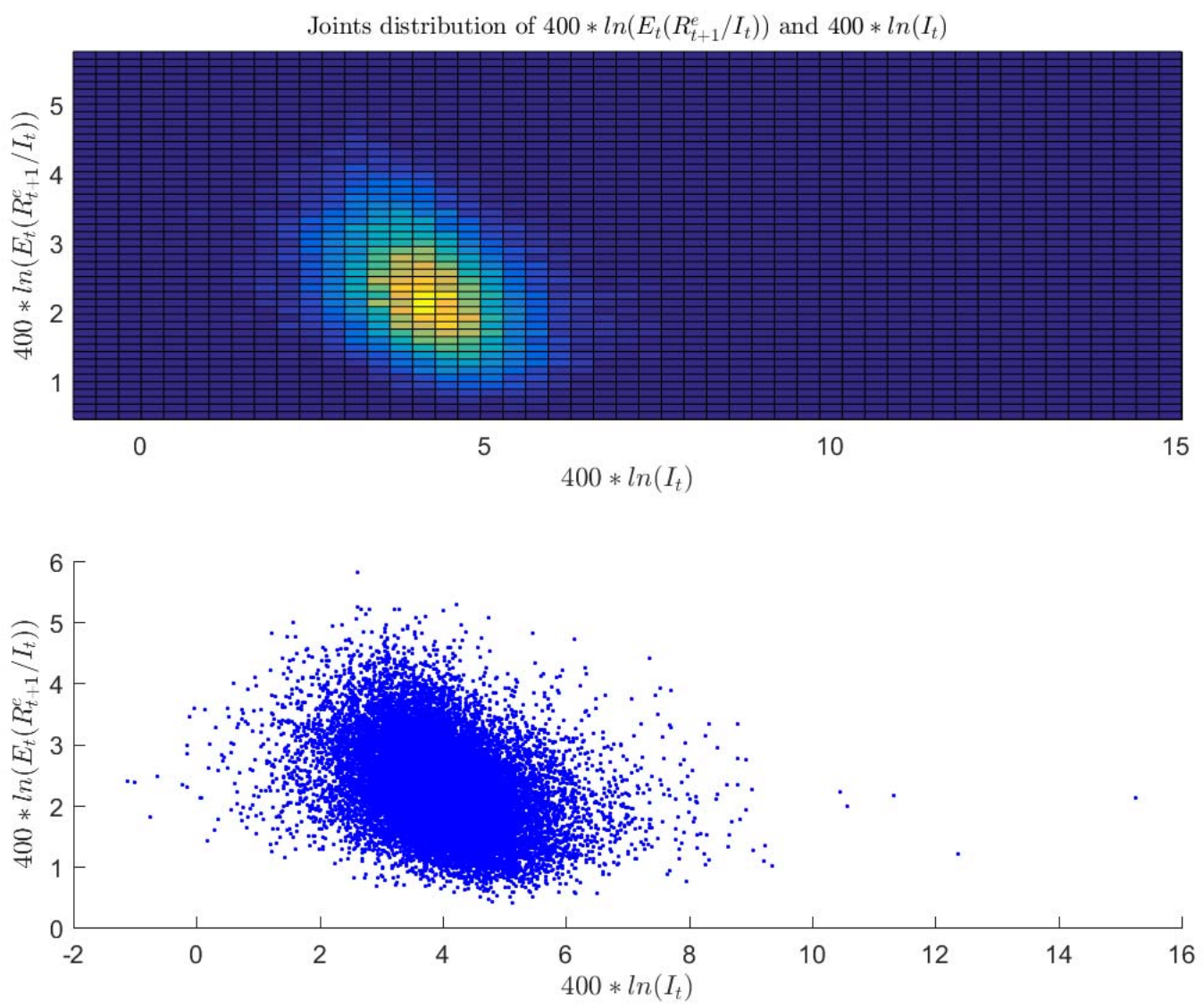
Figure 13C. Joint Distribution of the Endogenous Spread and TFP Stochastic Volatility when Households' Inverse of the Intertemporal Elasticity of Substitution is Increased to $\chi=7$.
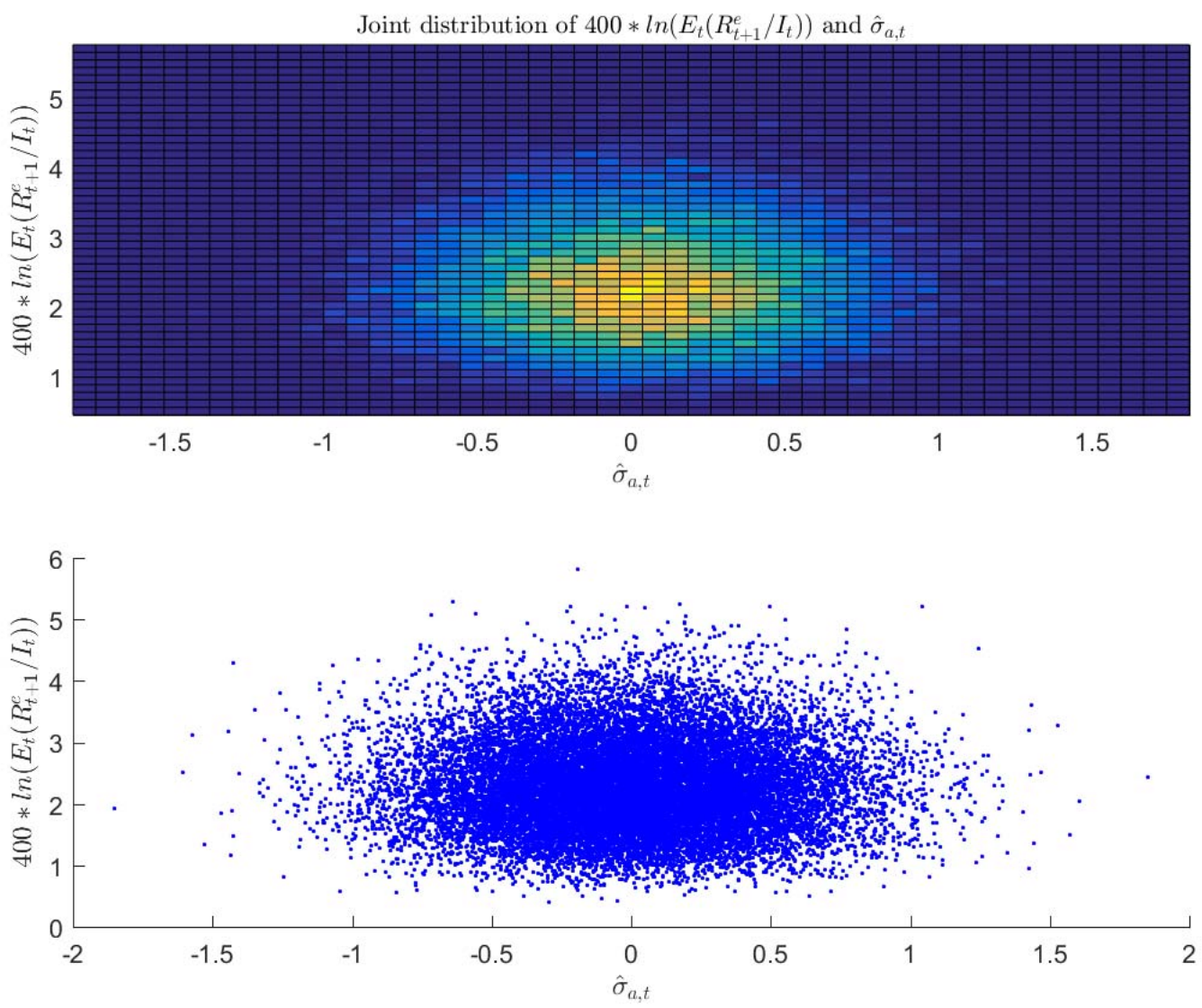
Figure 13D. Joint Distribution of the Endogenous Spread and Interest Rate Stochastic Volatility when Households' Inverse of the Intertemporal Elasticity of Substitution is Increased to $\chi=7$.
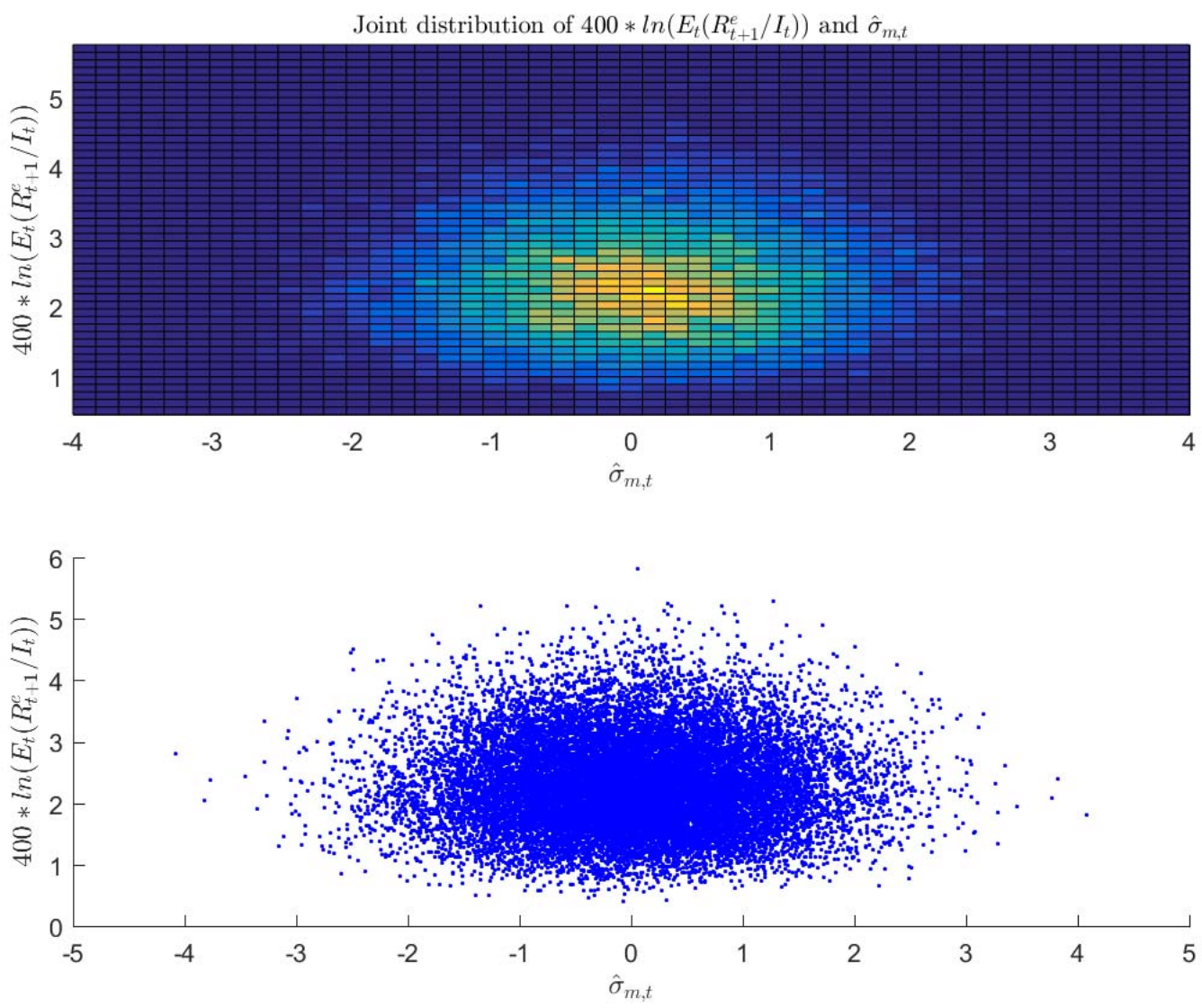
Figure 13E. Joint Distribution of the Endogenous Spread and Micro-Uncertainty when Households' Inverse of the Intertemporal Elasticity of Substitution is Increased to $\chi=7$.
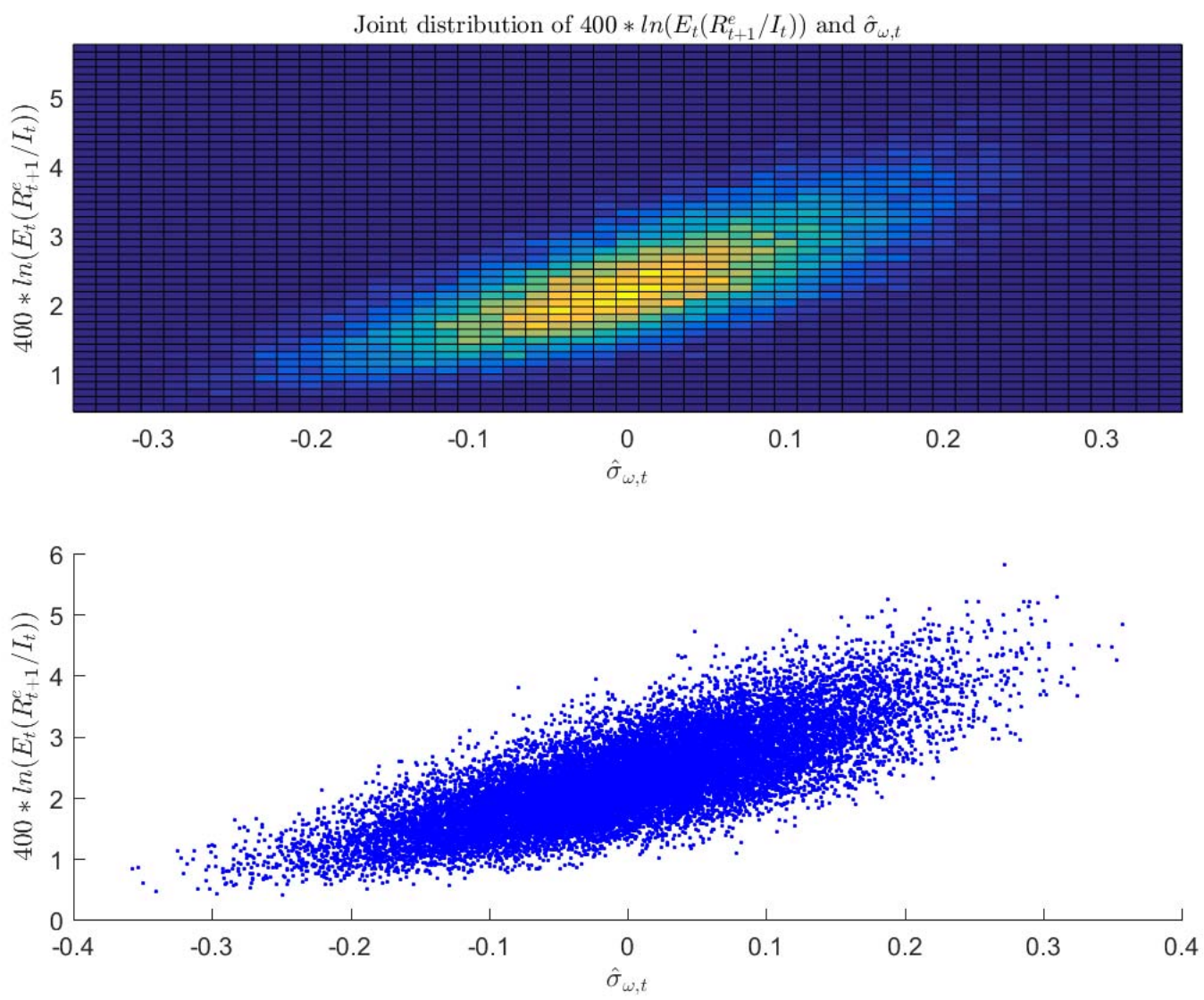
Figure 13F. Joint Distribution of the Endogenous Spread and Capital when Households' Inverse of the Intertemporal Elasticity of Substitution is Increased to $\chi=7$.
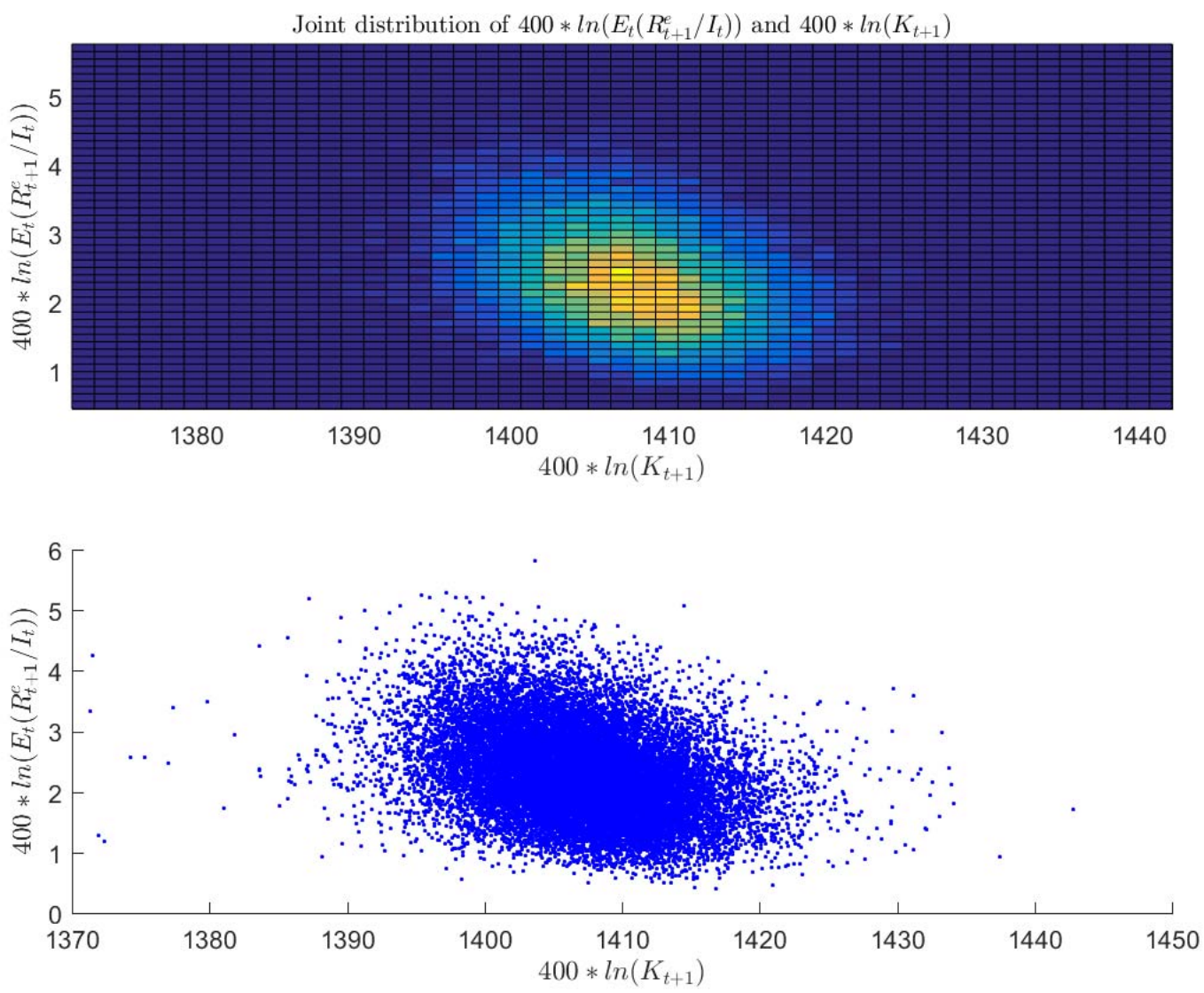
Figure 13G. Joint Distribution of the Endogenous Spread and Real Net Worth when Households' Inverse of the Intertemporal Elasticity of Substitution is Increased to $\chi=7$.
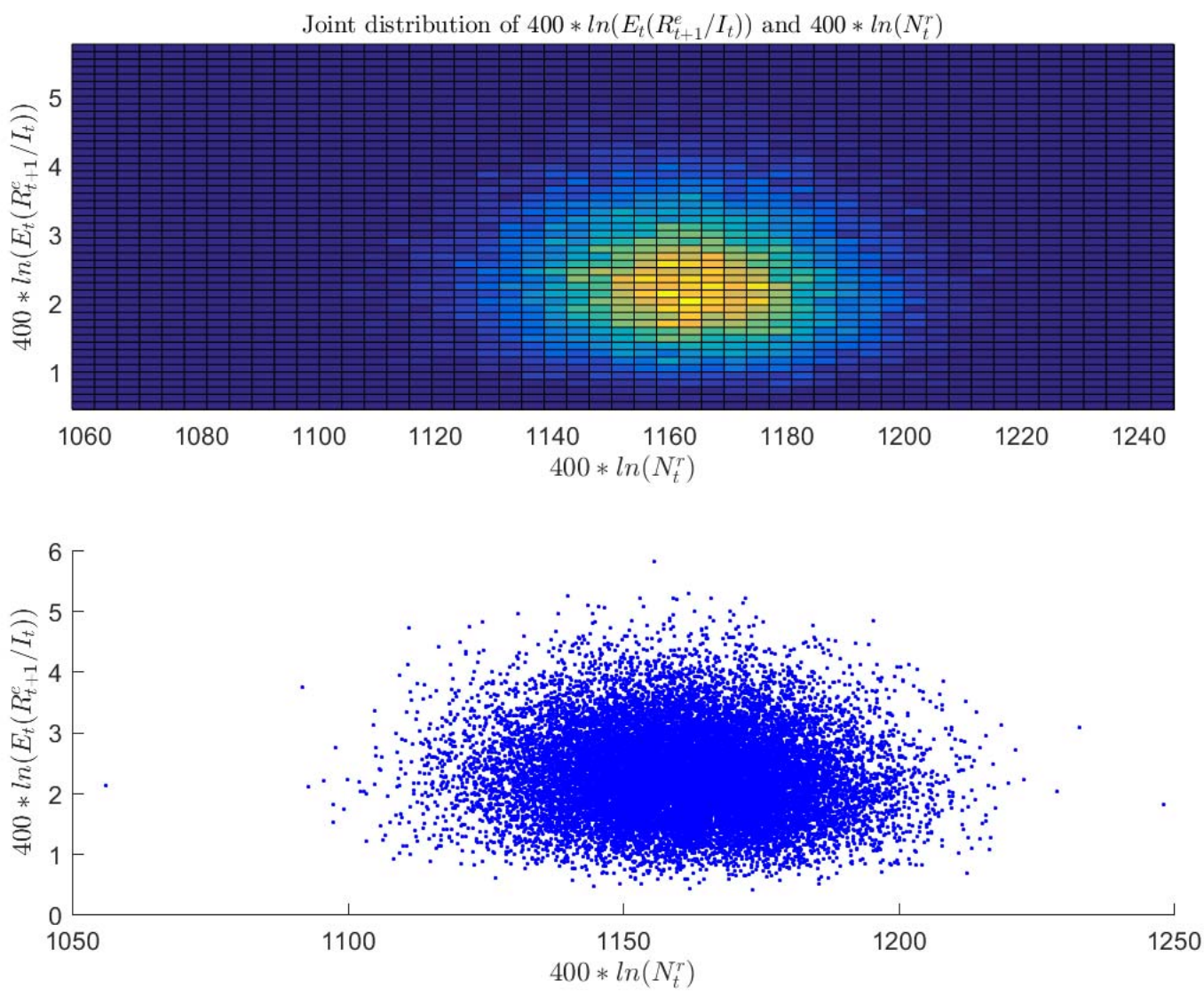
Figure 13H. Joint Distribution of the Endogenous Spread and Output when Households' Inverse of the Intertemporal Elasticity of Substitution is Increased to $\chi=7$.
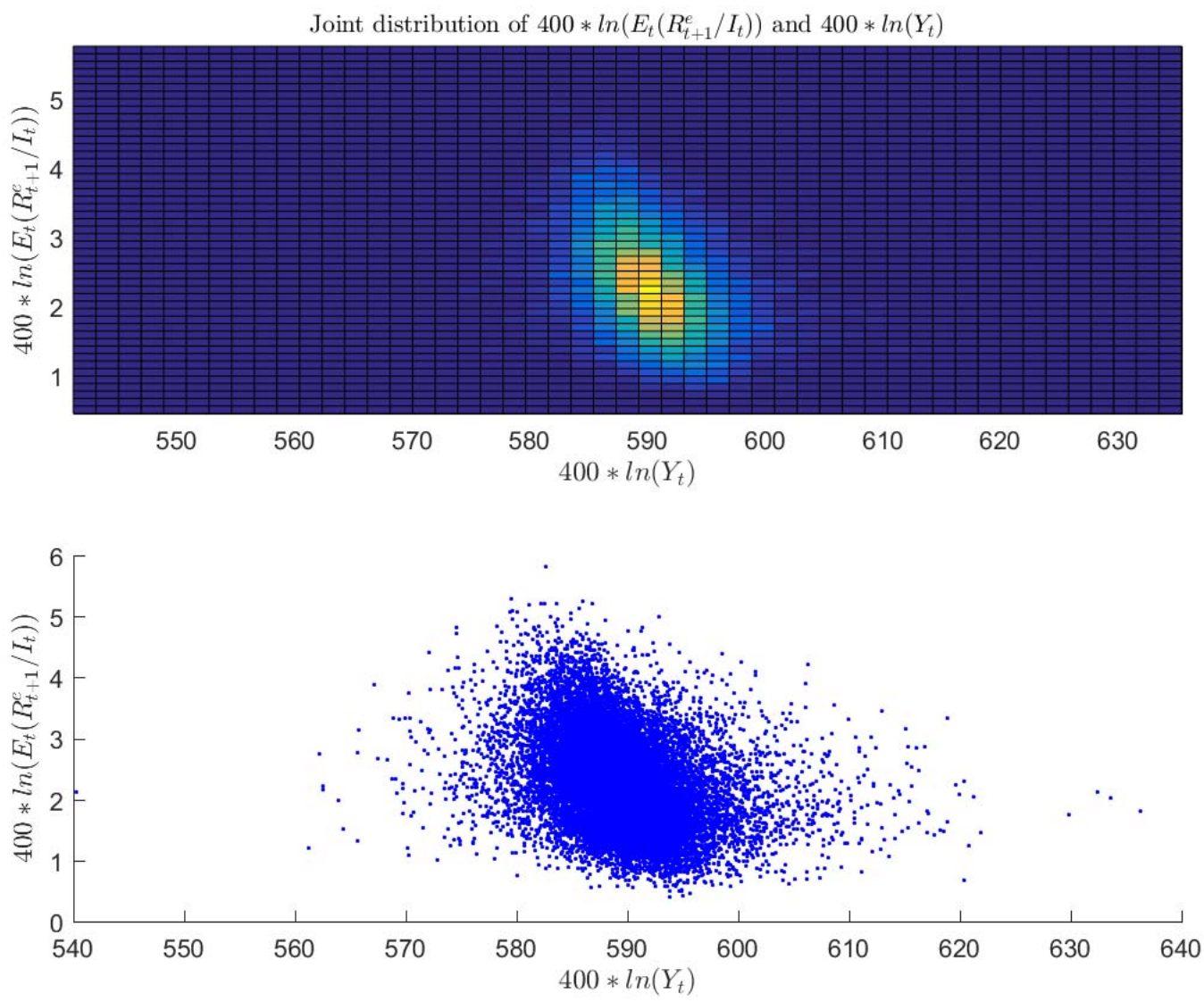


\section{References}

Balke, N., E. Martínez-García, and Z. Zeng (2017). Understanding the Aggregate Effects of Credit Frictions and Uncertainty. Federal Reserve Bank of Dallas Globalization and Monetary Policy Institute Working Paper No. 317.

Basu, S. (1996). Procyclical Productivity: Increasing Returns or Cyclical Utilization? Quarterly Journal of Economics 111(3), 719-51.

Bernanke, B. S., M. L. Gertler, and S. Gilchrist (1999). The Financial Accelerator in a Quantitative Business Cycle Framework. In J. B. Taylor and M. Woodford (Eds.), Handbook of Macroeconomics, vol. 1, Chapter 21, pp. 1341-1393. Elsevier Science B.V.

Born, B. and J. Pfeifer (2014). Policy Risk and the Business Cycle. Journal of Monetary Economics 68(68$85)$.

Christiano, L. J., R. Motto, and M. Rostagno (2014). Risk Shocks. American Economic Review 104(1), $27-65$.

Mehra, Y. (2004). The Output Gap, Expected Future Inflation and Inflation Dynamics: Another Look. The B.E. Journal of Macroeconomics 4 (1), 1-19.

Rotemberg, J. J. (1982). Sticky Prices in the United States. Journal of Political Economy 90(6), 11871211.

Stock, J. H. and M. W. Watson (1999). Forecasting Inflation. Journal of Monetary Economics 44(2), 293-335. 